\title{
VI. APPENDIX CHAPTER II
}

\author{
Abbreviations / Nr. Pages
}

Photographs

Grain shapes according to Tucker

Vickers tests

Vick $1.2-5.2$

$266-268$

\section{Graphics}

Grain size distribution - Graphics

Bulk mineral analysis - Graphics

Clay mineral analysis - Graphics

Simultaneous thermal analysis - Graphics
GSD $1.2-34.2$

BMA $1.2-3.2$

CMA $1.2-4.2$

STA $1.2-2.2$
$269-302$

$303-304$

$304-306$

$306-307$
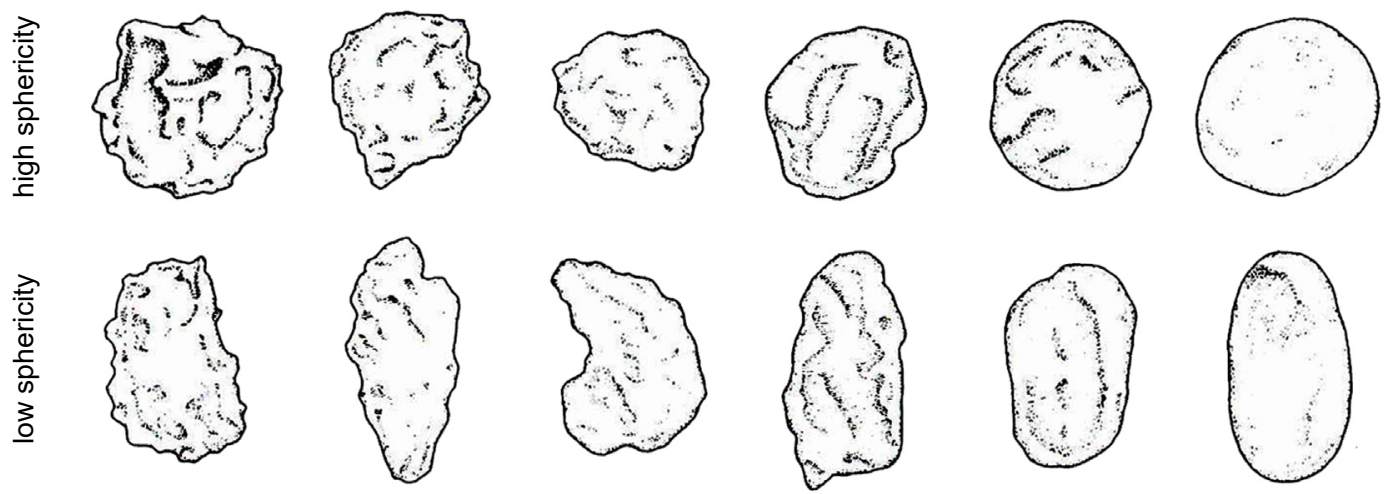

very angular

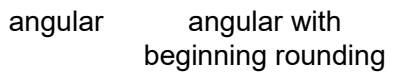

slightly rounded

rounded

well rounded

Fig. 103.2 Grain shapes after Tucker 1985: 18. 

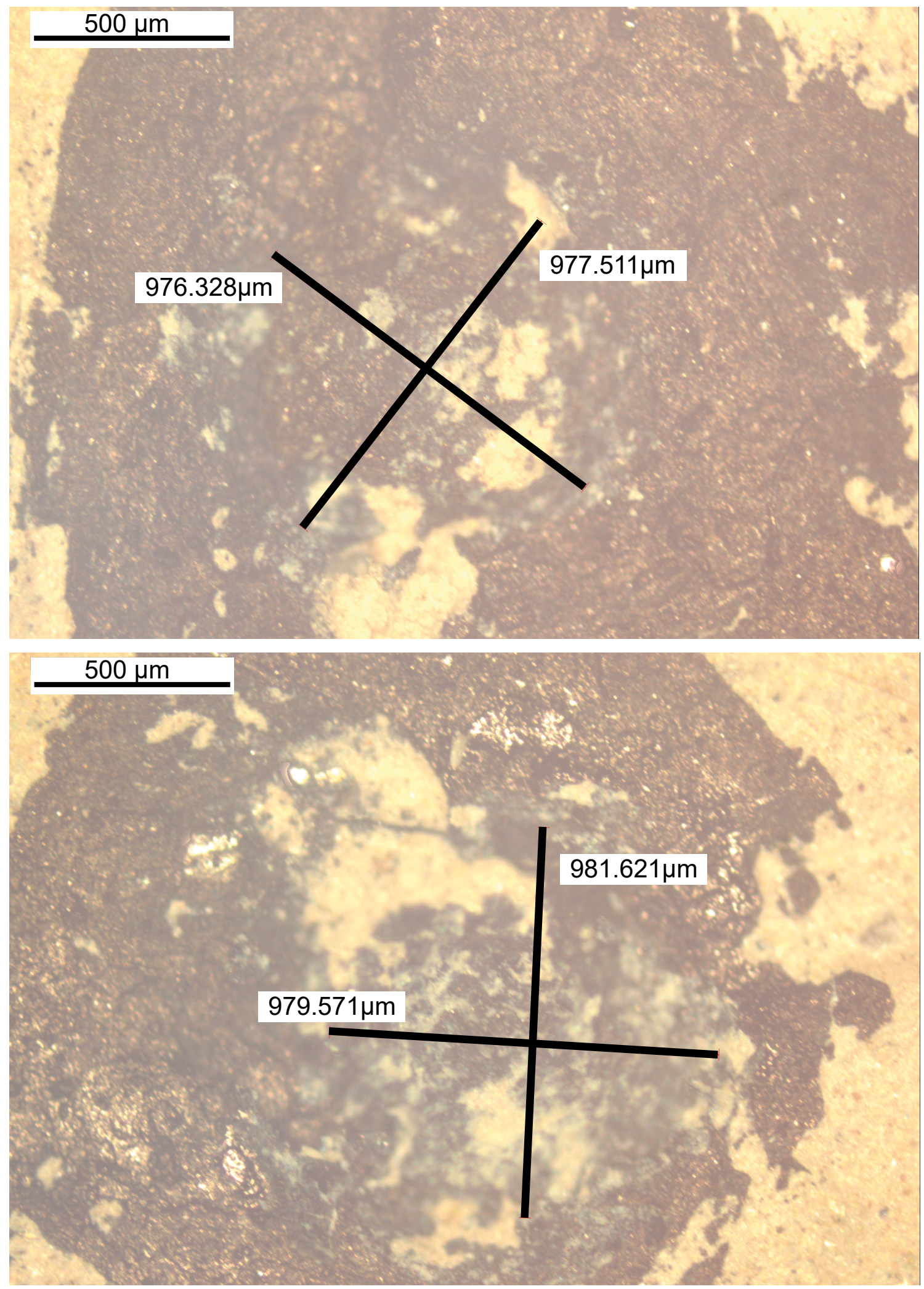

Vick 1.2 (Top) and Vick 2.2 (Bottom). Pictures of the Vickers test. 

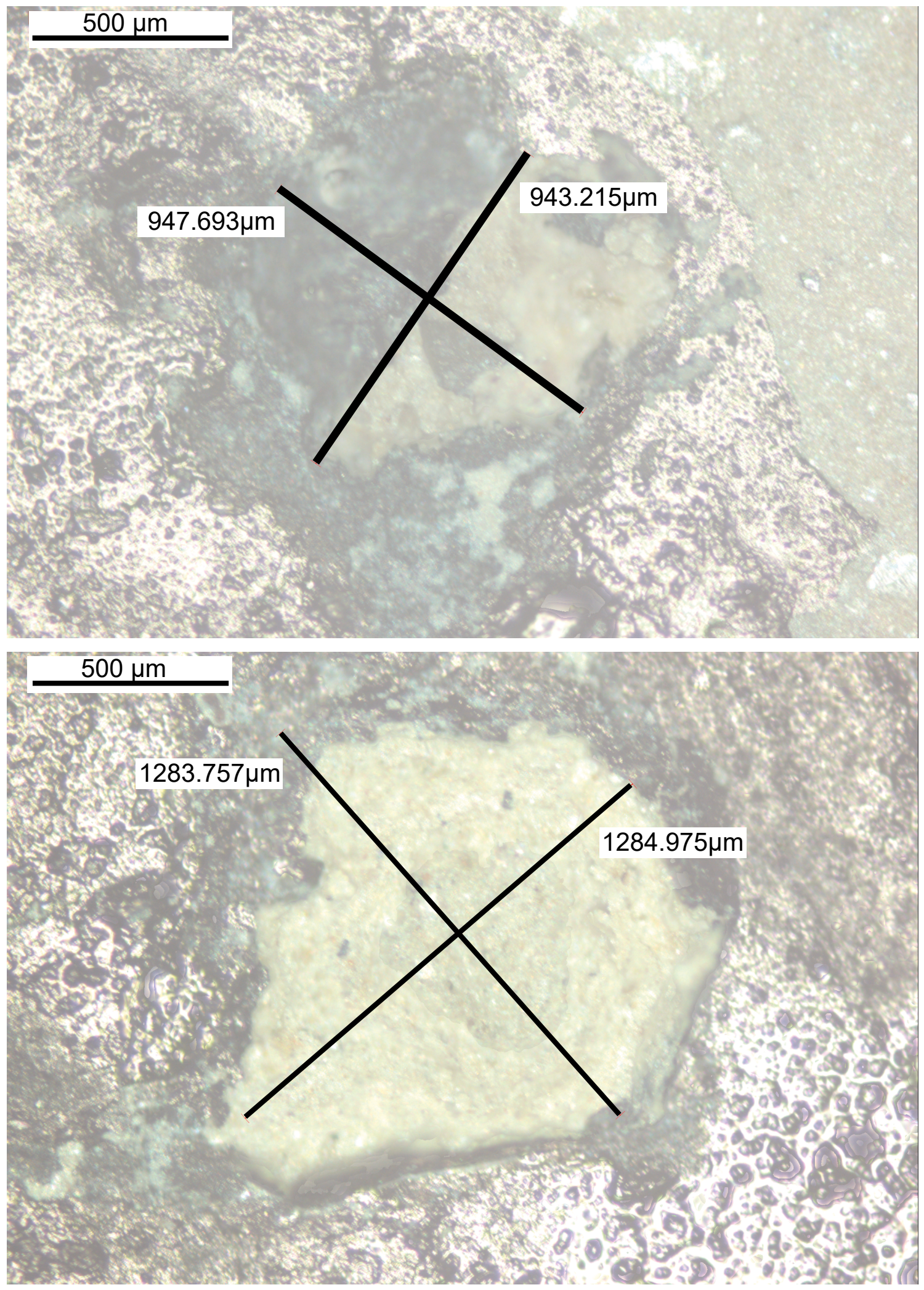

Vick 3.2 (Top) and Vick 4.2 (Bottom). Pictures of the Vickers test. 


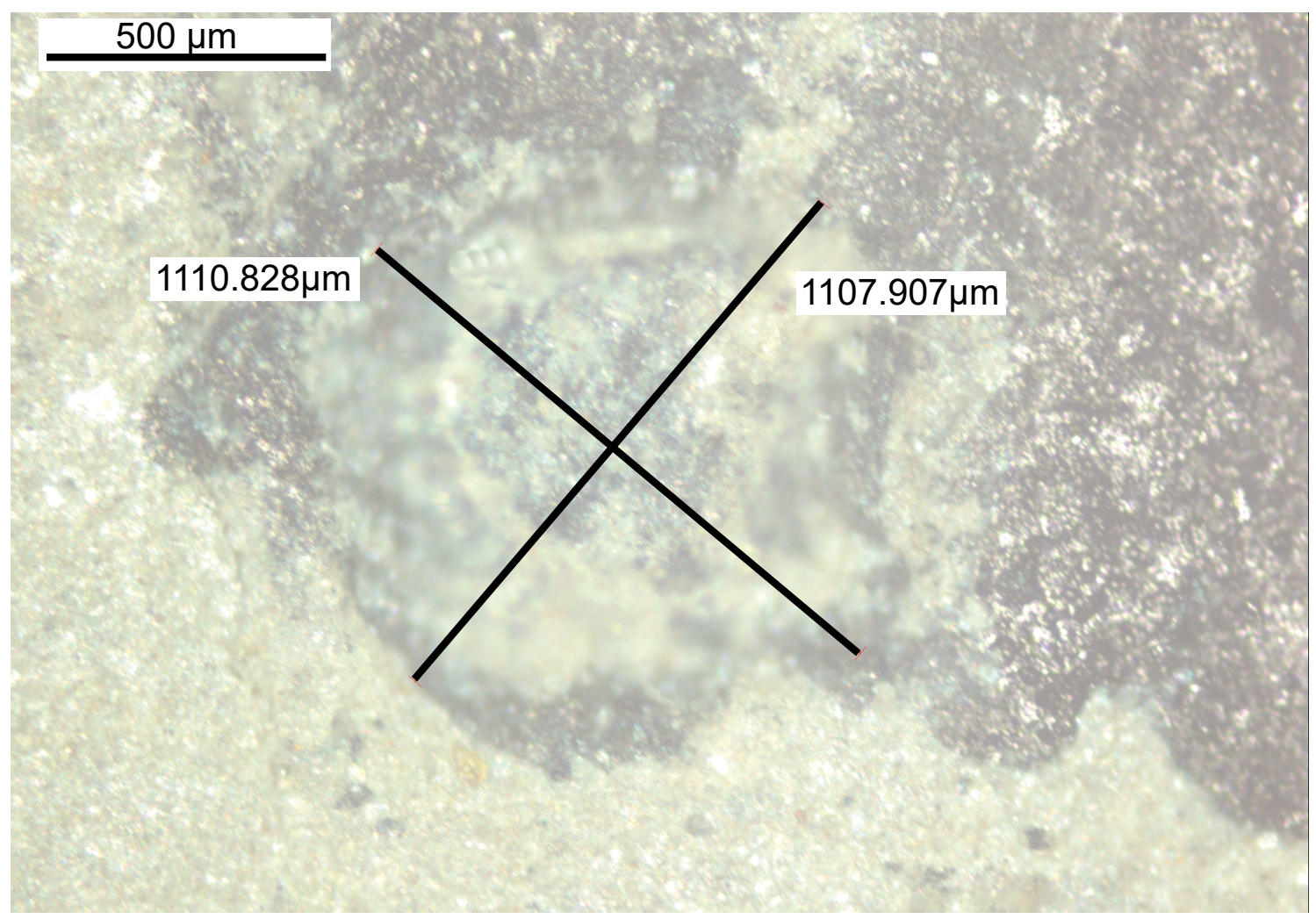

Vick 5.2 Picture of the Vickers test. 

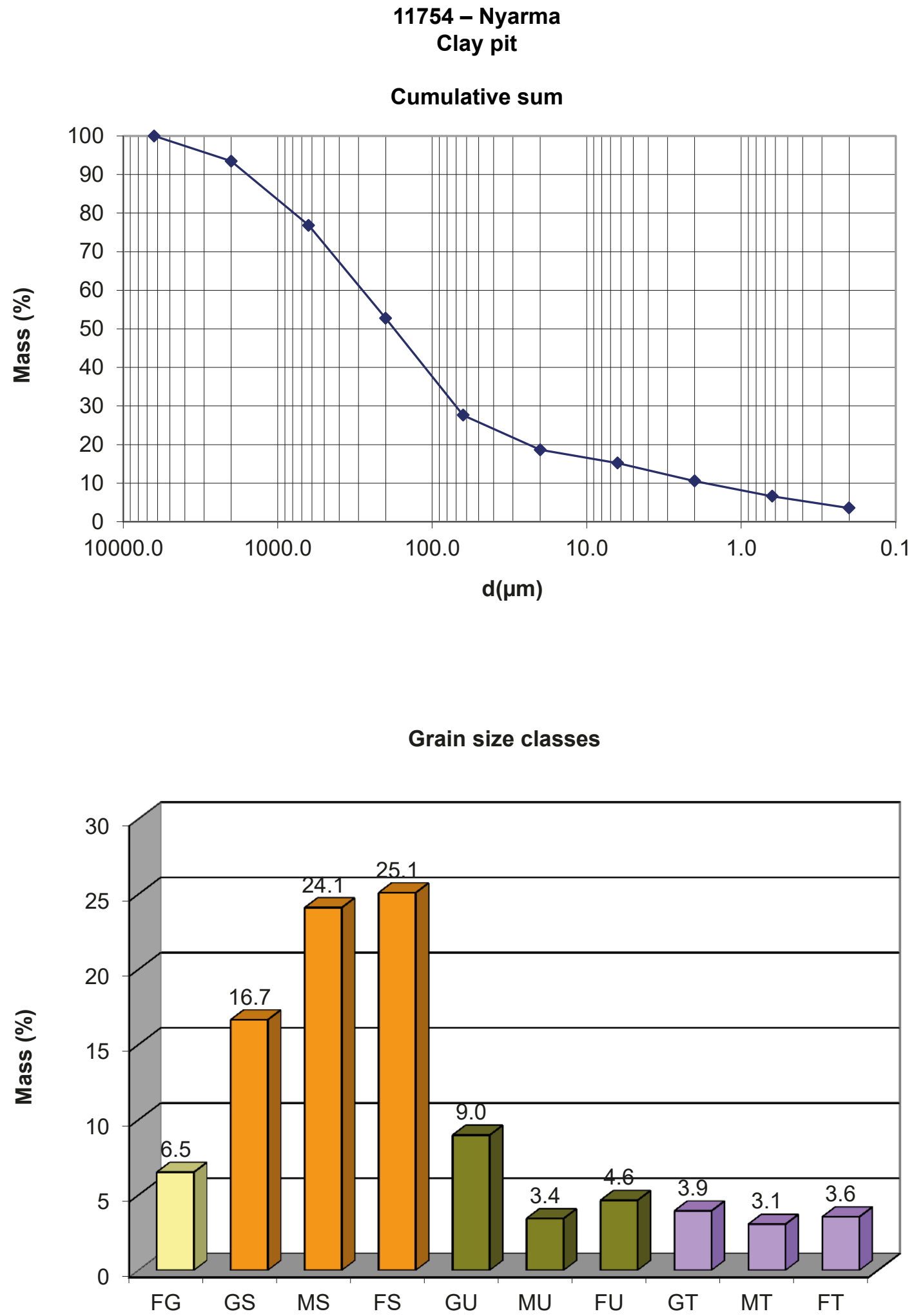

GSD 1.2 Nyarma. Sample 11754. Clay pit.

Top: Cumulative sum. Bottom: Grain size classes. 


\section{6 - Nyarma \\ Clay pit}

\section{Cumulative sum}

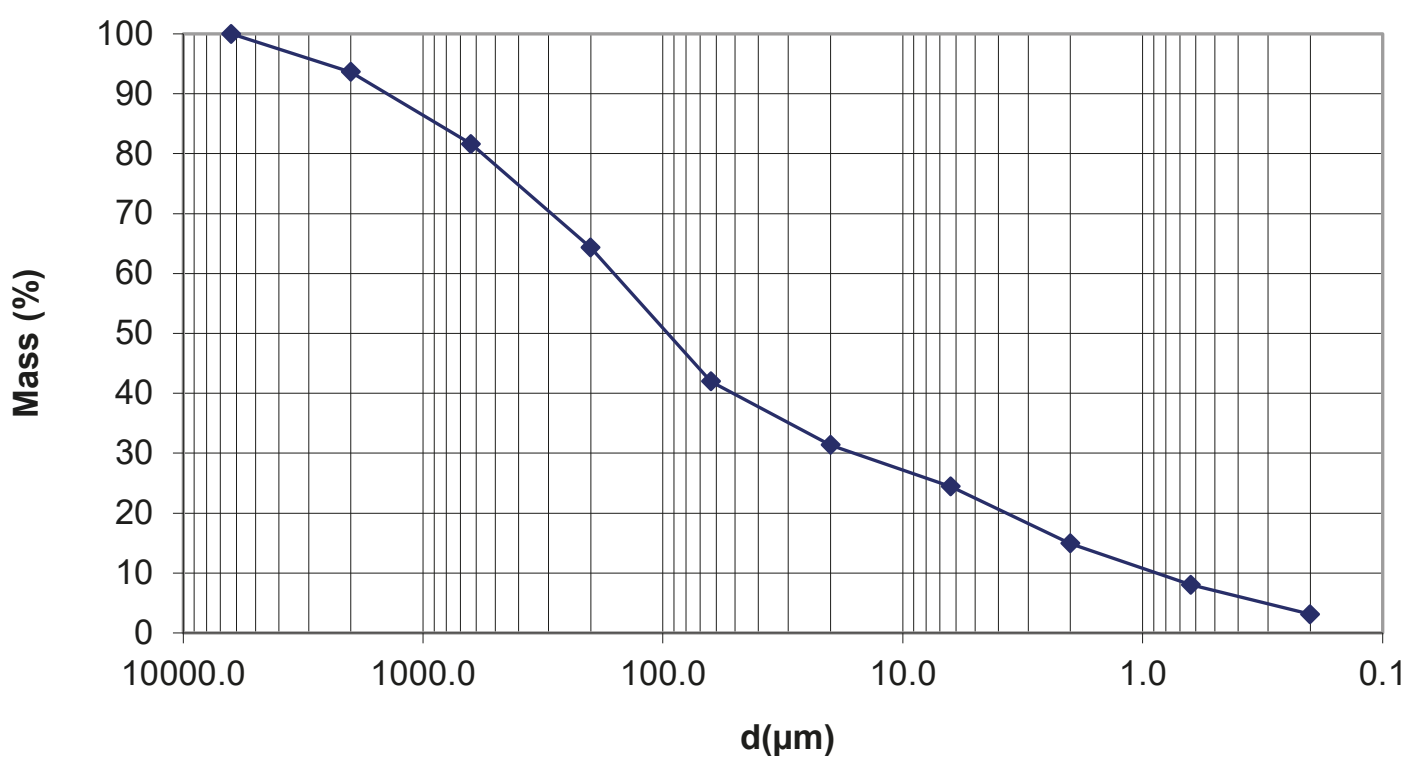

\section{Grain size classes}

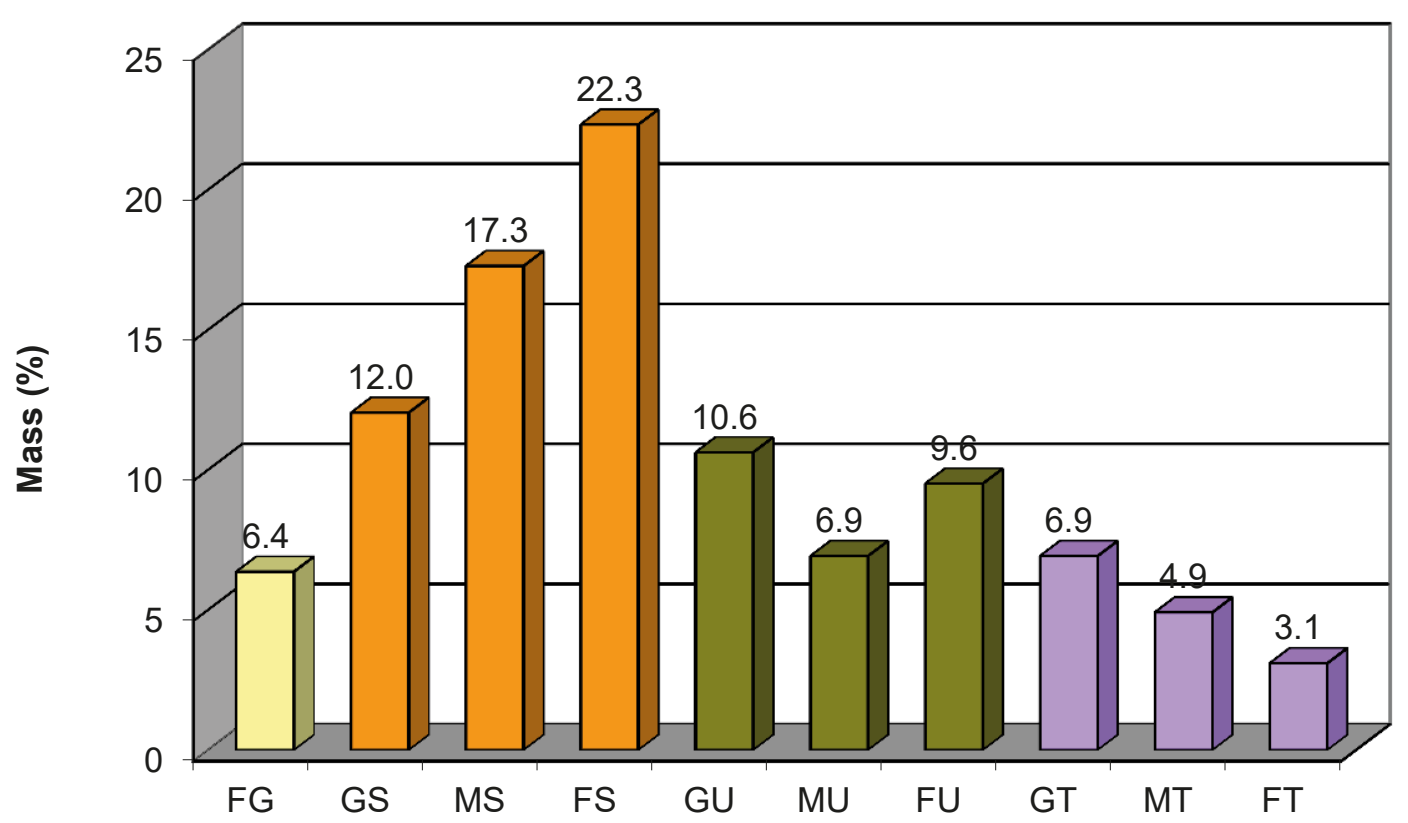

GSD 2.2 Nyarma. Sample 11956. Clay pit.

Top: Cumulative sum. Bottom: Grain size classes. 


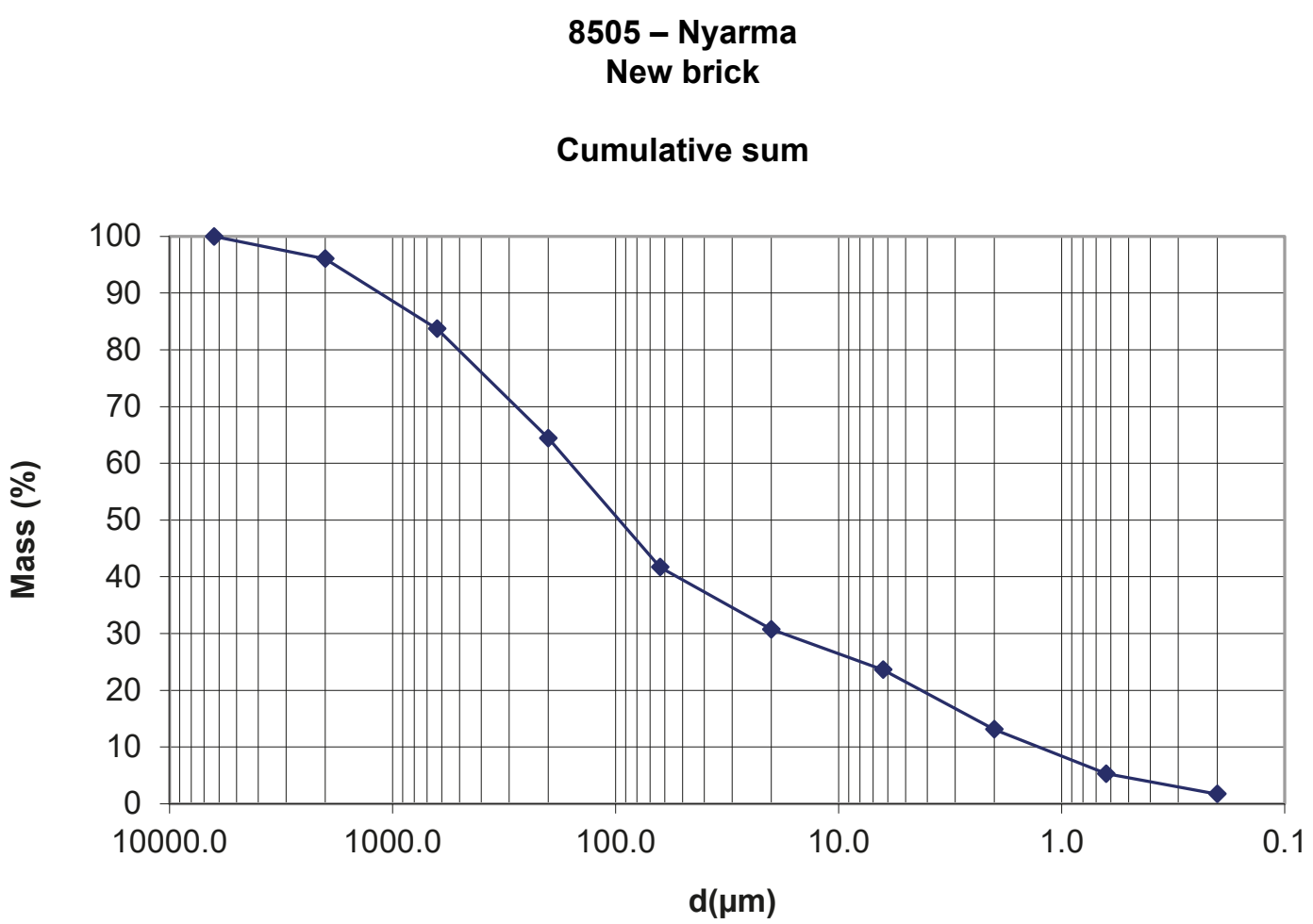

Grain size classes

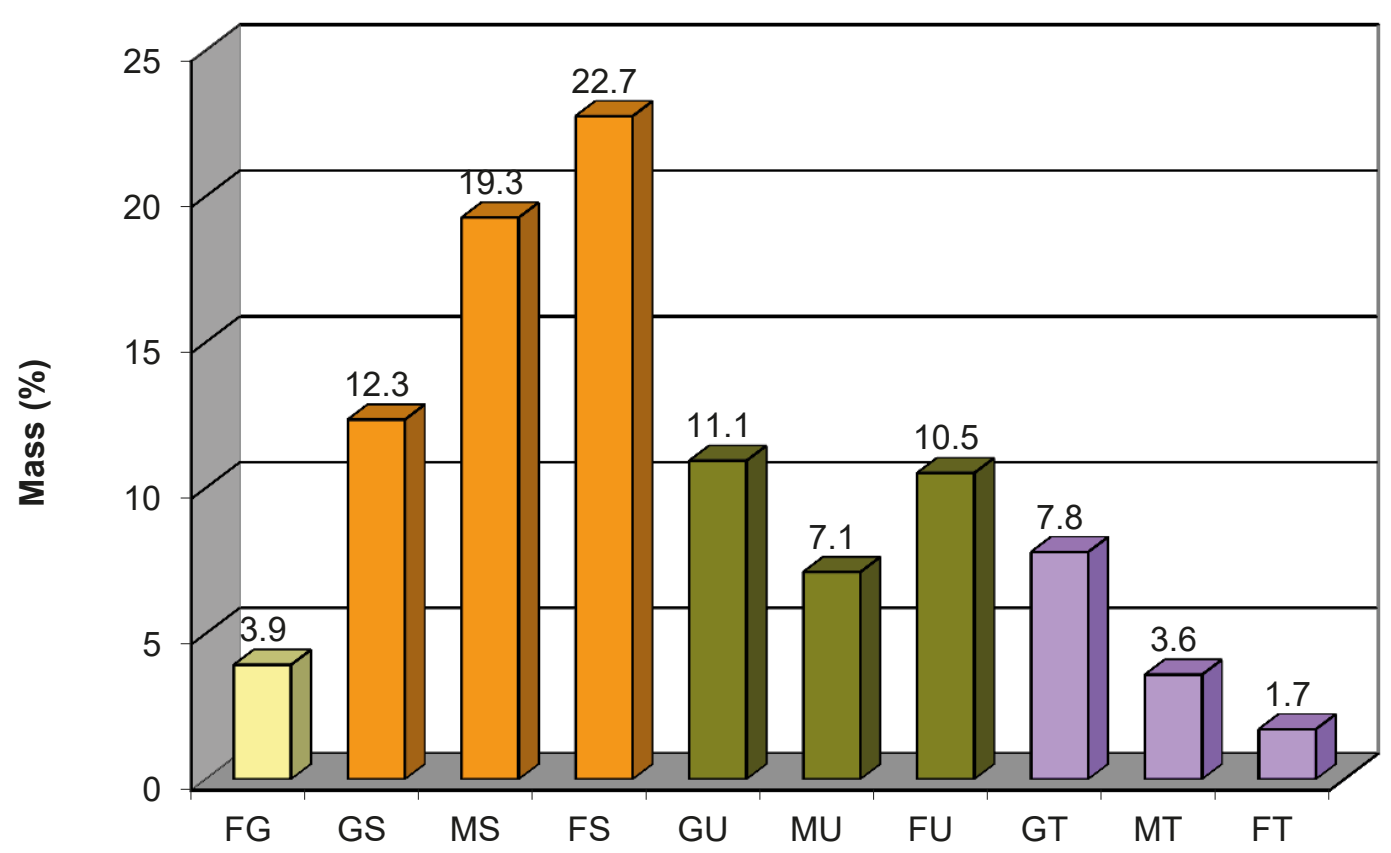

GSD 3.2 Nyarma. Sample 8505. Clay pit.

Top: Cumulative sum. Bottom: Grain size classes. 
11947 - Thikse

New brick

Cumulative sum

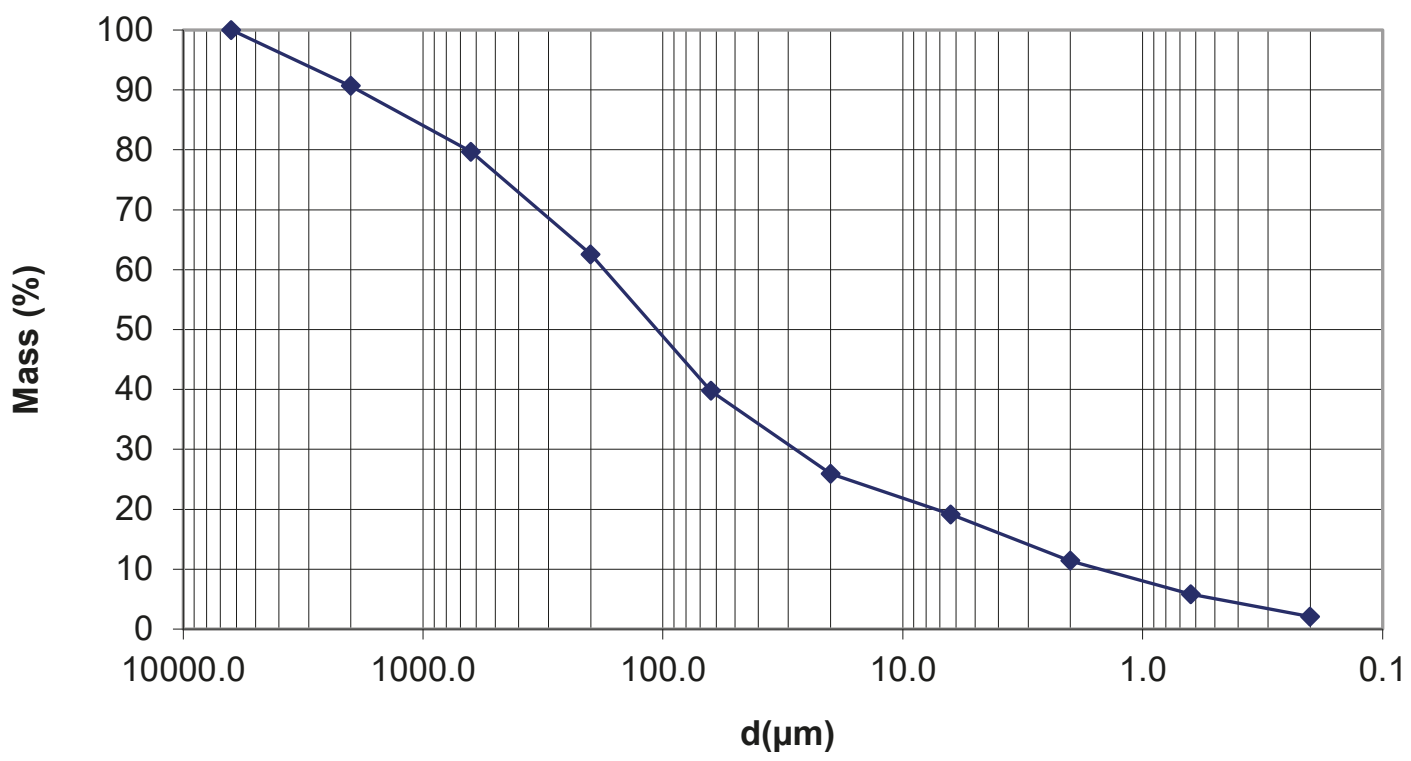

Grain size classes

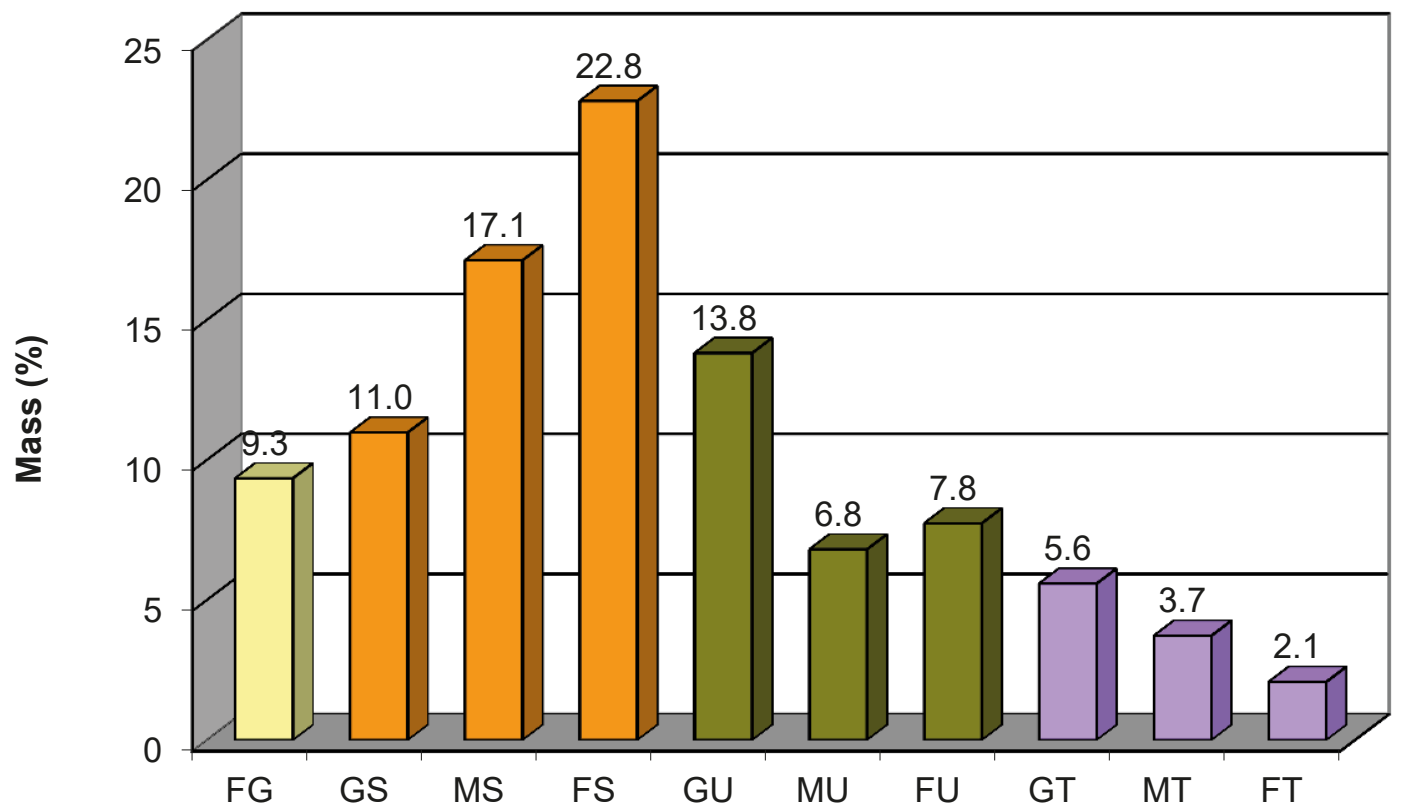

GSD 4.2 Nyarma. Sample 11947. Clay pit.

Top: Cumulative sum. Bottom: Grain size classes. 


\section{3 - Nyarma. Temple I. Cella Interior plaster}

\section{Cumulative sum}

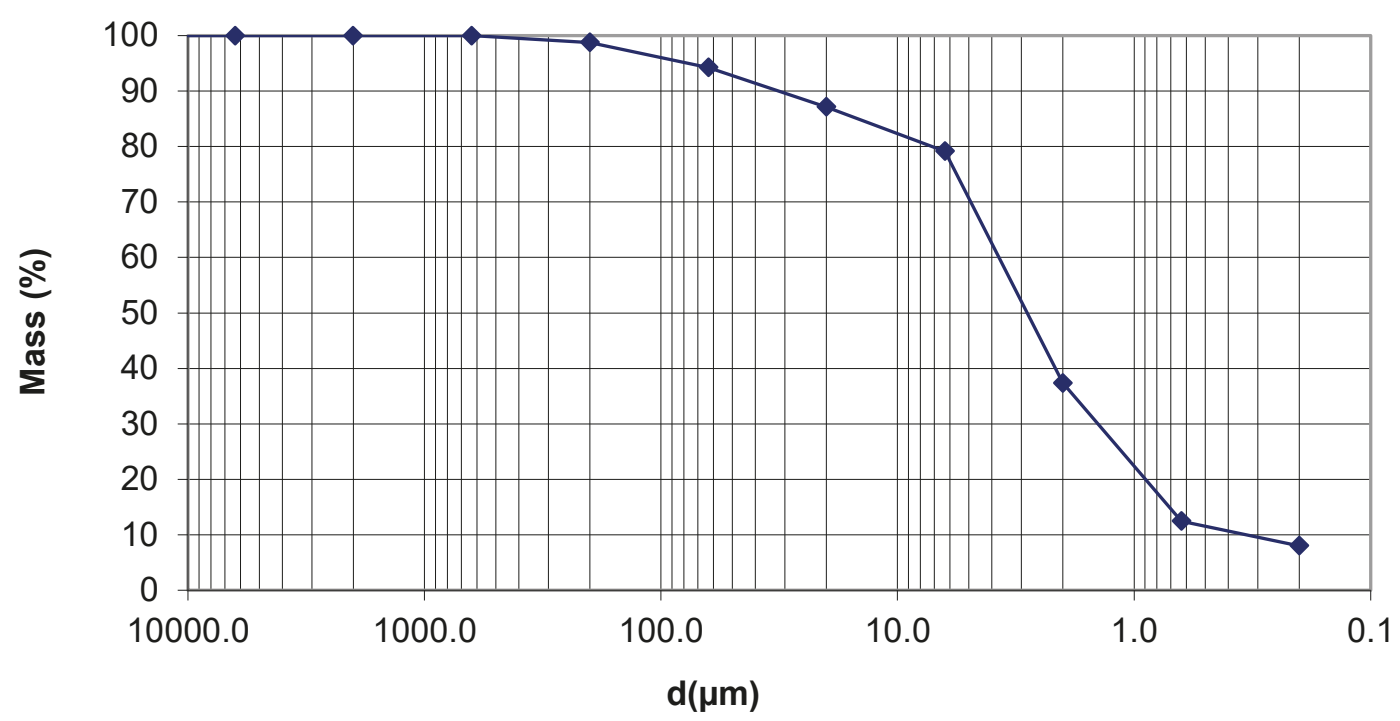

\section{Grain size classes}

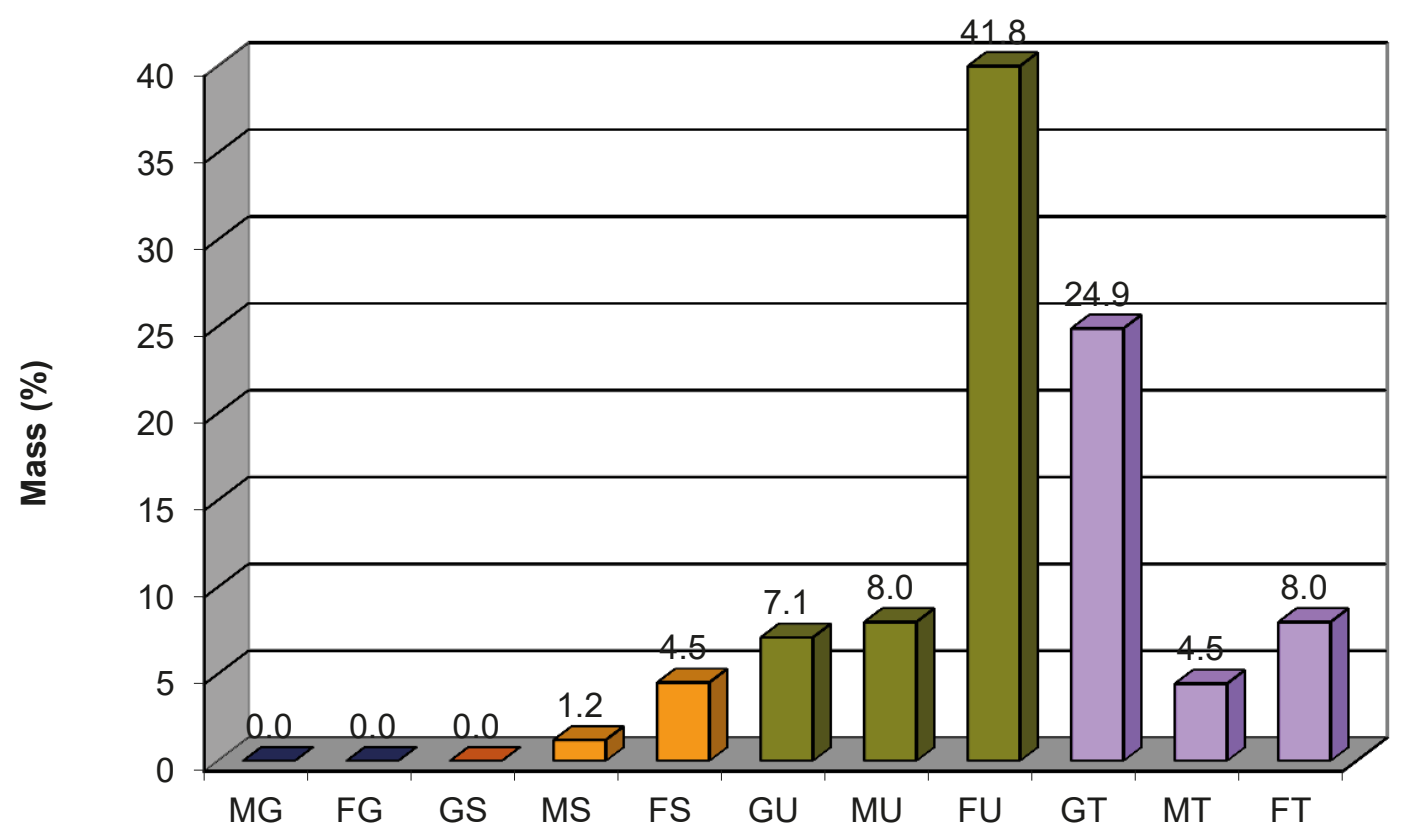

GSD 5.2 Nyarma. Sample 6073. Temple I. Main temple. Cella. Interior wall plaster. Top: Cumulative sum. Bottom: Grain size classes. 


\section{5 - Nyarma. Temple I. Cella Interior wall plaster}

\section{Cumulative sum}
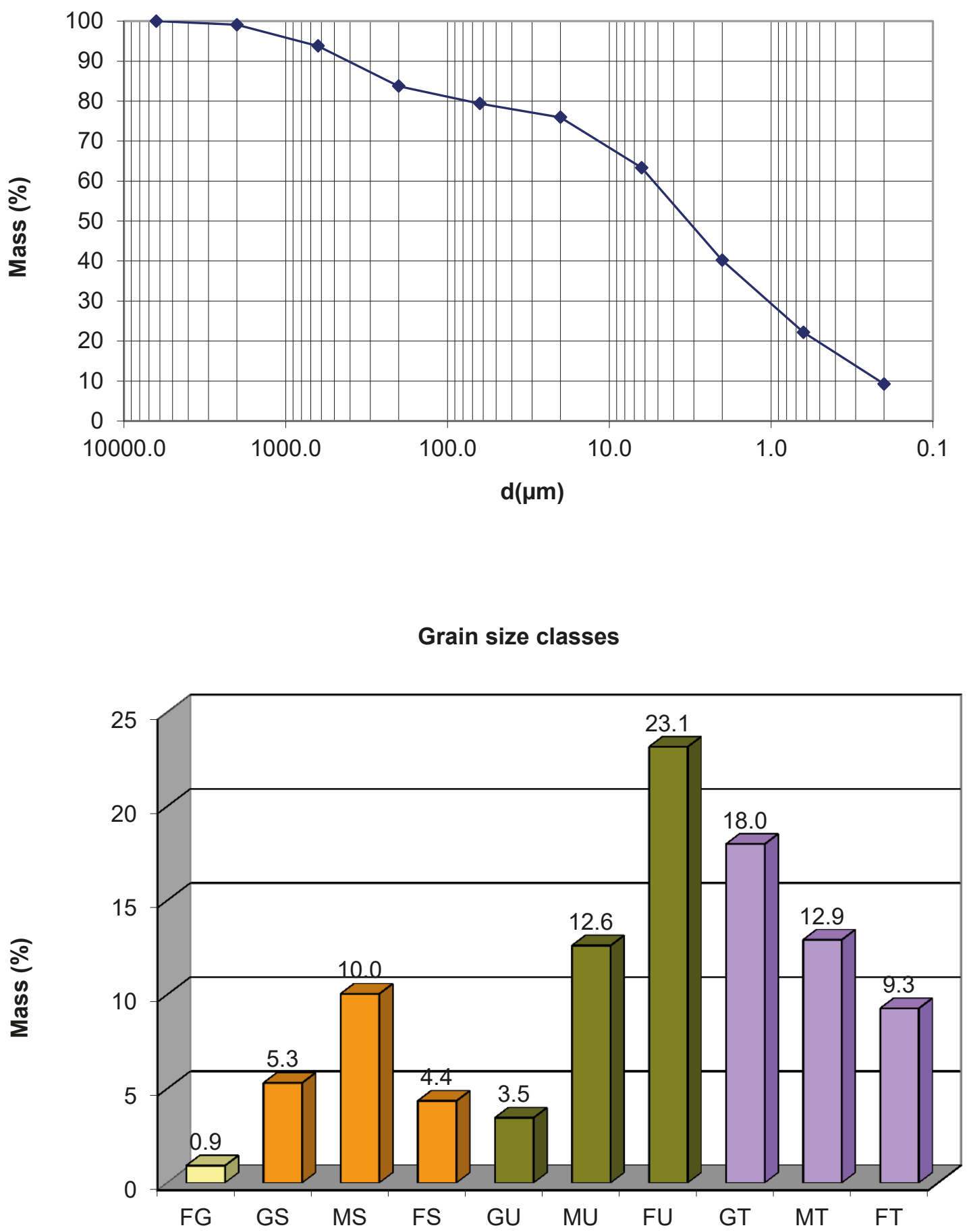

GSD 6.2 Nyarma. Sample 11745. Temple I. Cella. Interior wall plaster.

Top: Cumulative sum. Bottom: Grain size classes. 


\section{3 - Nyarma. Temple I. Cella Interior wall plaster}

\section{Cumulative sum}

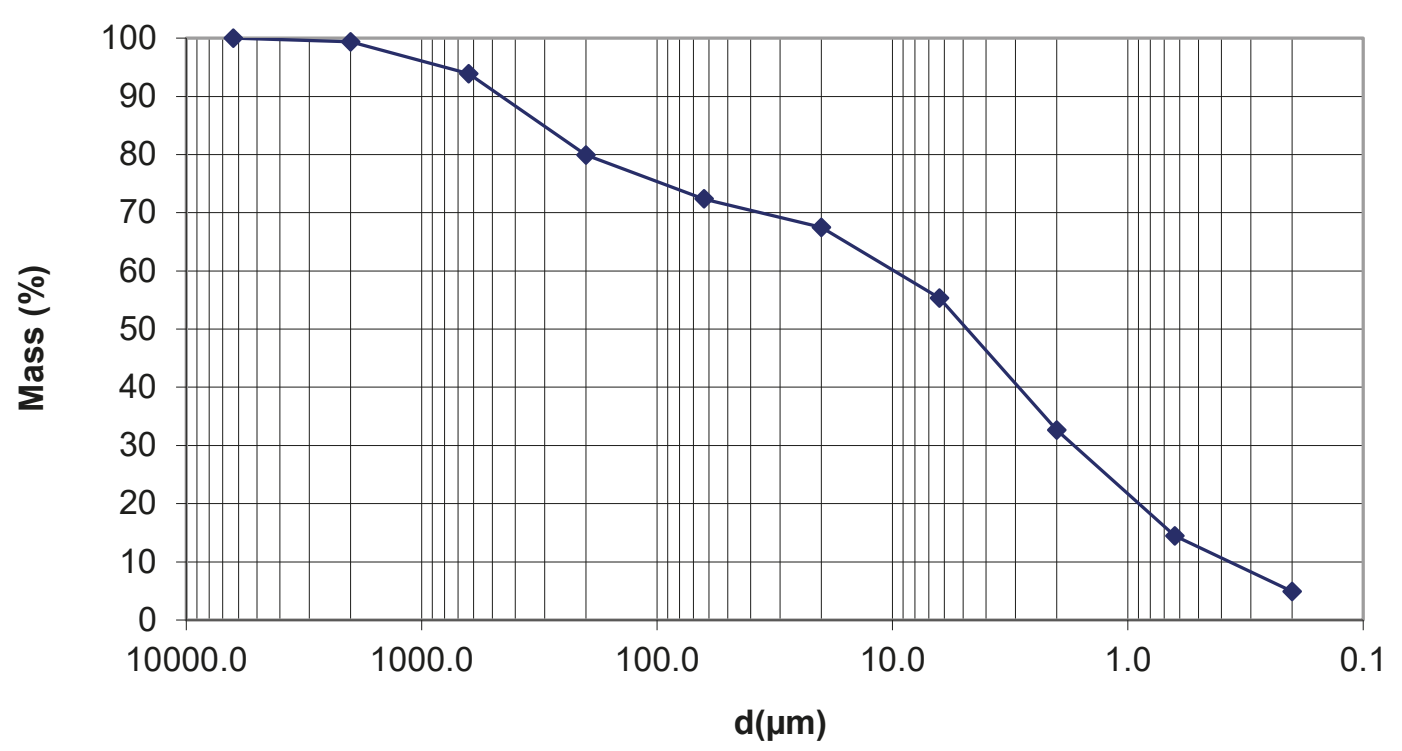

Grain size classes

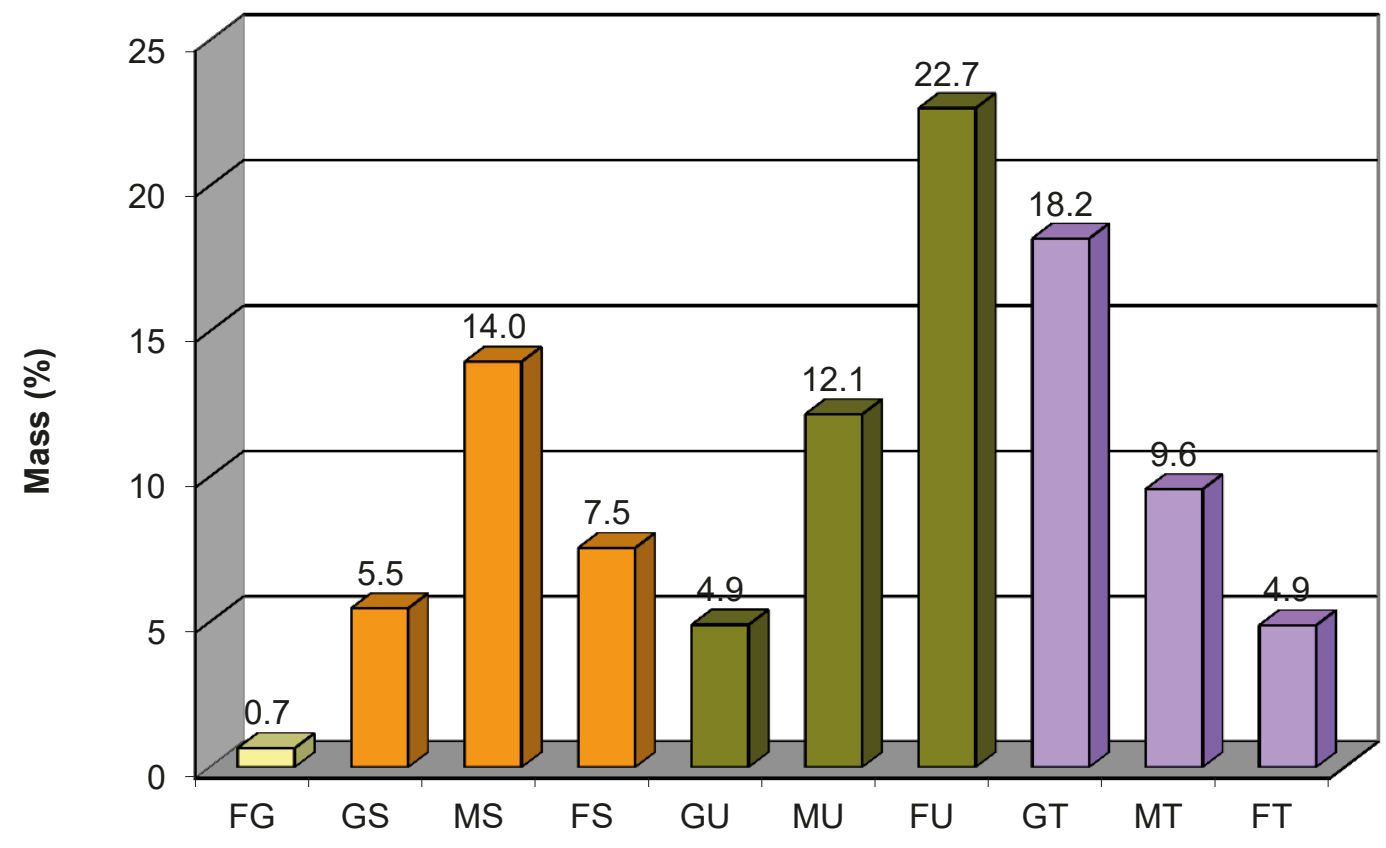

GSD 7.2 Nyarma. Sample 11753. Temple I. Cella. Interior wall plaster.

Top: Cumulative sum. Bottom: Grain size classes. 
11925 - Nyarma. Temple I. Cella Interior wall plaster

Cumulative sum

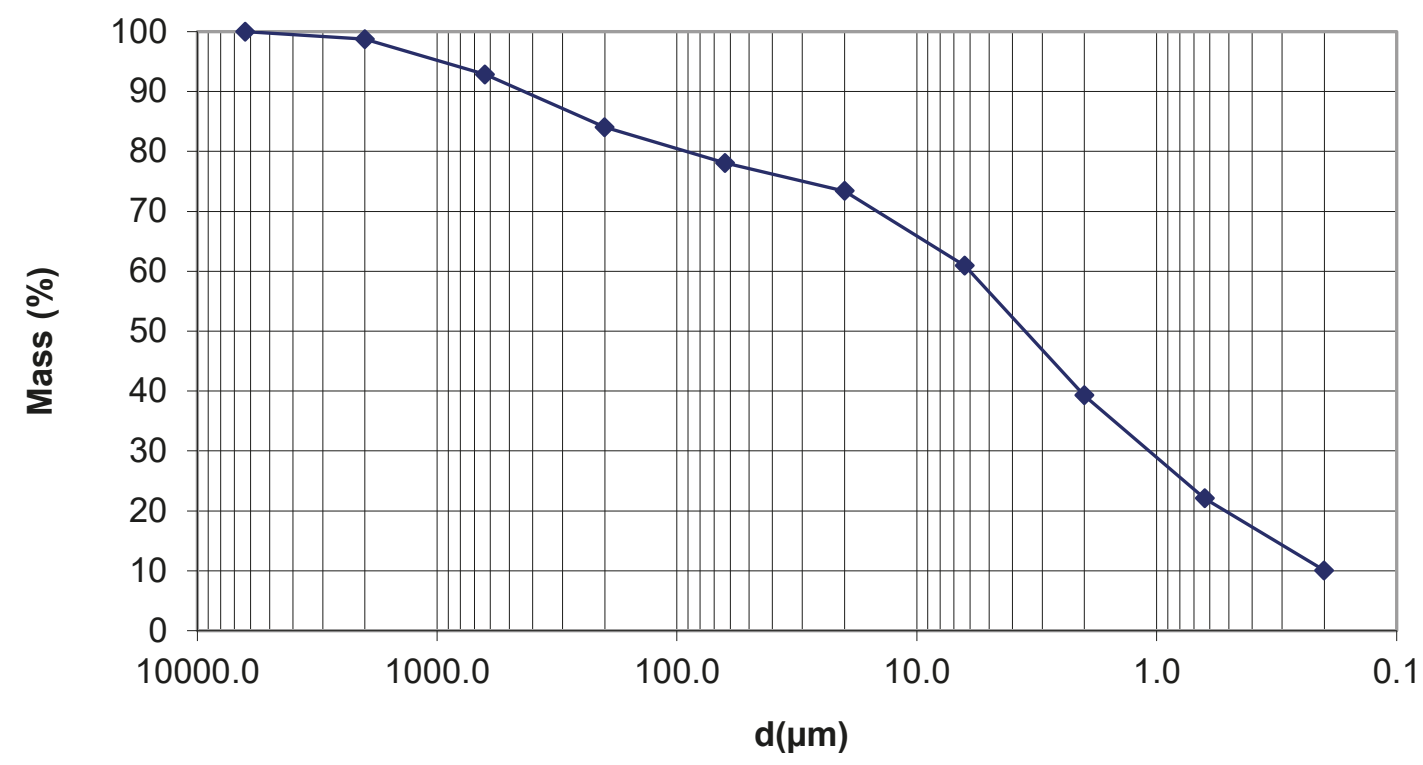

Grain size classes

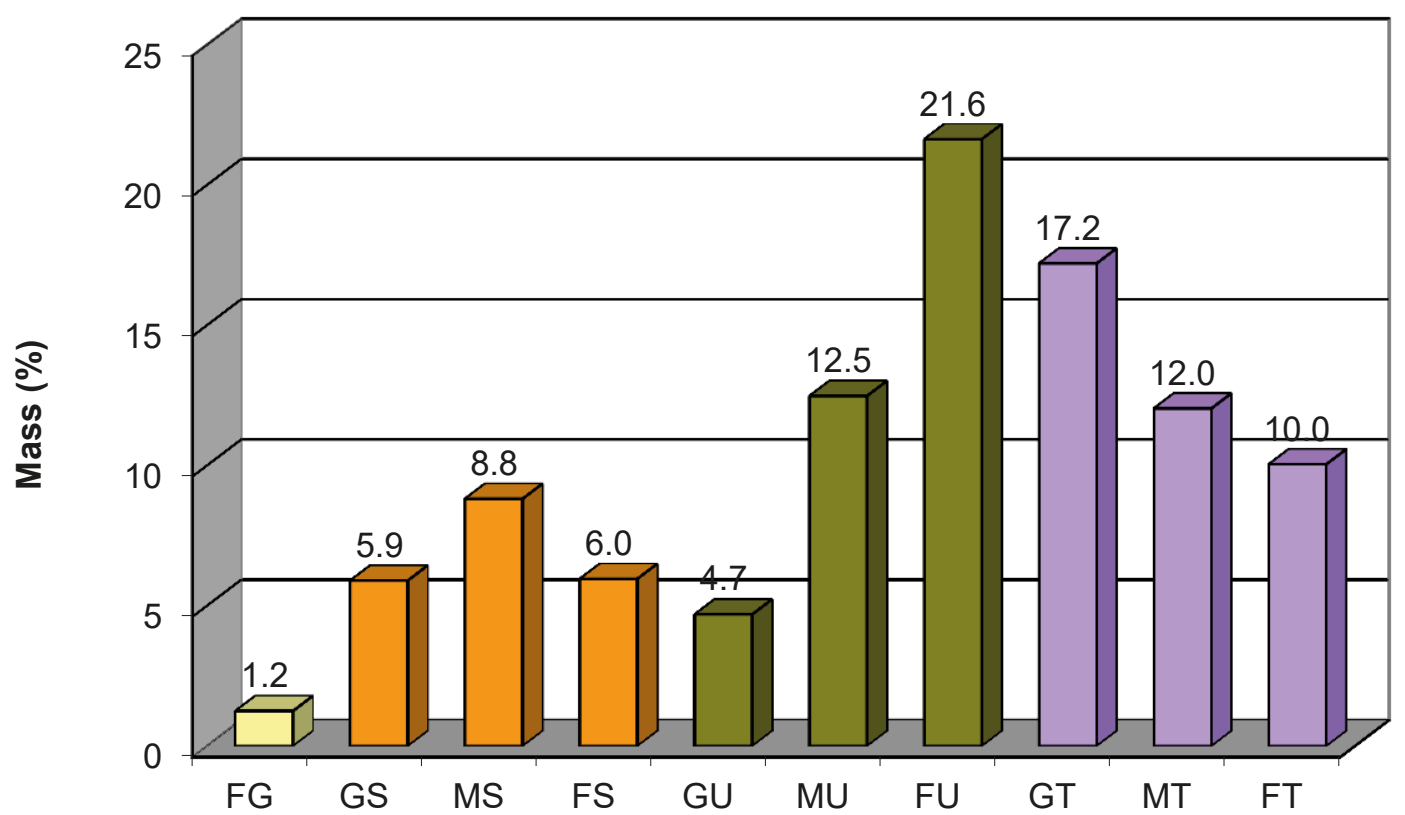

GSD 8.2 Nyarma. Sample 11925. Temple I. Cella. Interior wall plaster.

Top: Cumulative sum. Bottom: Grain size classes. 
11918 - Nyarma. Temple I. Assembly hall Interior wall plaster

Cumulative sum

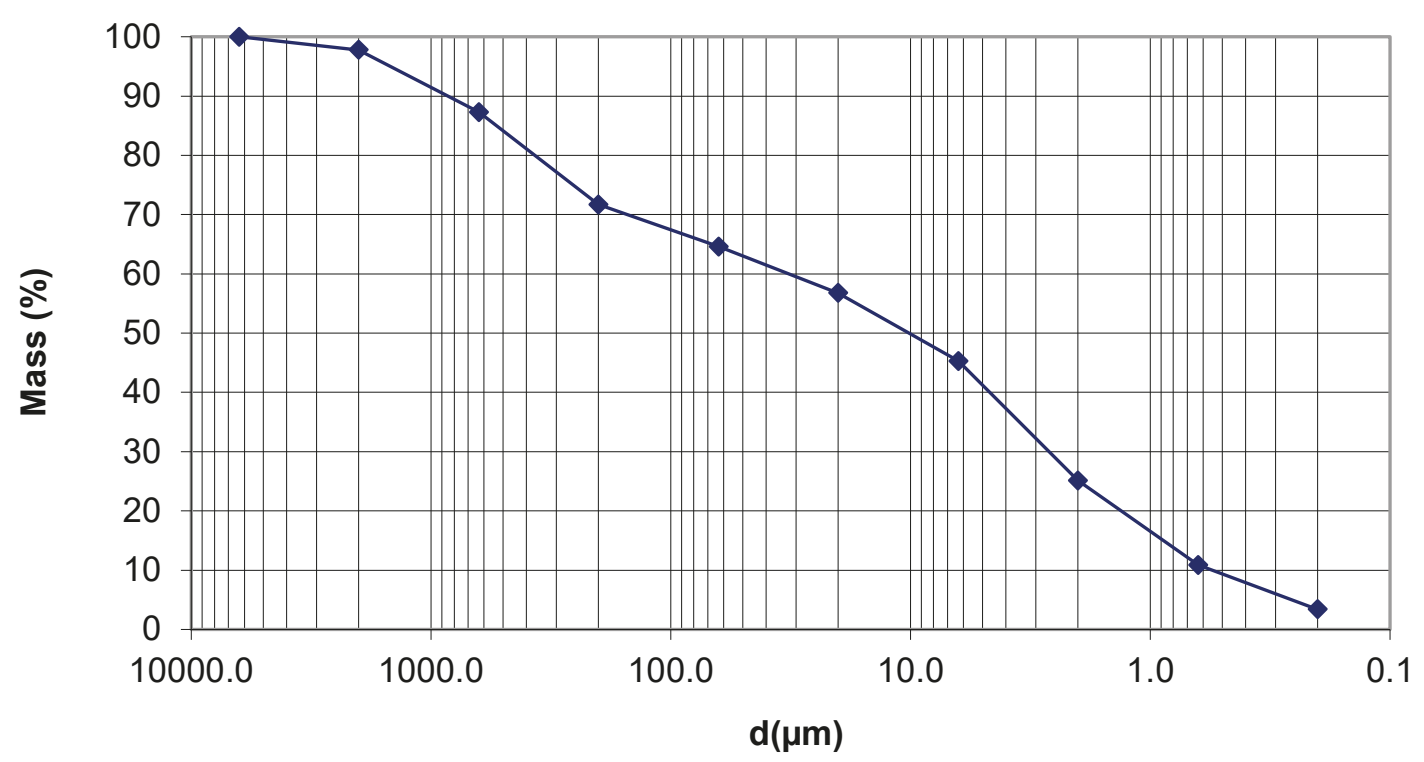

Grain size classes

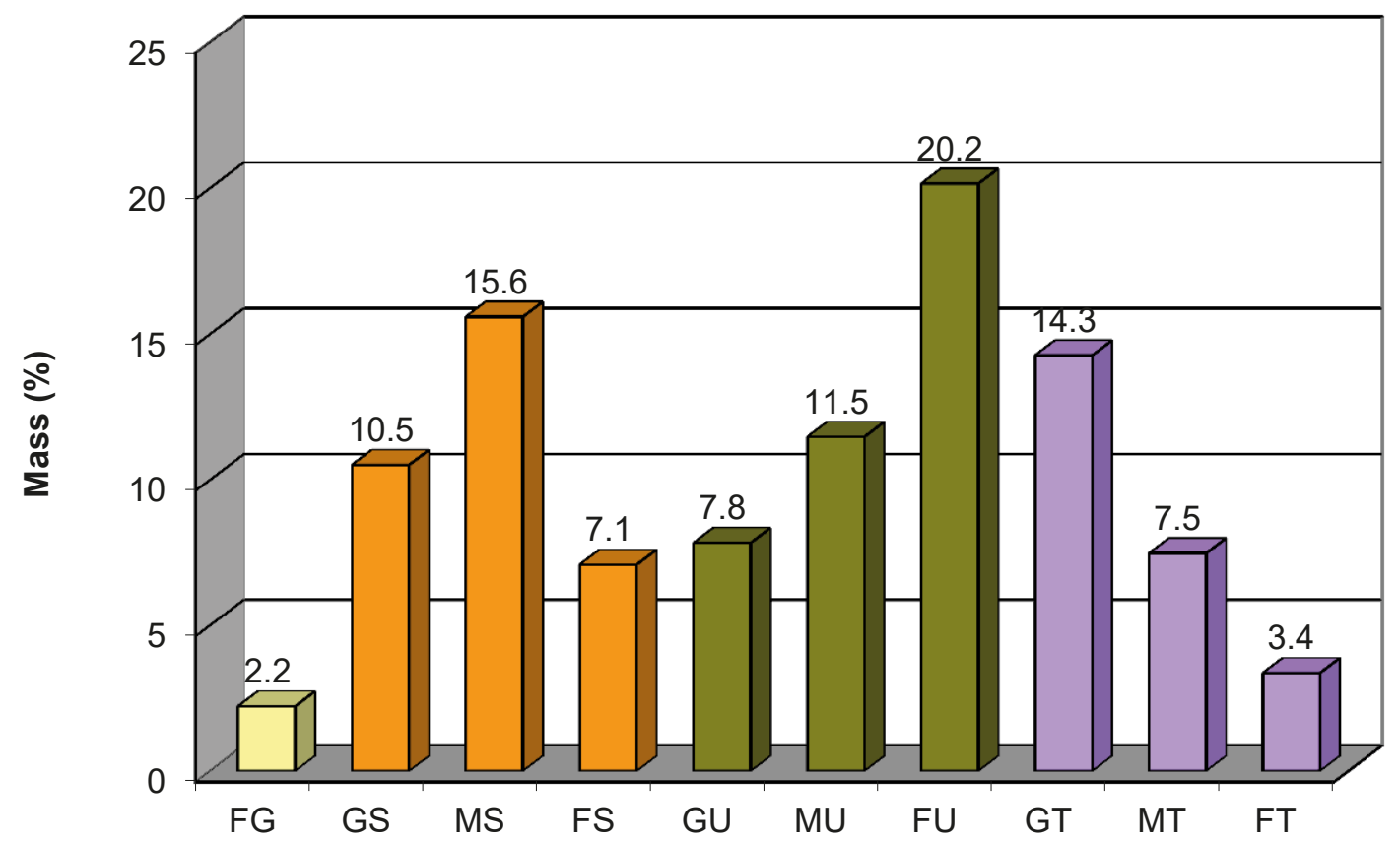

GSD 9.2 Nyarma. Sample 11918. Temple I. Assembly hall. Interior wall plaster.

Top: Cumulative sum. Bottom: Grain size classes. 


\section{1 - Nyarma. Temple I. Assembly hall Interior wall plaster}

Cumulative sum

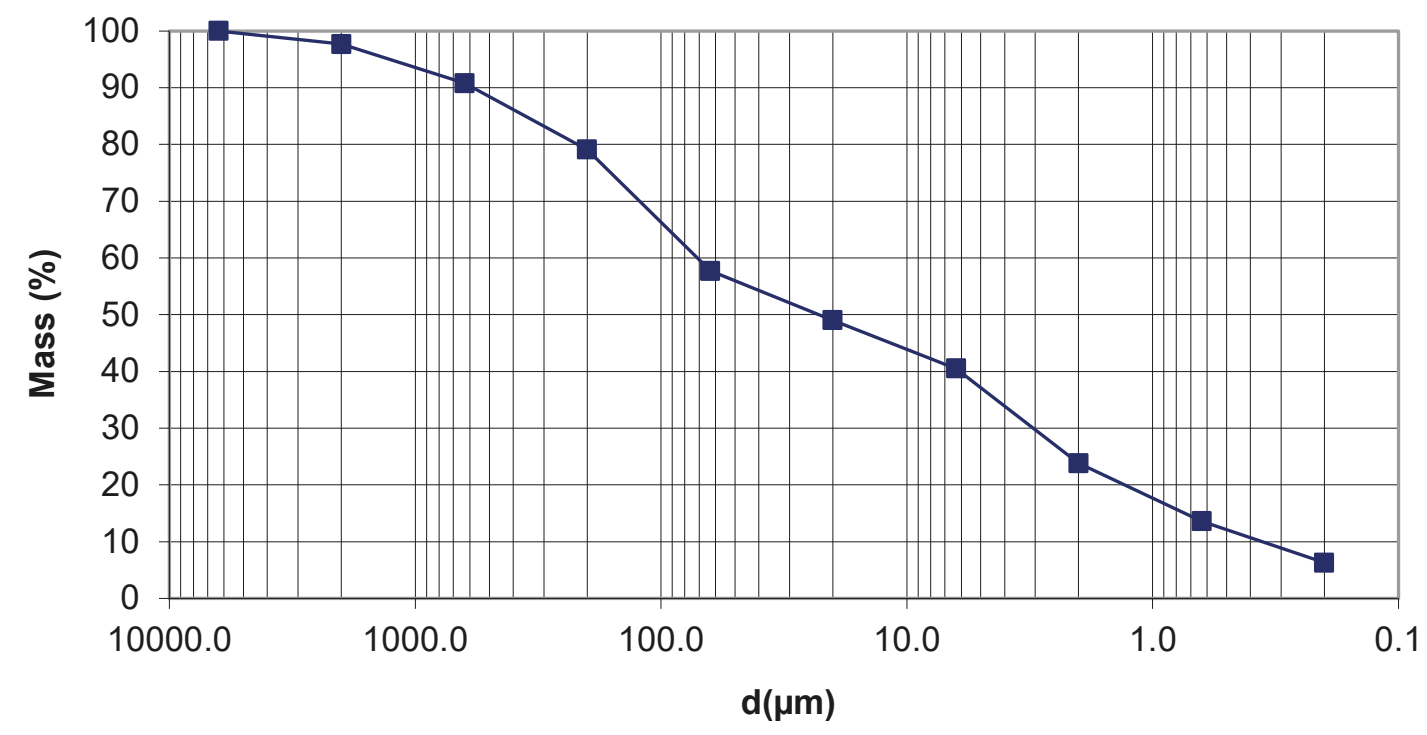

Grain size classes

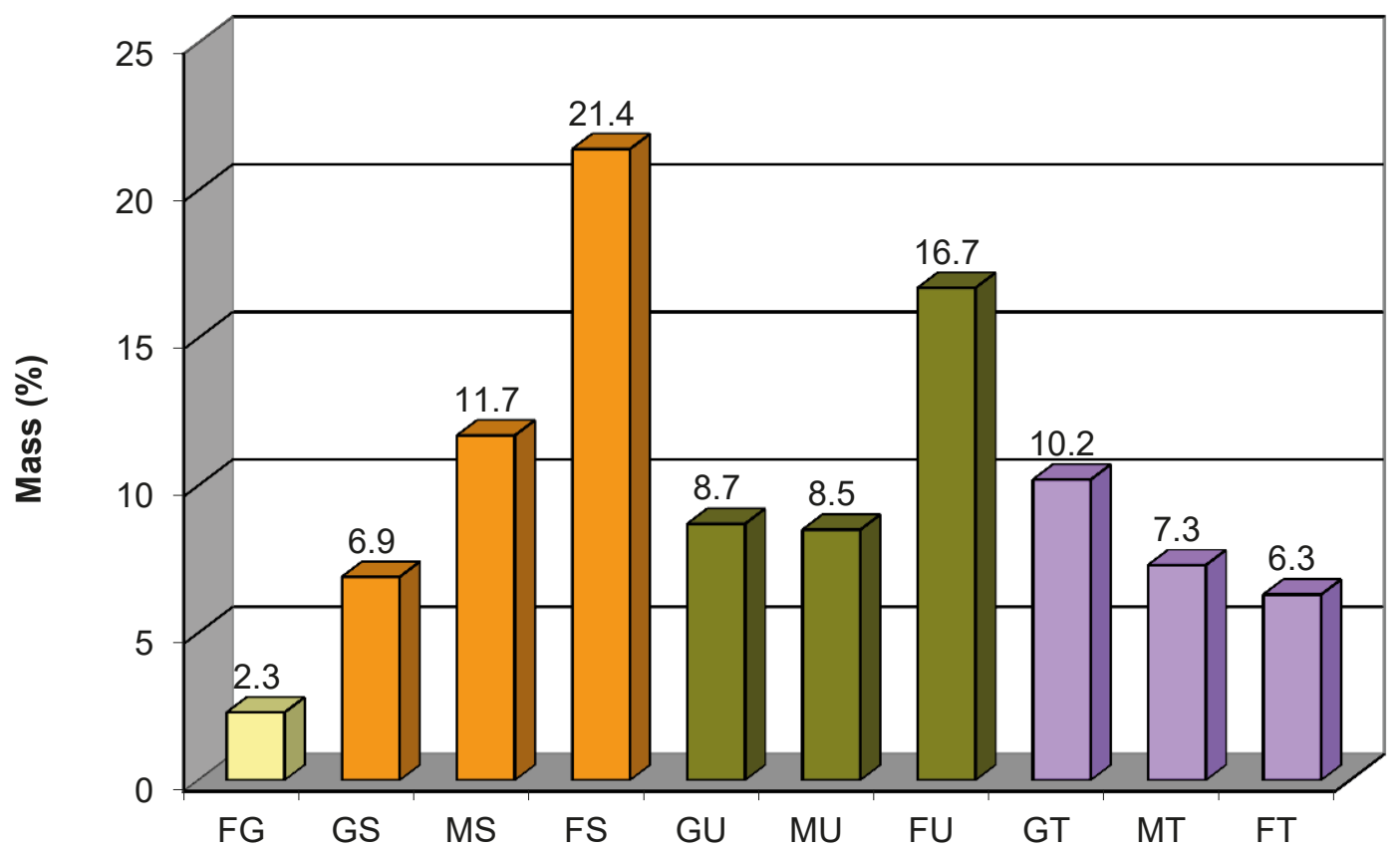

GSD 10.2 Nyarma. Sample 8531. Temple I. Assembly hall. Interior wall plaster. Top: Cumulative sum. Bottom: Grain size classes. 


\section{4 - Nyarma. Temple IIla Interior wall plaster}

Cumulative sum

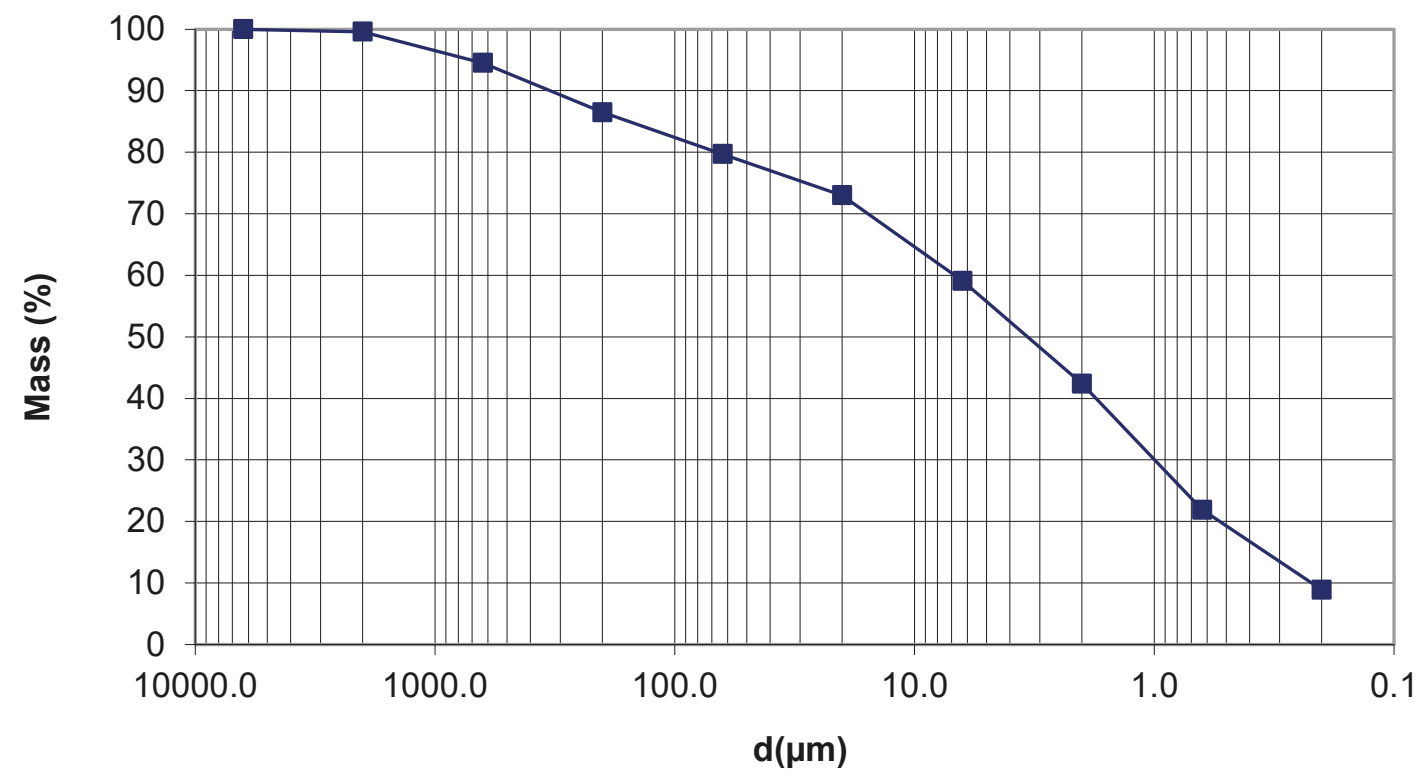

Grain size classes

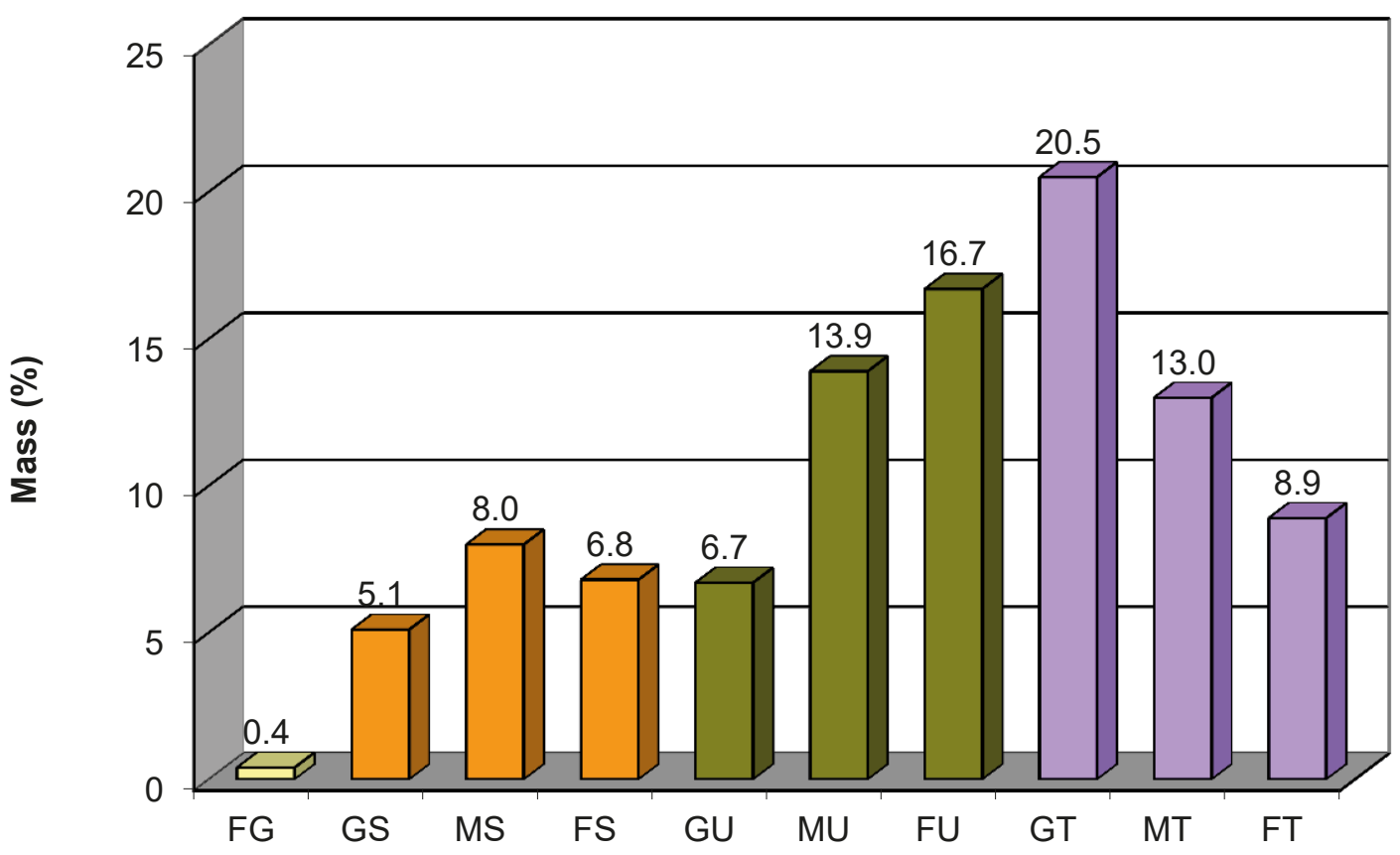

GSD 11.2 Nyarma. Sample 8514. Temple IIIa. Interior wall plaster.

Top: Cumulative sum. Bottom: Grain size classes. 
15406 - Nyarma. Temple IV Interior wall plaster. Upper layer

Cumulative sum

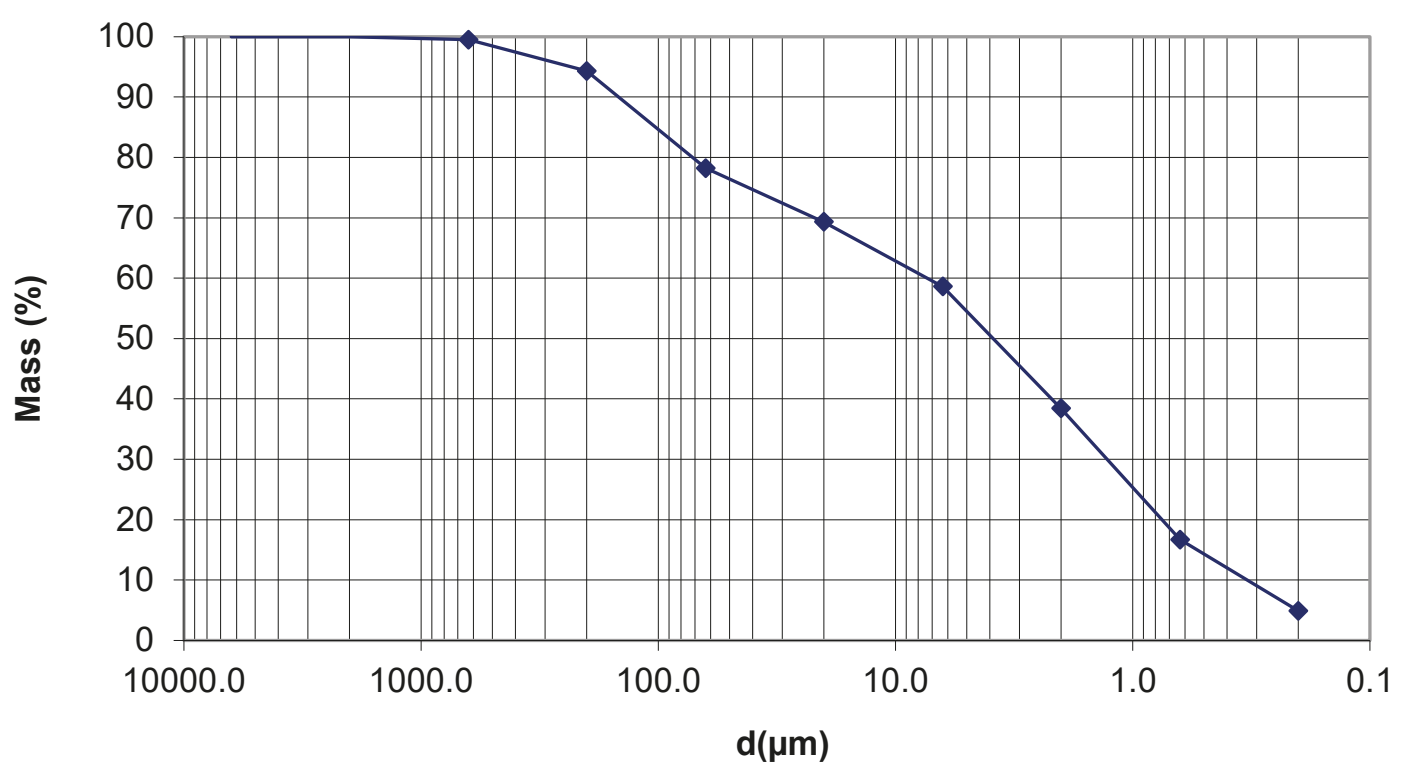

Grain size classes

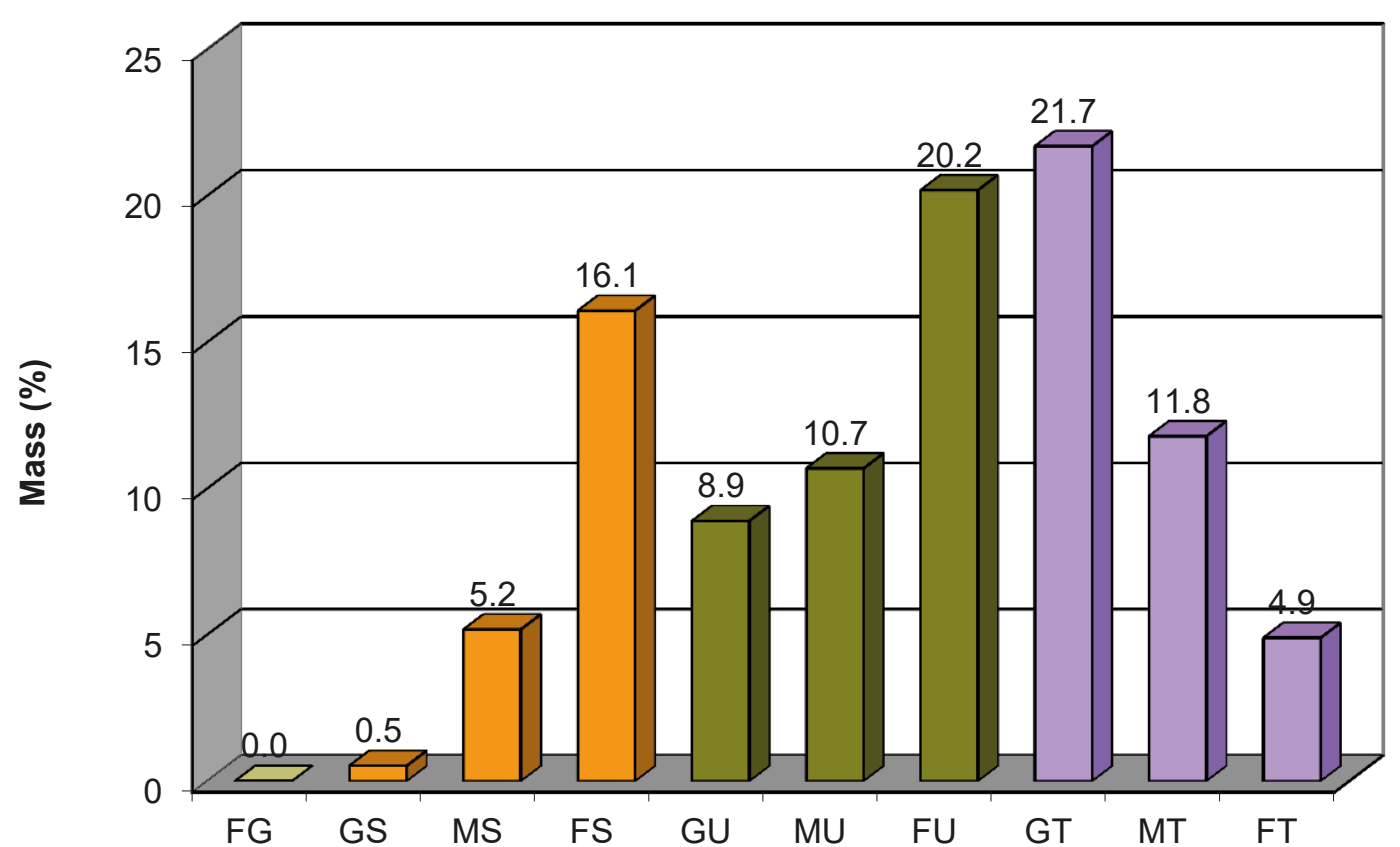

GSD 12.2 Nyarma. Sample 15406. Temple IV. Interior wall plaster. Upper layer.

Top: Cumulative sum. Bottom: Grain size classes. 
8517 - Nyarma. Temple IV

Interior wall plaster. Ground layer

Cumulative sum

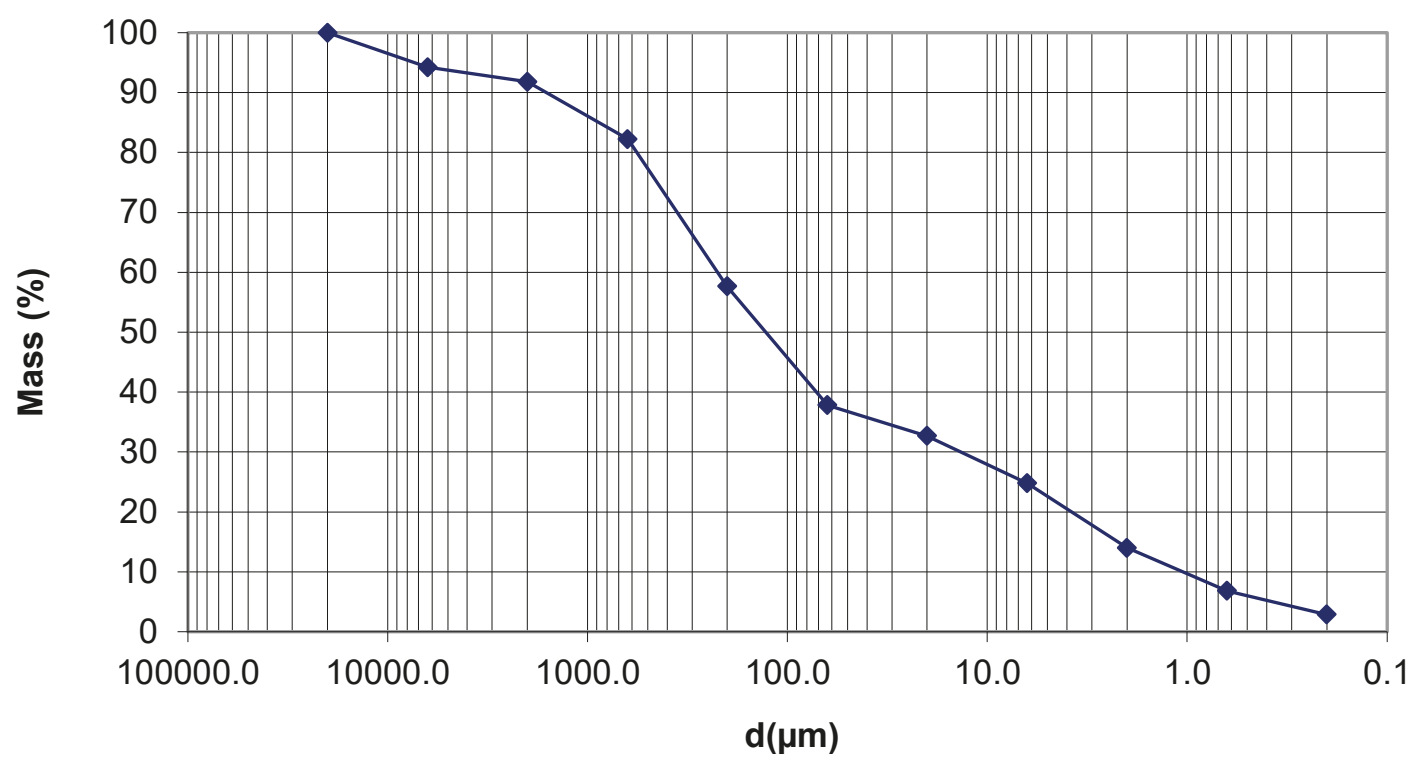

Grain size classes

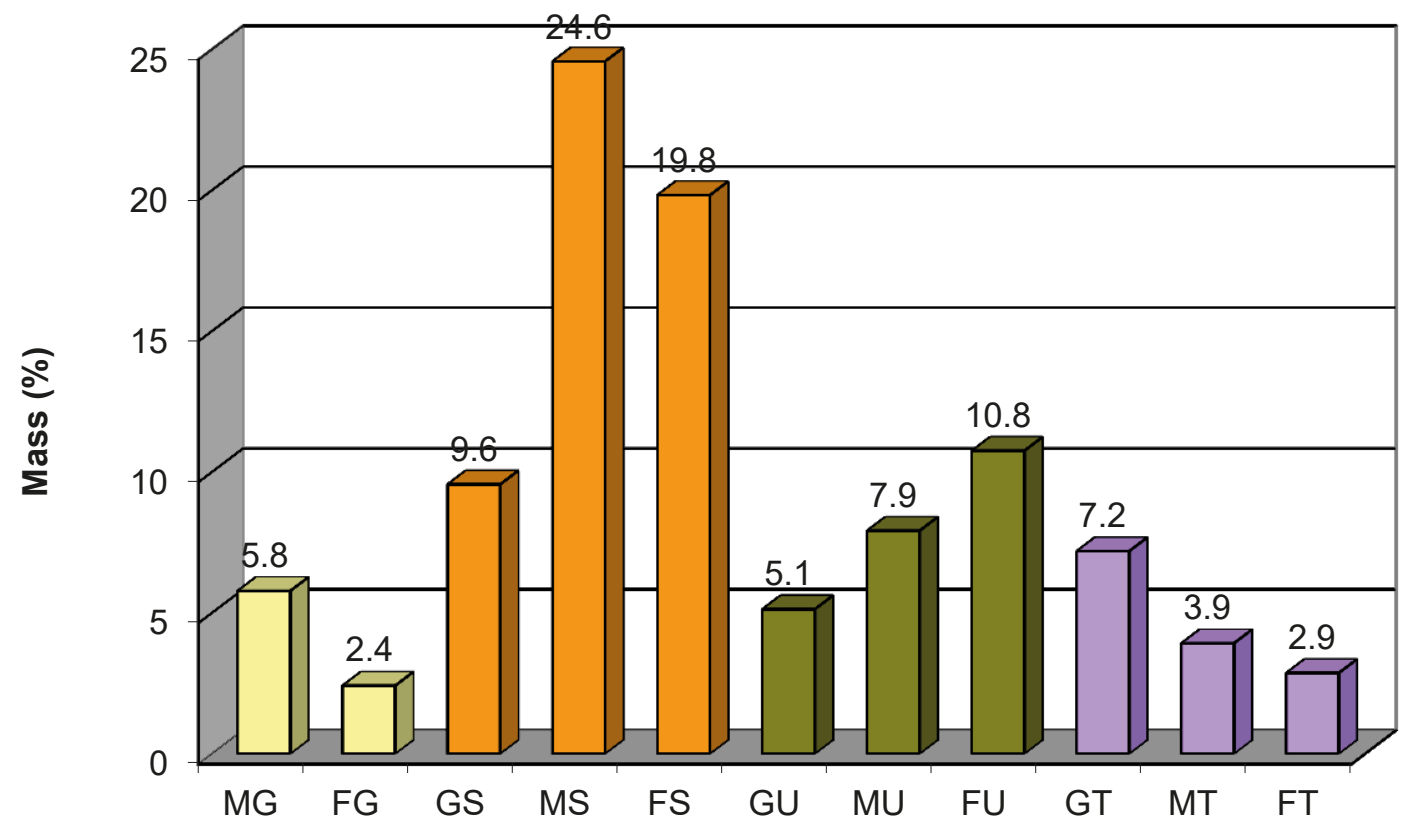

GSD 13.2 Nyarma. Sample 8517. Temple IV. Interior wall plaster. Ground layer.

Top: Cumulative sum. Bottom: Grain size classes. 
11761 - Nyarma. Temple V

Interior wall plaster

\section{Cumulative sum}

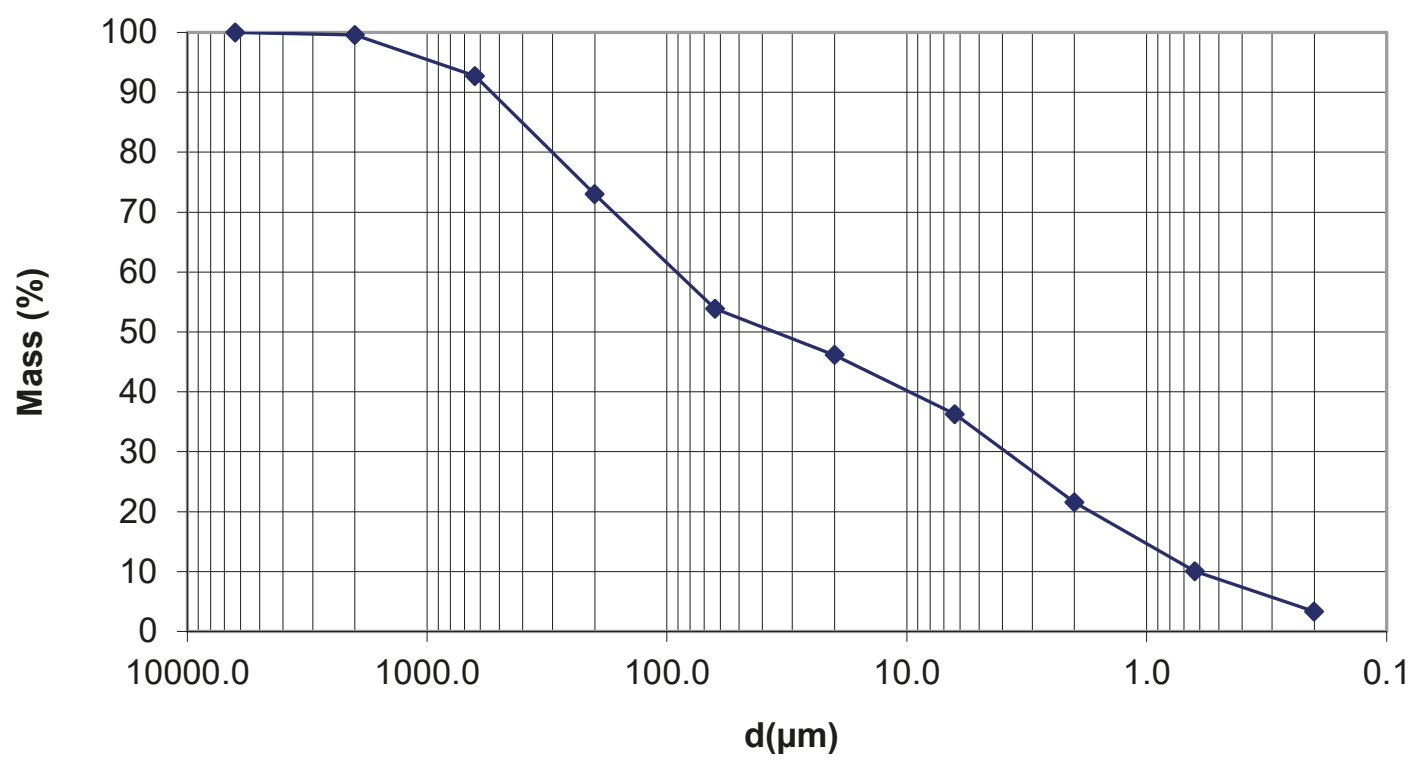

Grain size classes

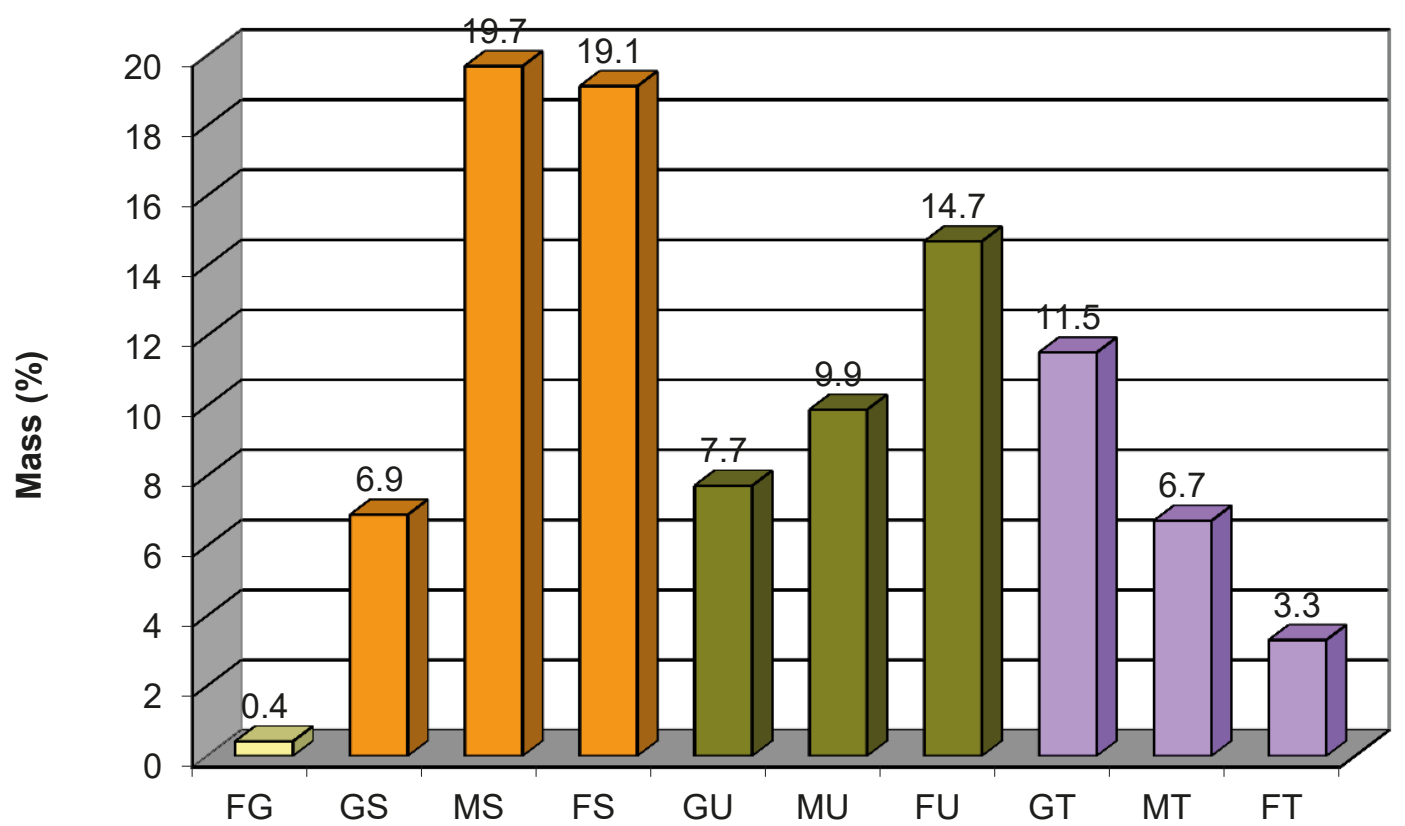

GSD 14.2 Nyarma. Sample 11761. Temple V. Interior wall plaster. Top: Cumulative sum. Bottom: Grain size classes. 


\section{2 - Stupa \\ Interior wall plaster. Ground layer}

\section{Cumulative sum}

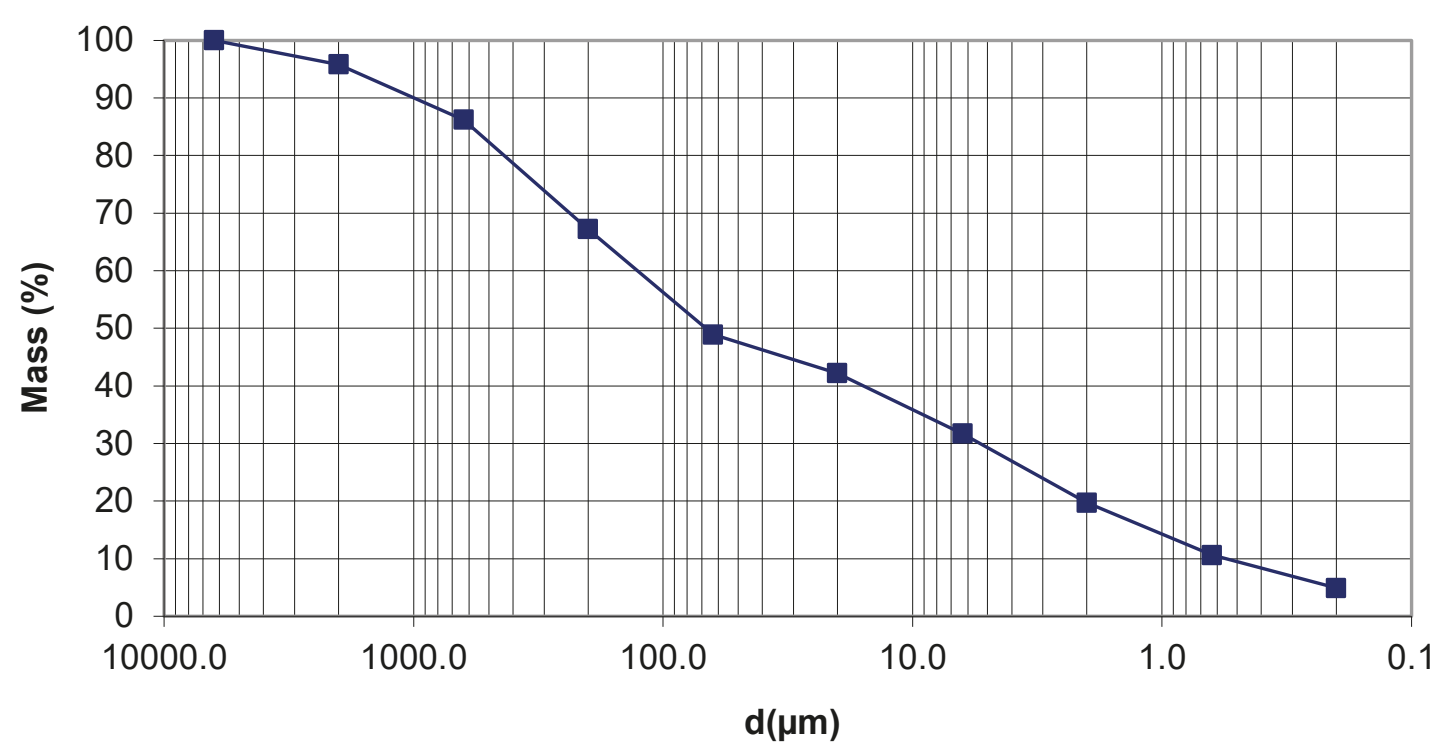

Grain size classes

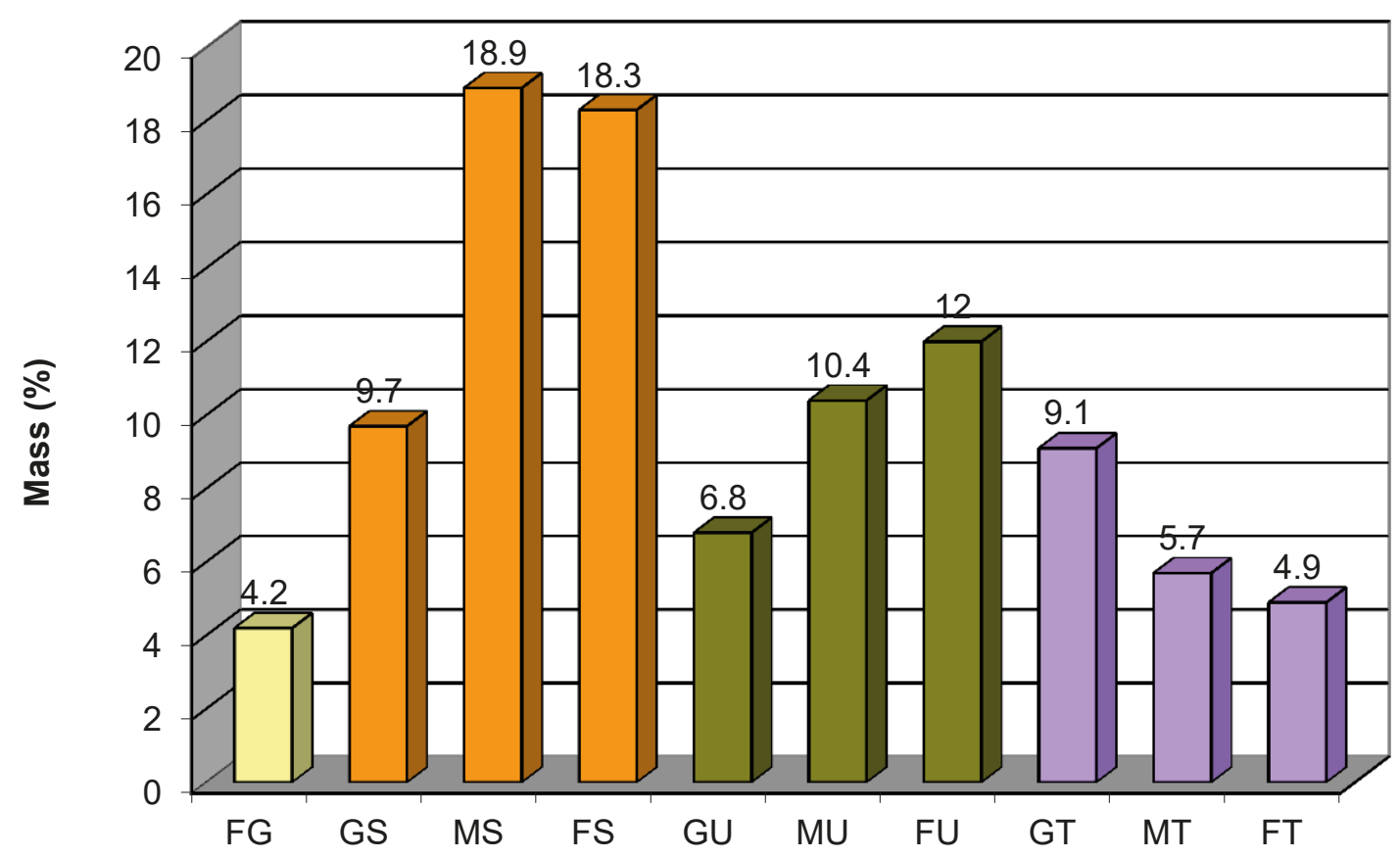

GSD 15.2 Nyarma. Sample 8532. Stupa. Interior wall plaster. Ground layer. Top: Cumulative sum. Bottom: Grain size classes. 

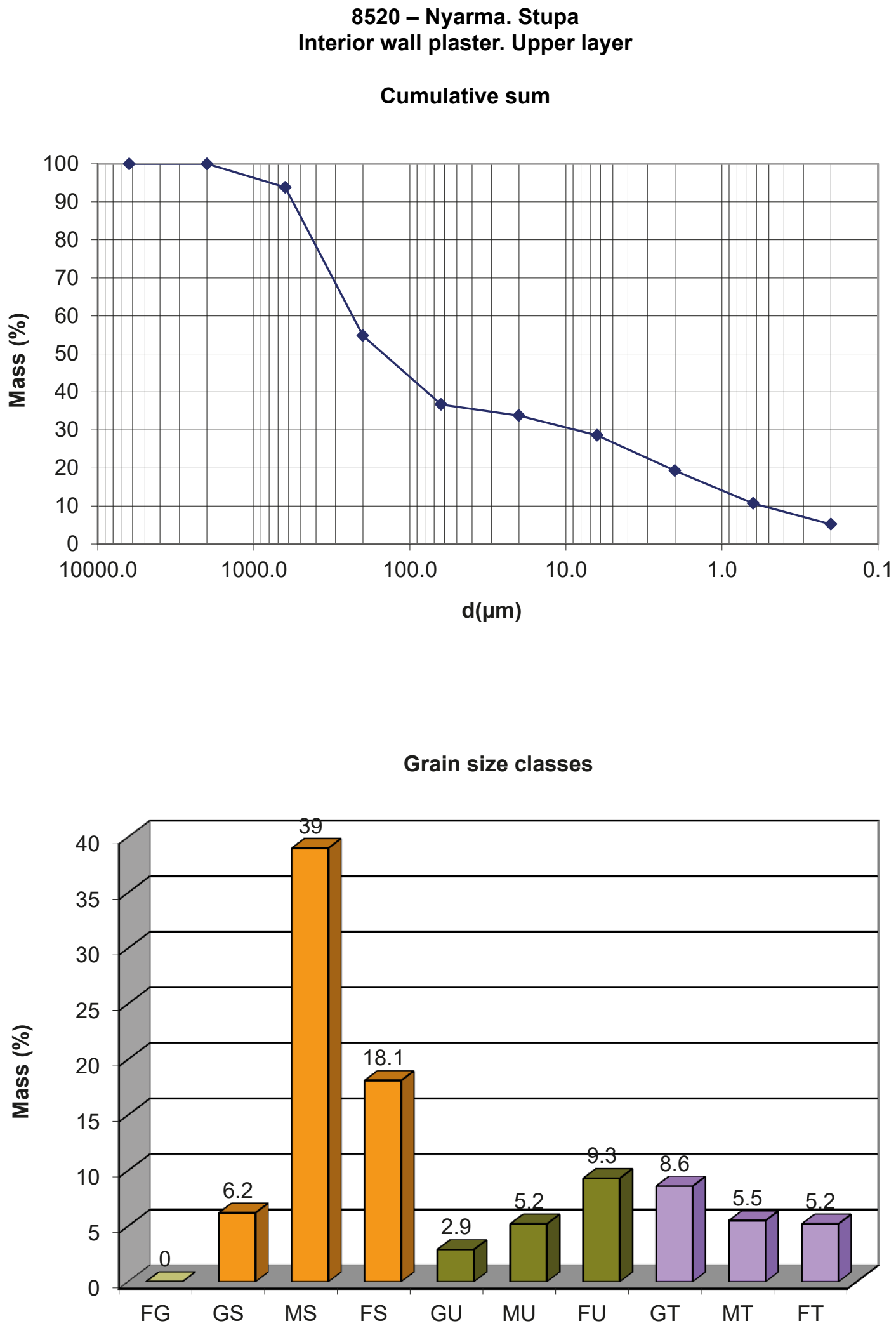

GSD 16.2 Nyarma. Sample 8520. Stupa. Interior wall plaster.

Top: Cumulative sum. Bottom: Grain size classes. 


\section{2 - Nyarma. Temple I. Aureole Ground layer}

\section{Cumulative sum}

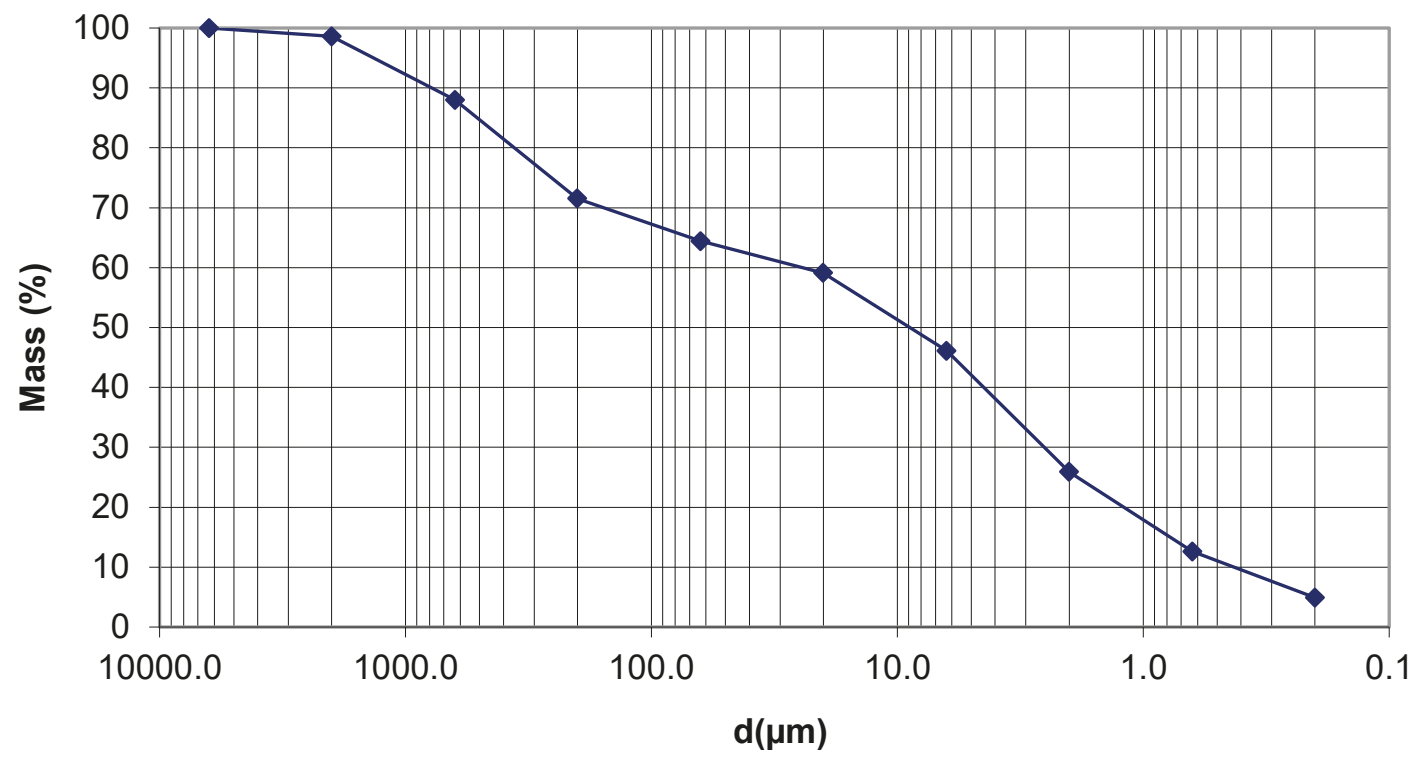

Grain size classes

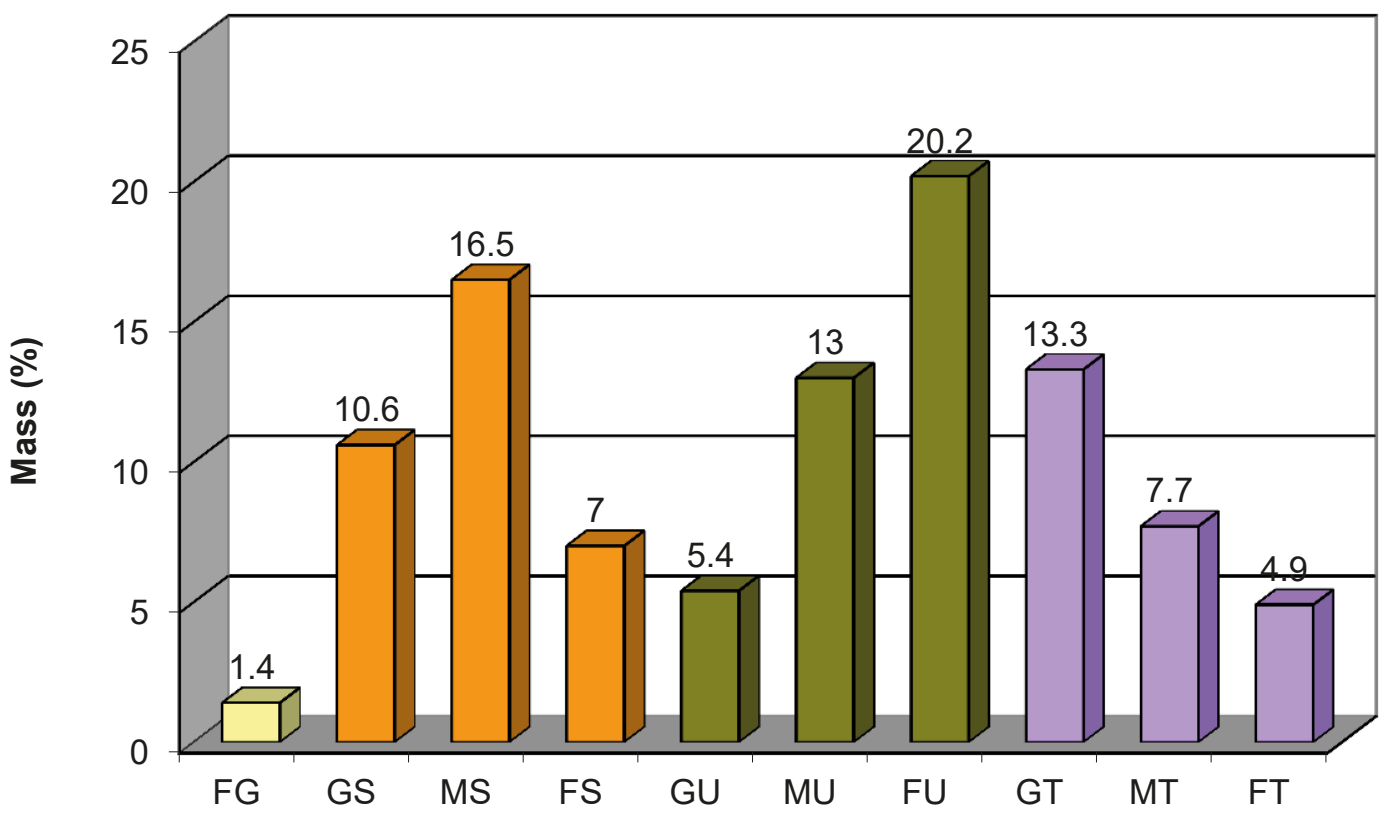

GSD 17.2 Nyarma. Temple I. Sample 8522. Aureole. Ground layer.

Top: Cumulative sum. Bottom: Grain size classes. 


\section{6 - Nyarma. Temple I. Aureole \\ Upper layer}

\section{Cumulative sum}

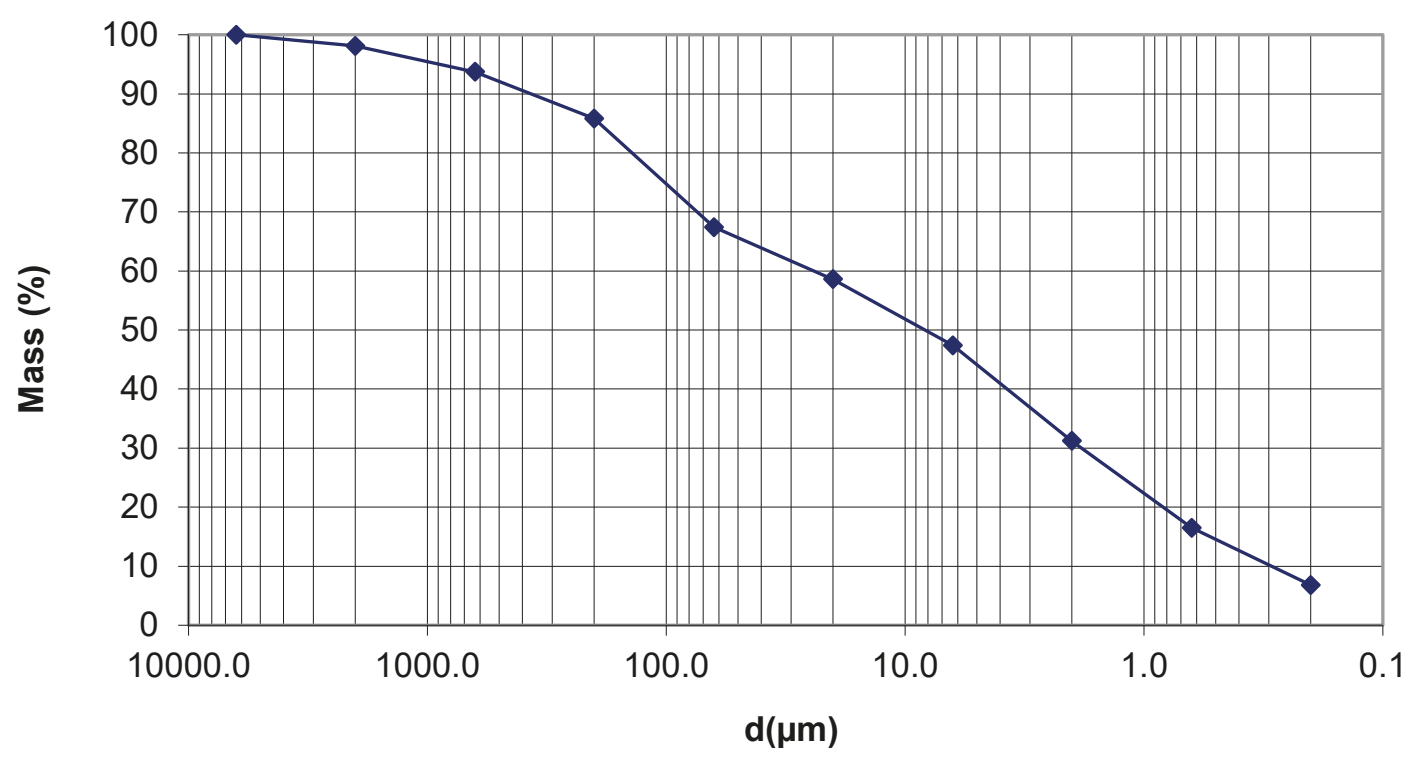

Grain size classes

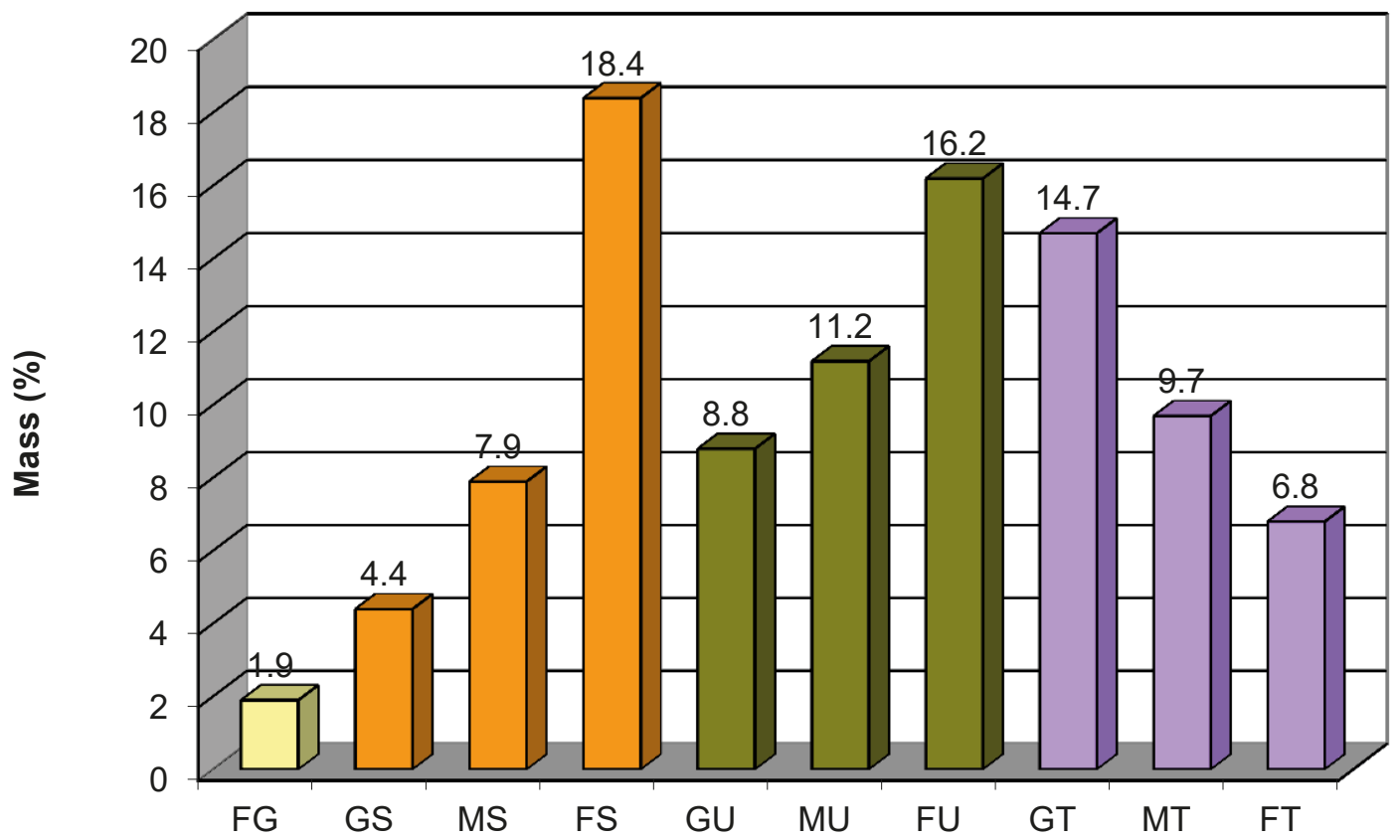

GSD 18.2 Nyarma. Sample 8516. Temple I. Aureole. Upper layer.

Top: Cumulative sum. Bottom: Grain size classes. 


\section{5 - Stupa}

Wall frieze

\section{Cumulative sum}

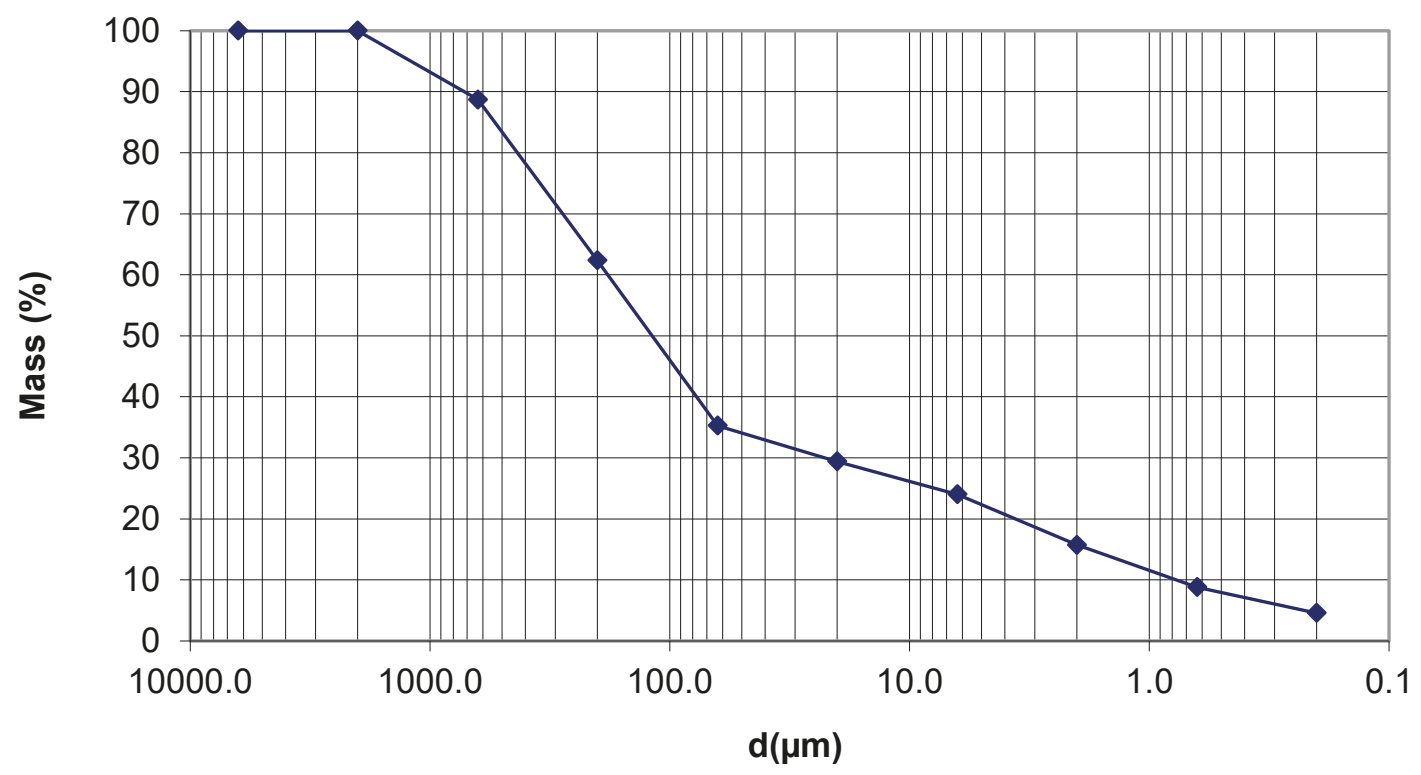

Grain size classes

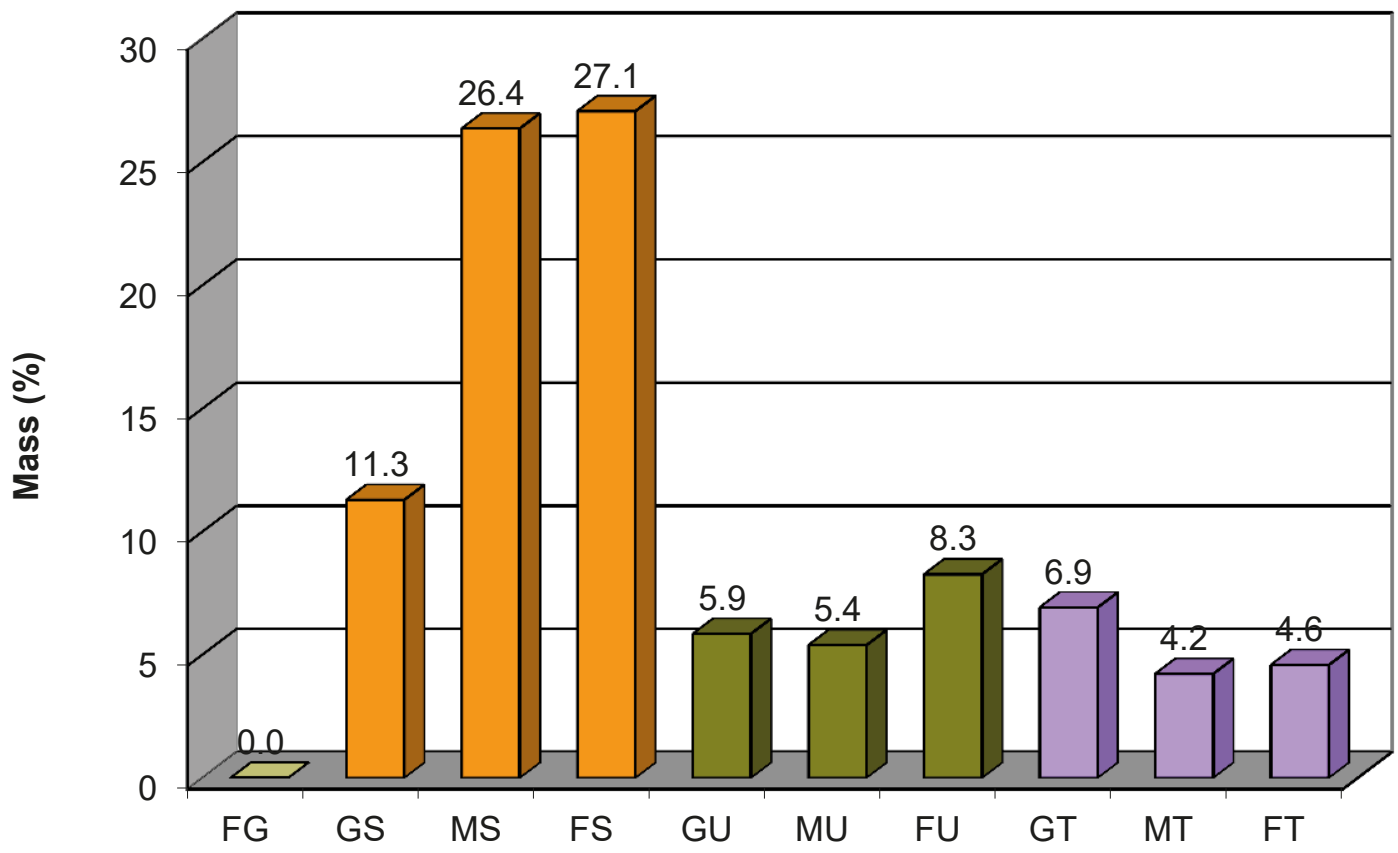

GSD 19.2 Nyarma. Sample 15405. Stupa. Broken piece of a wall frieze.

Top: Cumulative sum. Bottom: Grain size classes. 


\section{4 - Nyarma. Temple I. Cella north wall \\ Exterior plaster}

\section{Cumulative sum}

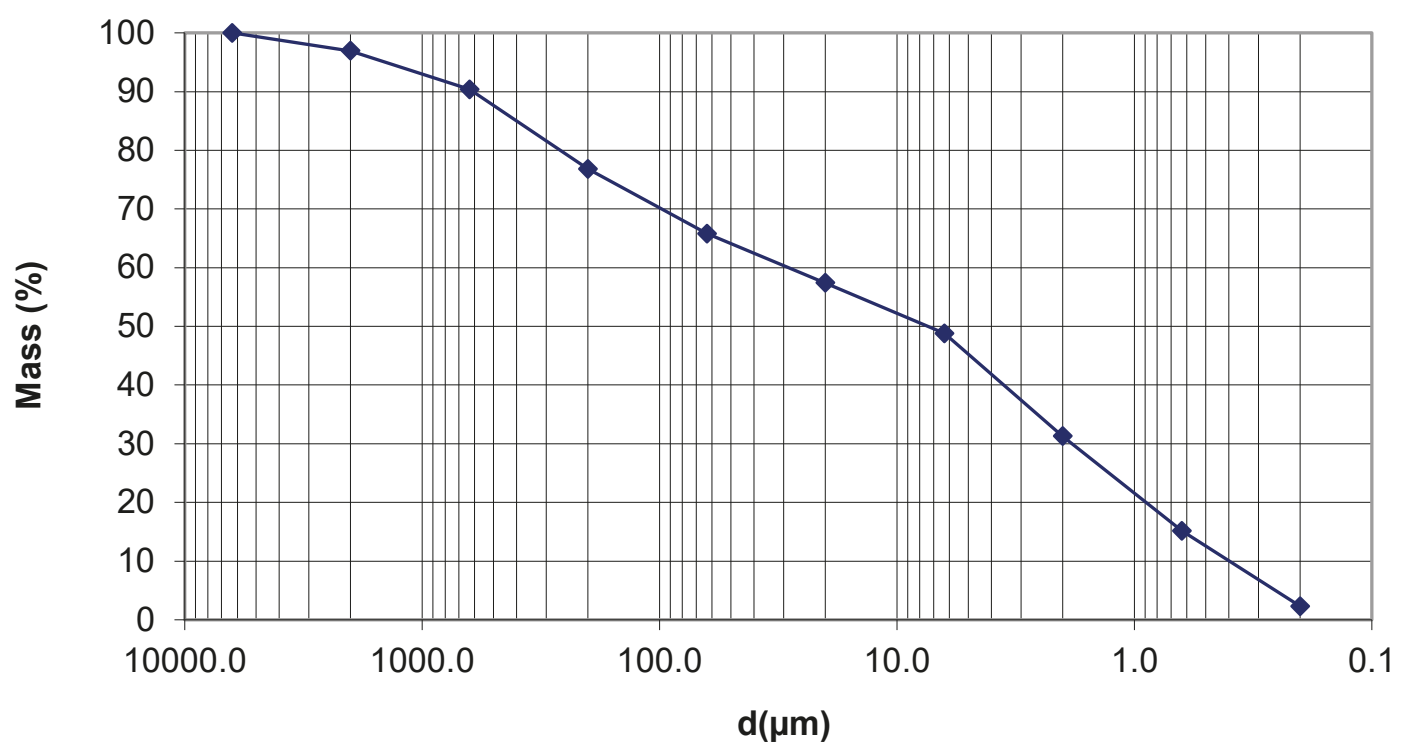

Grain size classes

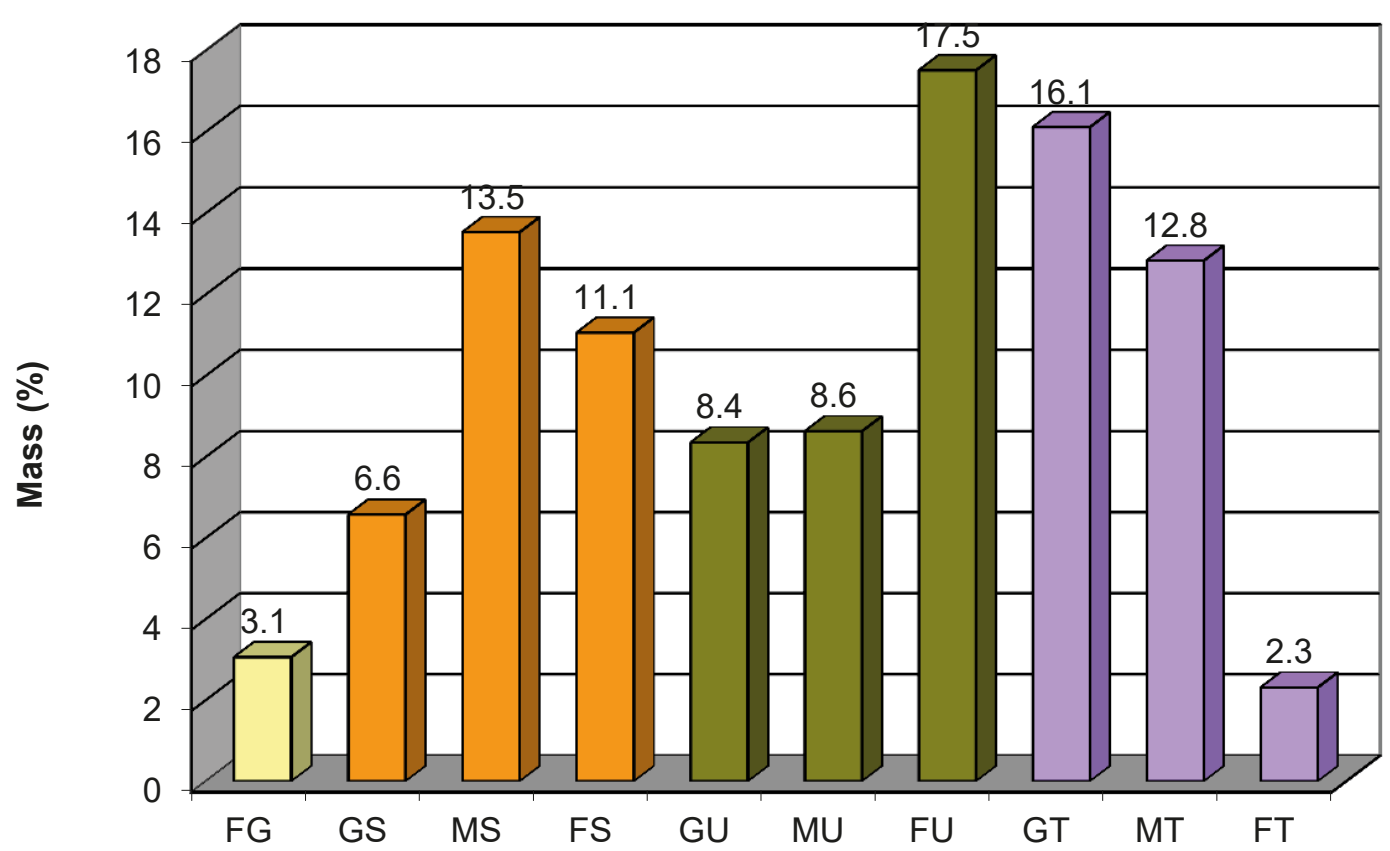

GSD 20.2 Nyarma. Sample 11914. Temple I. Cella north wall. Exterior wall plaster. Top: Cumulative sum. Bottom: Grain size classes. 


\section{2 - Nyarma. Temple I. Assembly hall south wall}

Exterior plaster

\section{Cumulative sum}

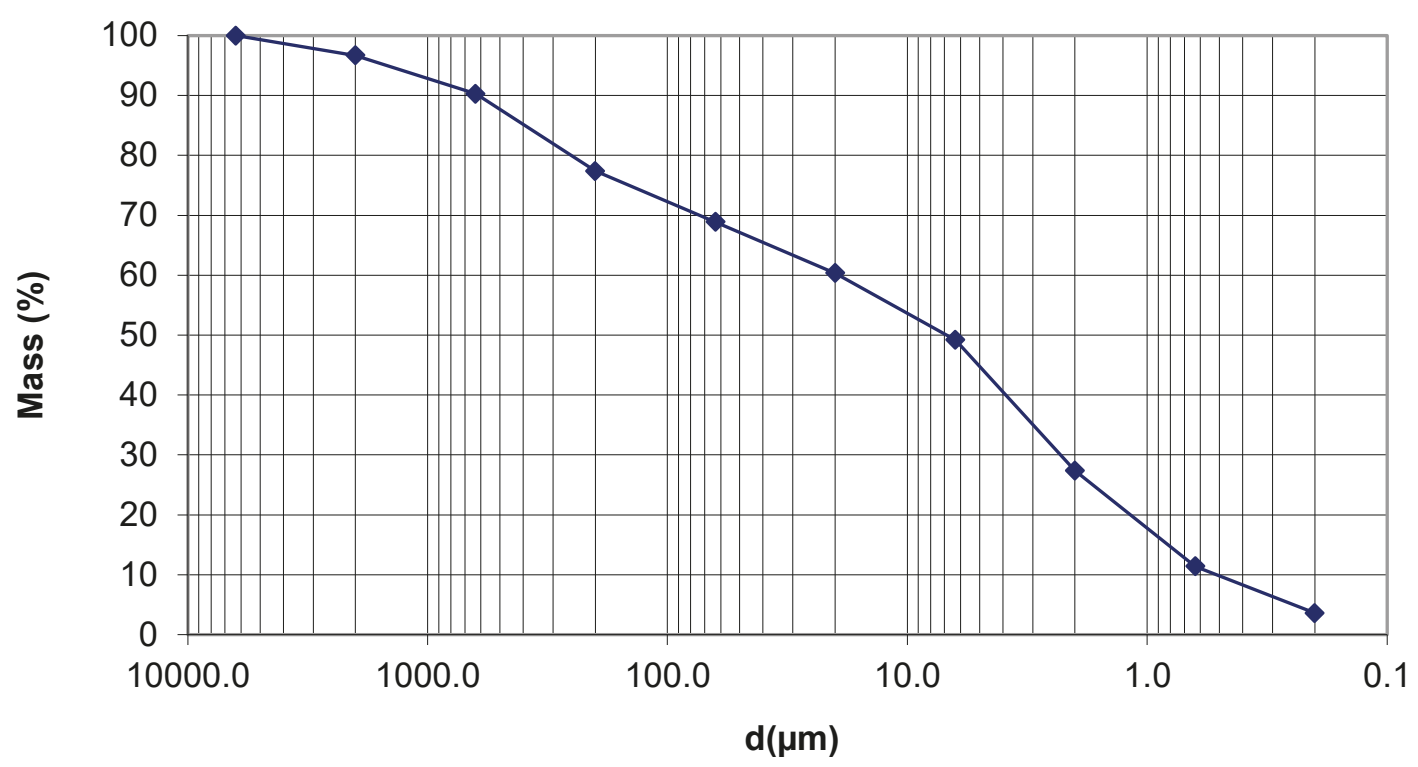

Grain size classes

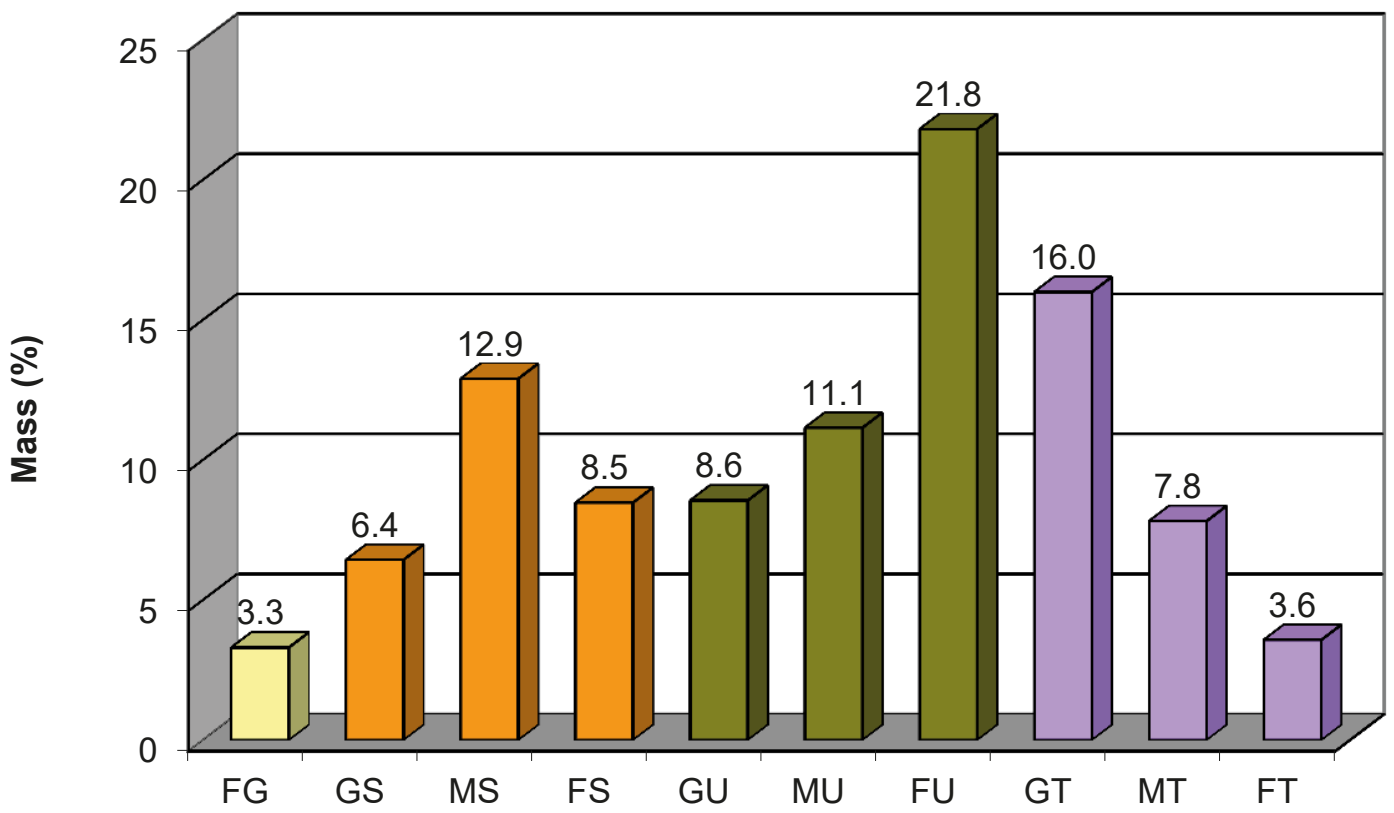

GSD 21.2 Nyarma. Sample 11762. Temple I. Assembly hall south wall. Exterior wall plaster. Top: Cumulative sum. Bottom: Grain size classes. 


\section{8 - Nyarma. Temple IIIb Exterior plaster}

Cumulative sum

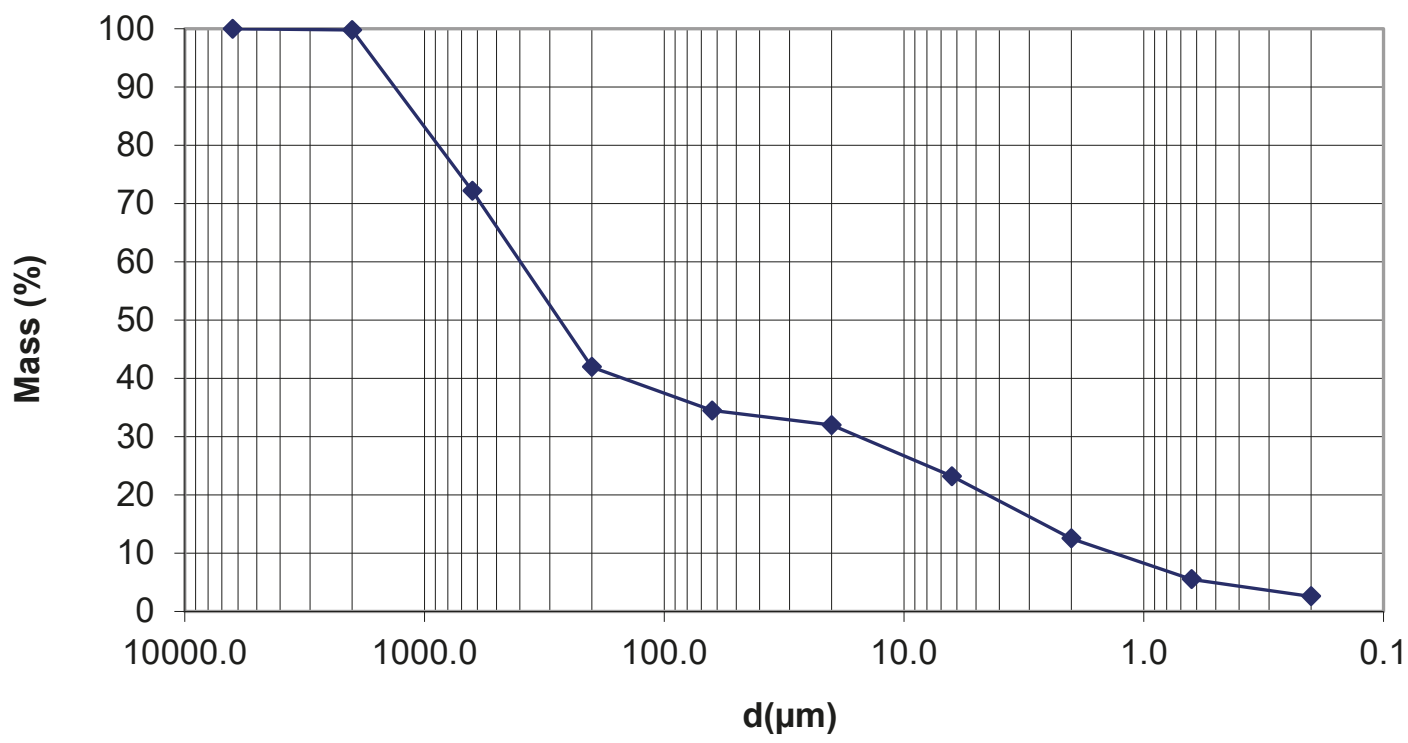

Grain size classes

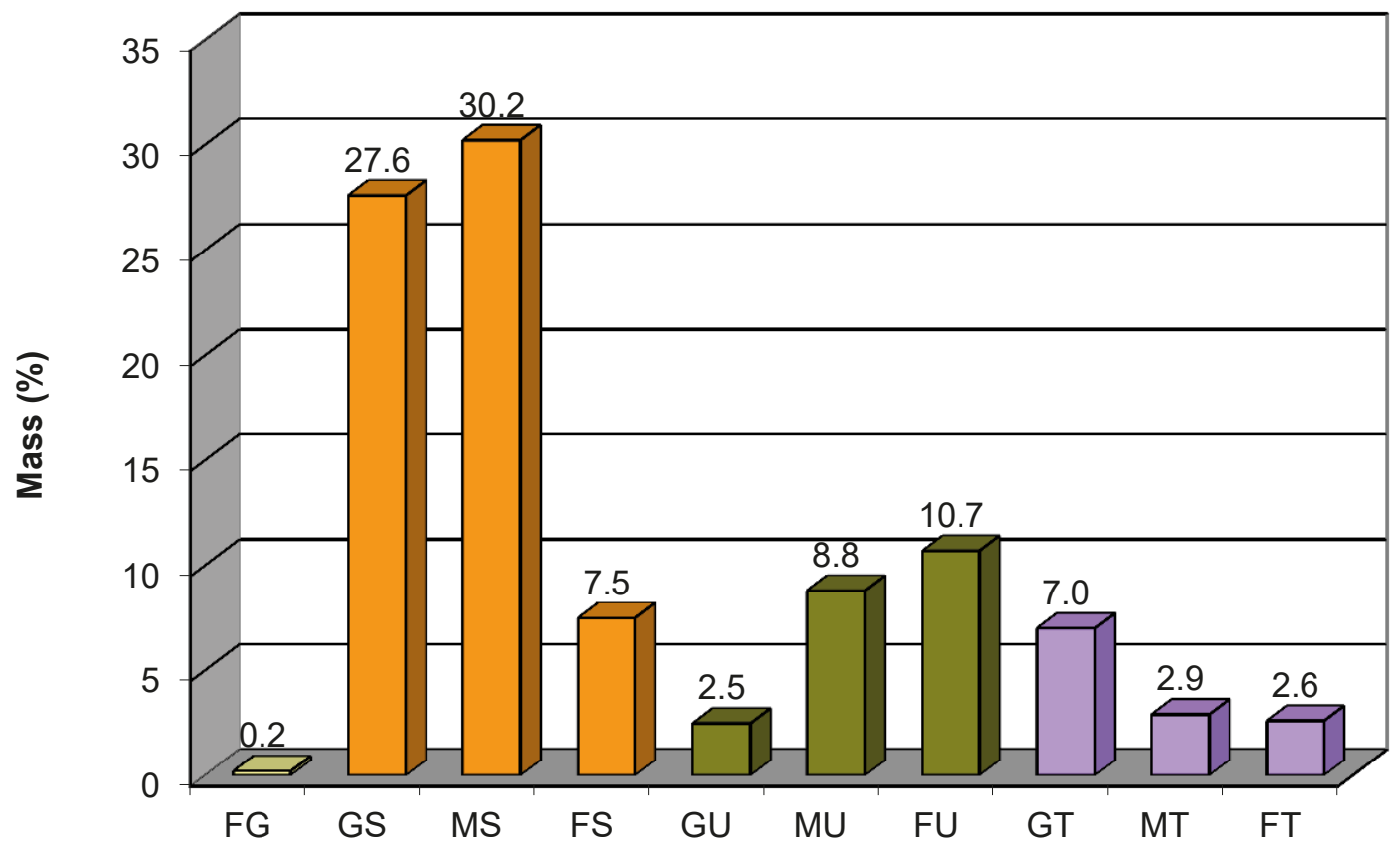

GSD 22.2 Nyarma. Sample 15408. Temple IIIb. Exterior wall plaster.

Top: Cumulative sum. Bottom: Grain size classes. 


\section{9 - Nyarma. Temple IV \\ Exterior plaster. Upper layer}

Cumulative sum

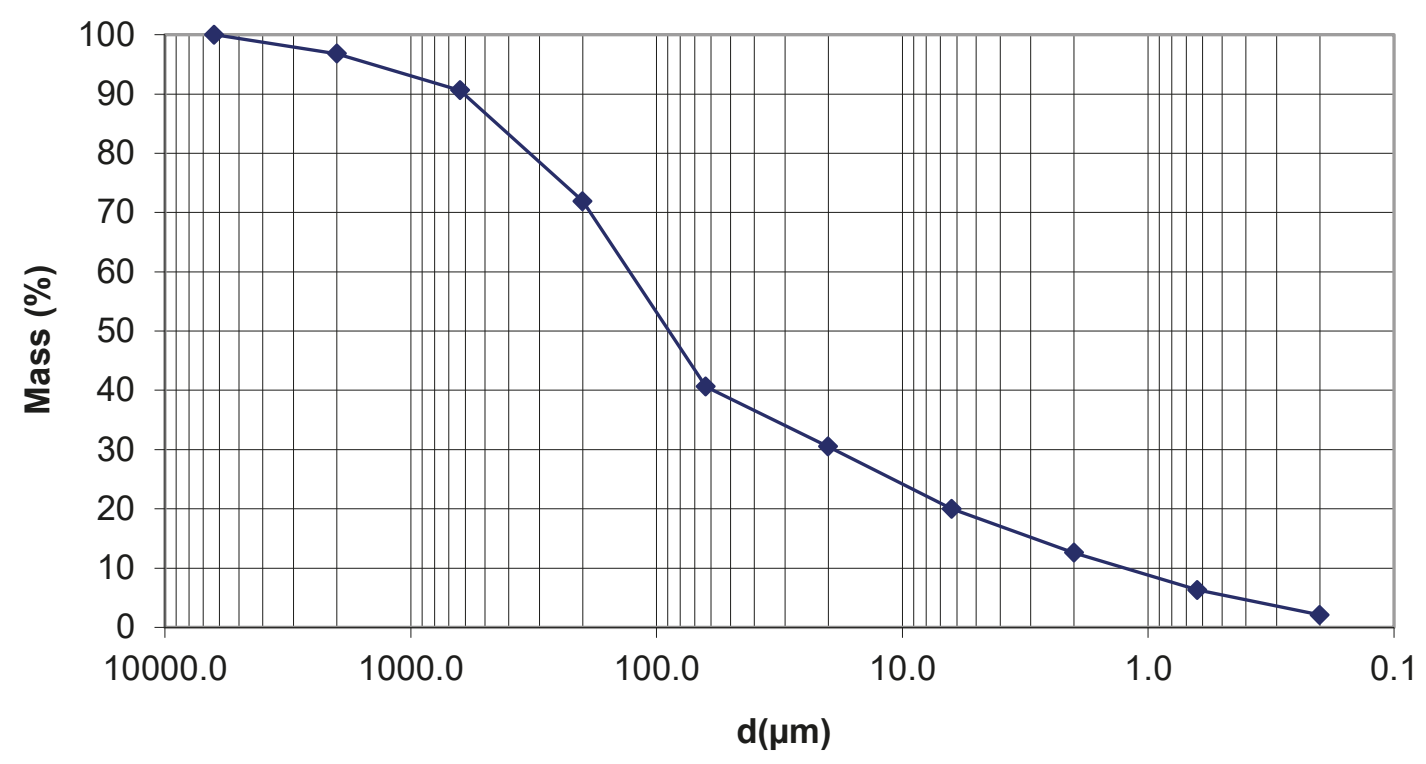

Grain size classes

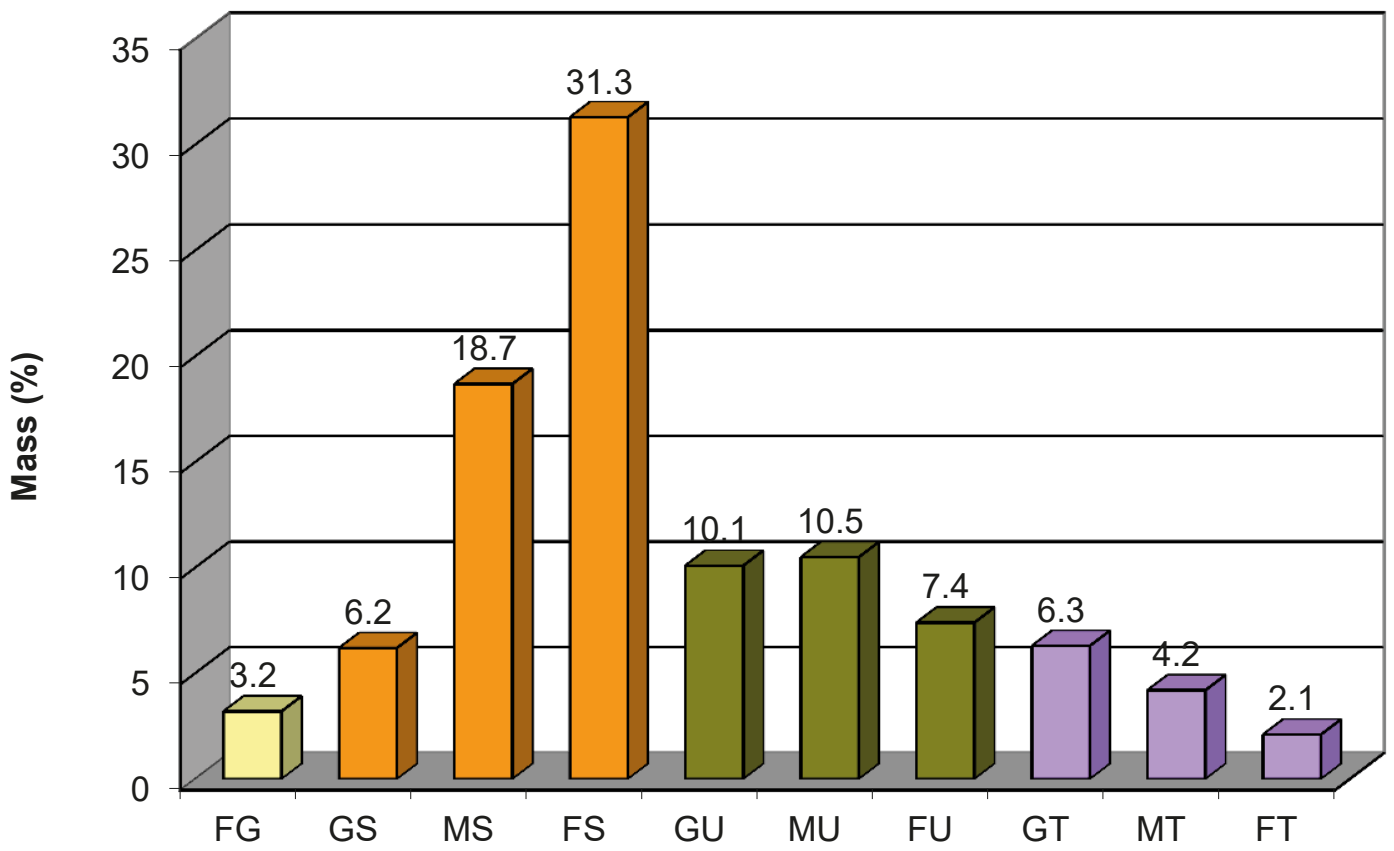

GSD 23.2 Nyarma. Sample 8519. Temple IV. Exterior wall plaster. Upper layer.

Top: Cumulative sum. Bottom: Grain size classes. 


\section{7 - Nyarma. Temple I. Assembly hall Adobe brick}

Cumulative sum

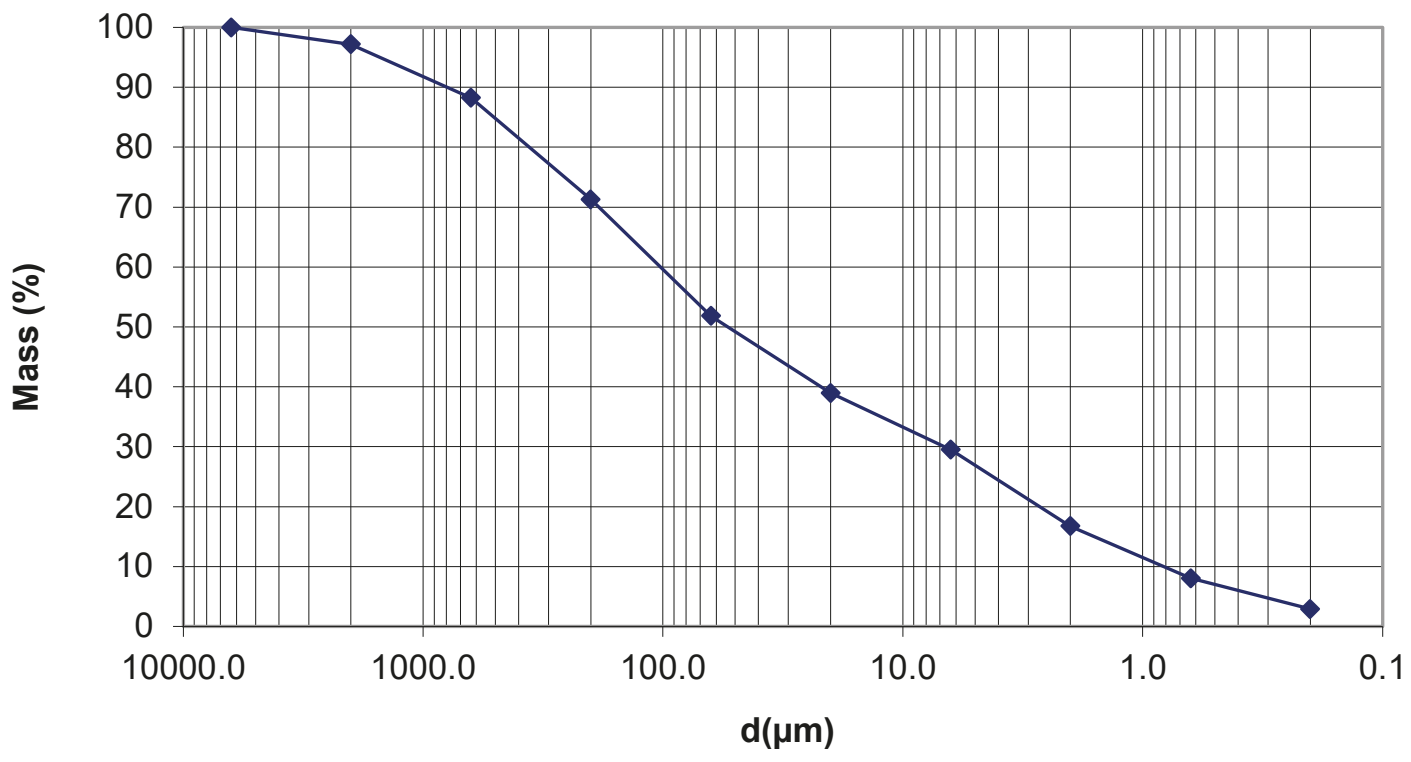

Grain size classes

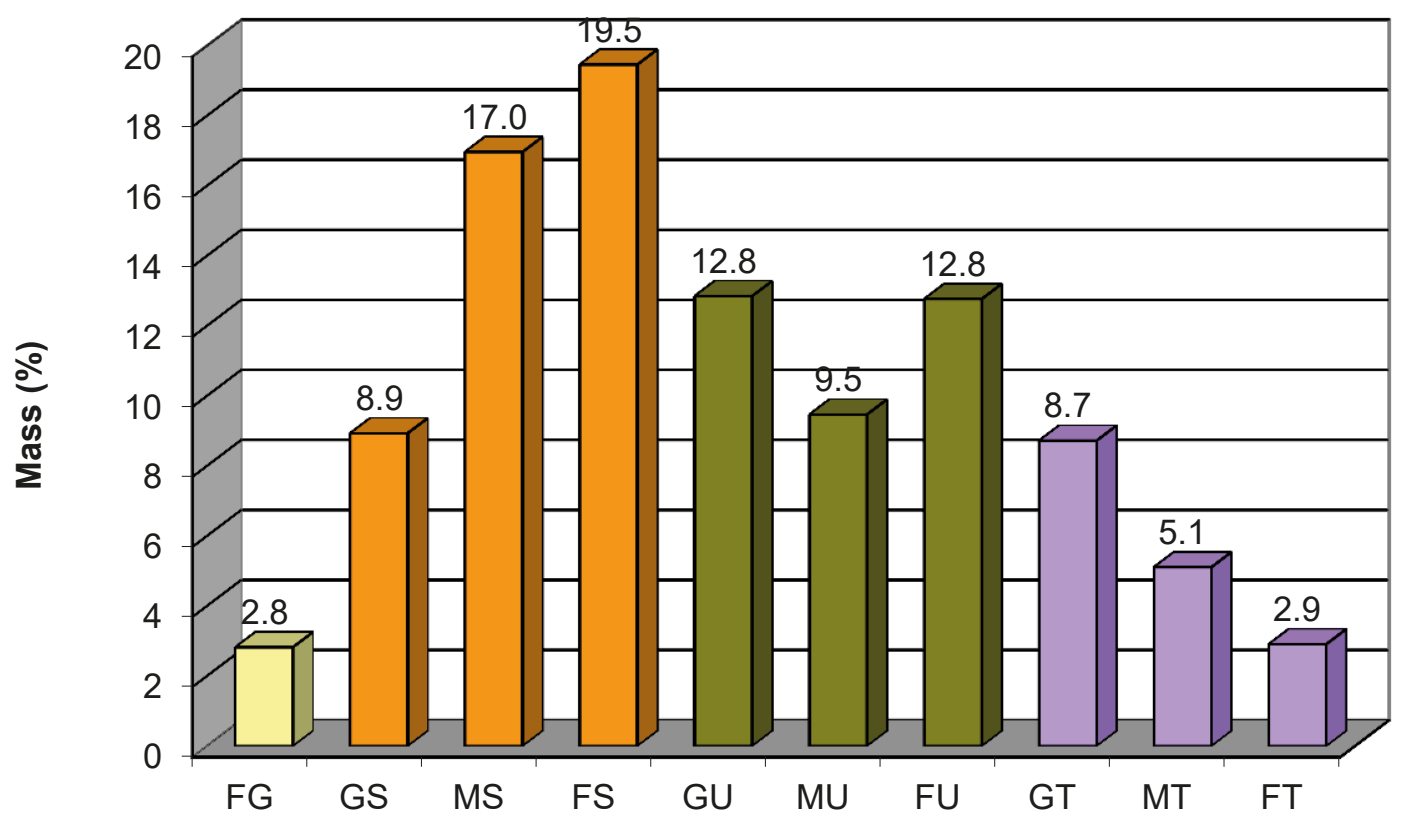

GSD 24.2 Nyarma. Sample 11767. Temple I. Assembly hall. Adobe brick.

Top: Cumulative sum. Bottom: Grain size classes. 


\section{9 - Nyarma. Temple II \\ Adobe brick}

\section{Cumulative sum}

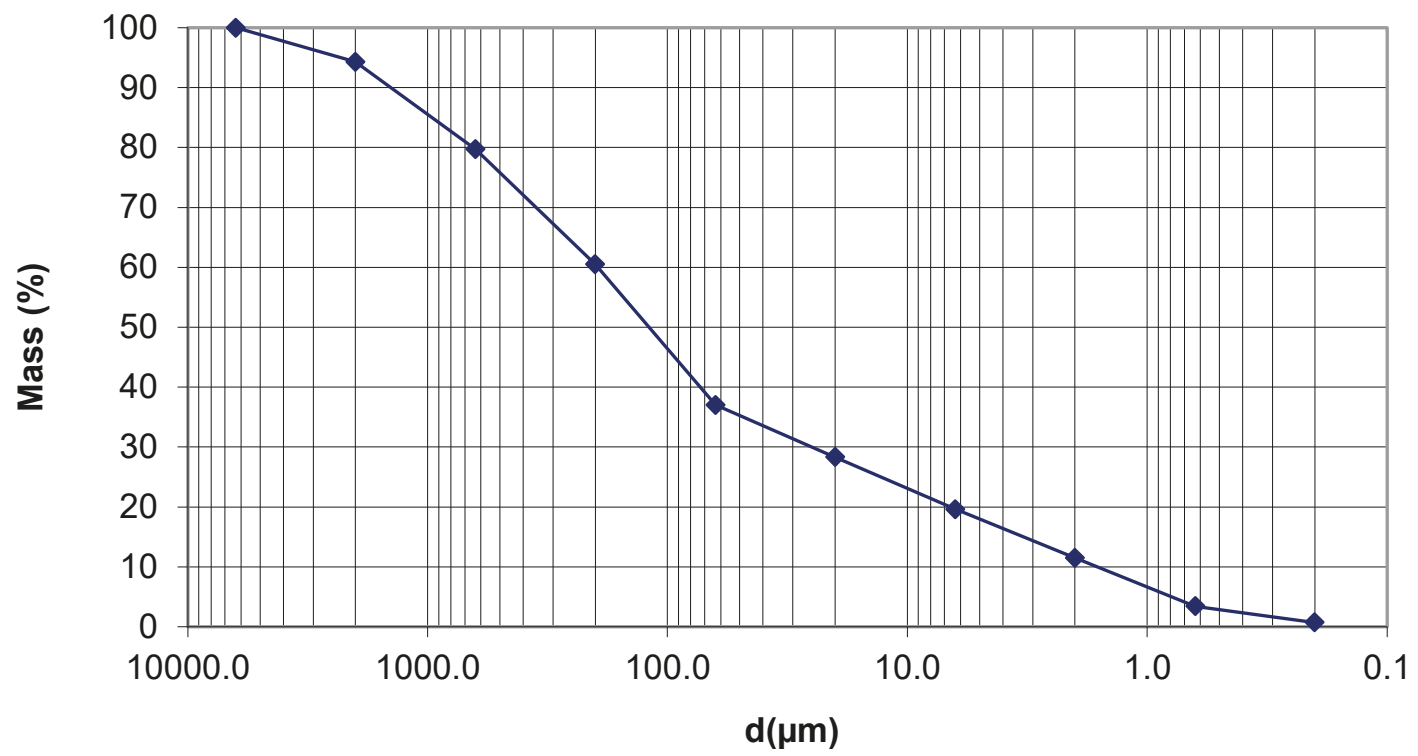

Grain size classes

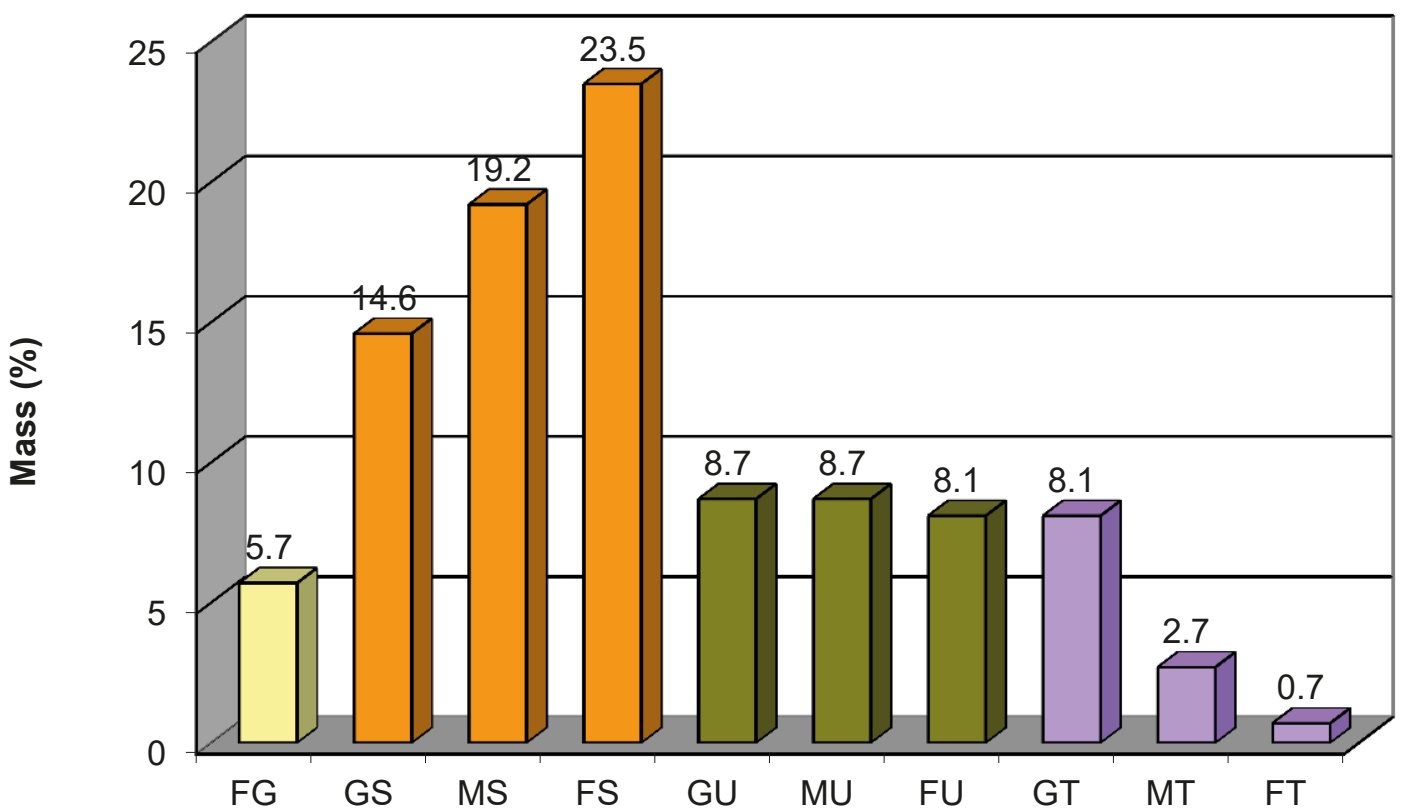

GSD 25.2 Nyarma. Sample 8509. Temple II. Adobe brick.

Top: Cumulative sum. Bottom: Grain size classes. 


\section{0 - Nyarma. Temple II} Adobe brick

\section{Cumulative sum}

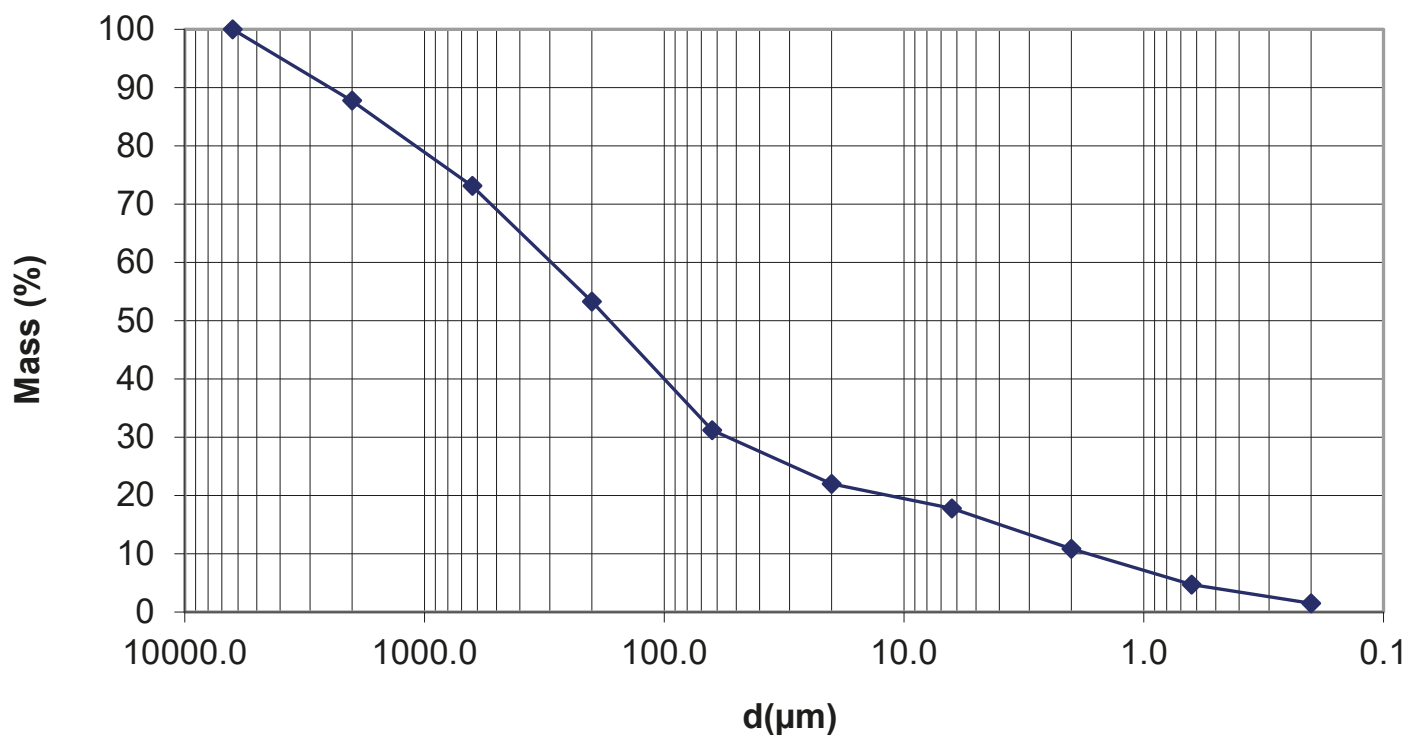

\section{Grain size classes}

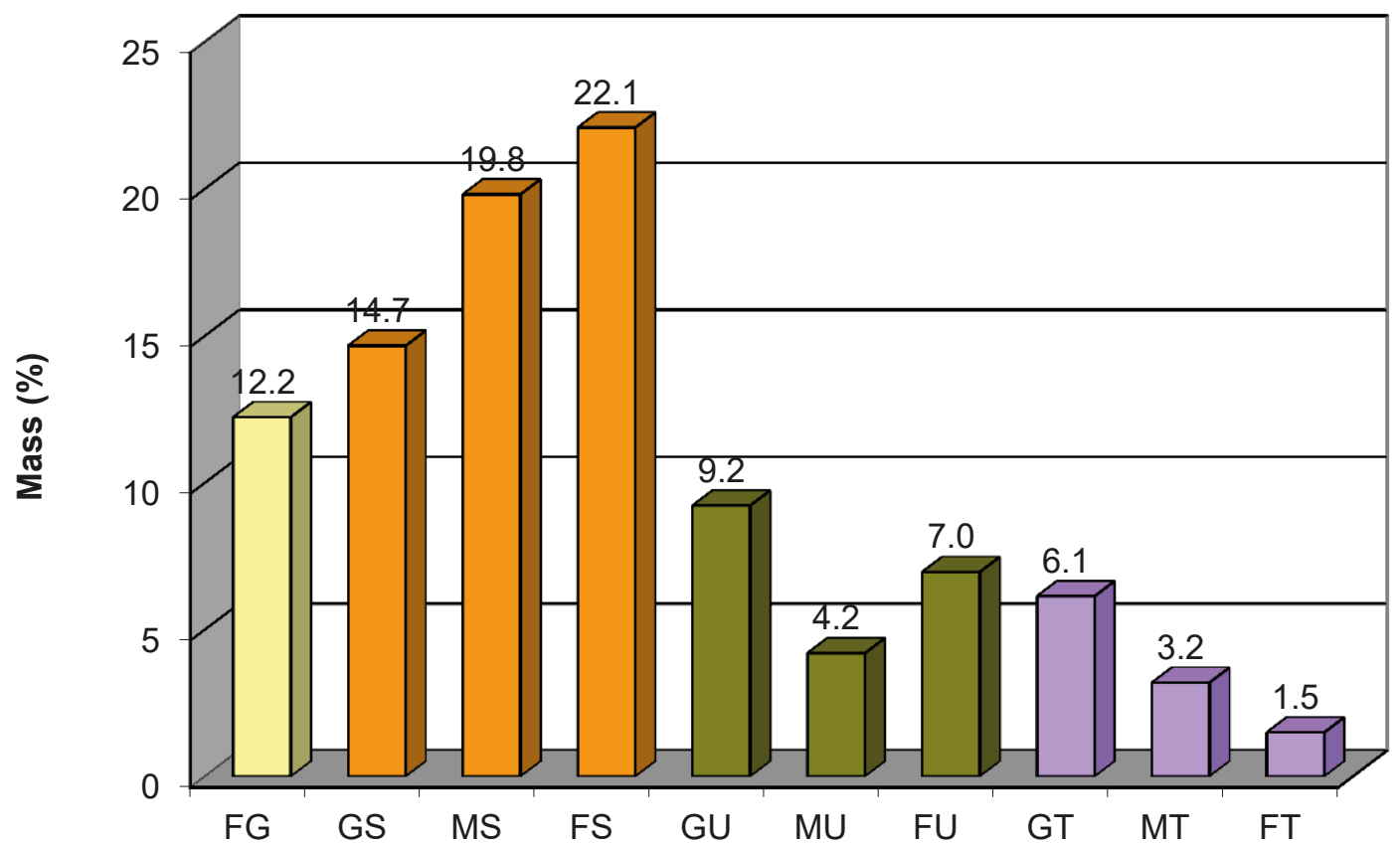

GSD 26.2 Nyarma. Sample 11750. Temple II. Adobe brick.

Top: Cumulative sum. Bottom: Grain size classes. 


\section{6 - Temple Illa \\ Adobe brick}

\section{Cumulative sum}

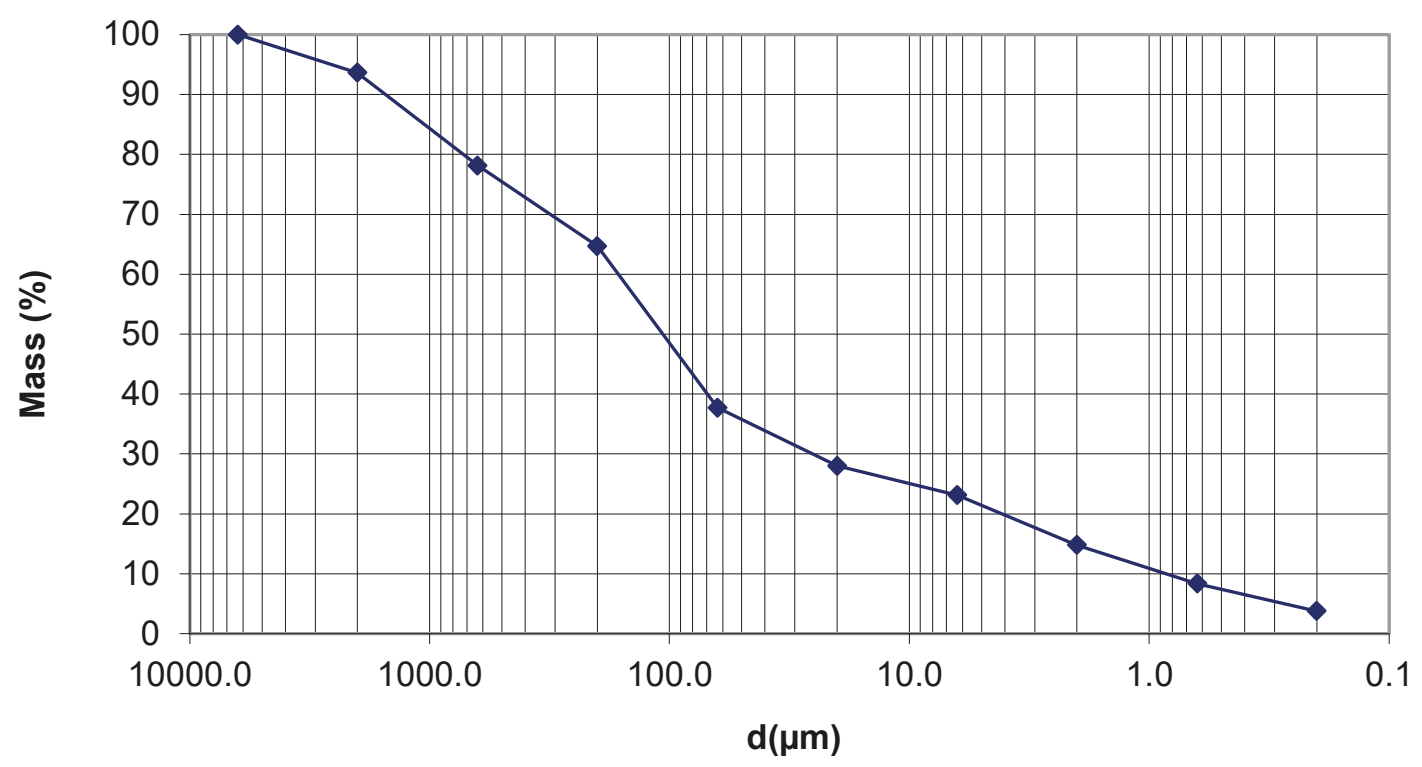

Grain size classes

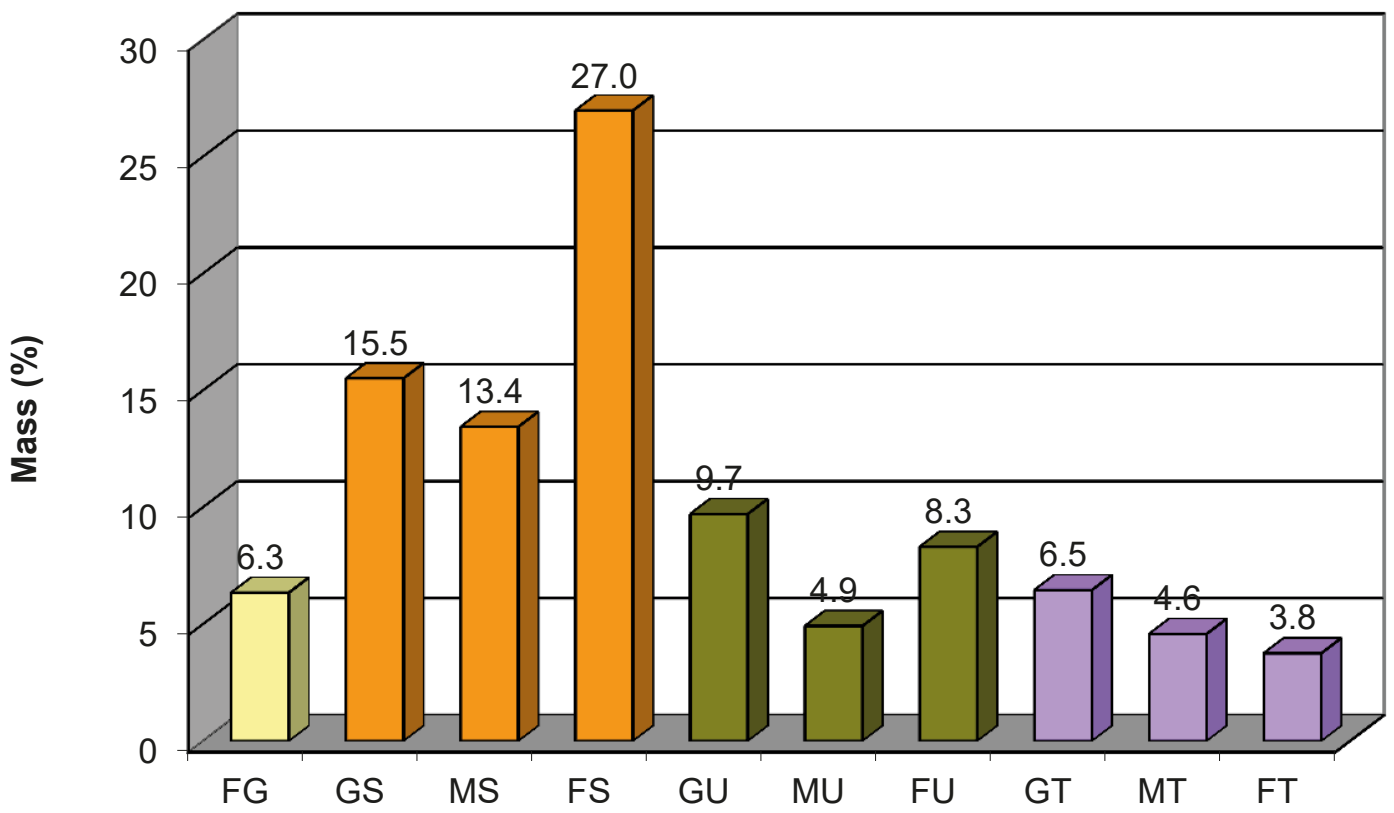

GSD 27.2 Nyarma. Sample 11926. Temple IIIa. Adobe brick.

Top: Cumulative sum. Bottom: Grain size classes. 


\section{4 - Nyarma. Temple IIIb \\ Adobe brick}

\section{Cumulative sum}

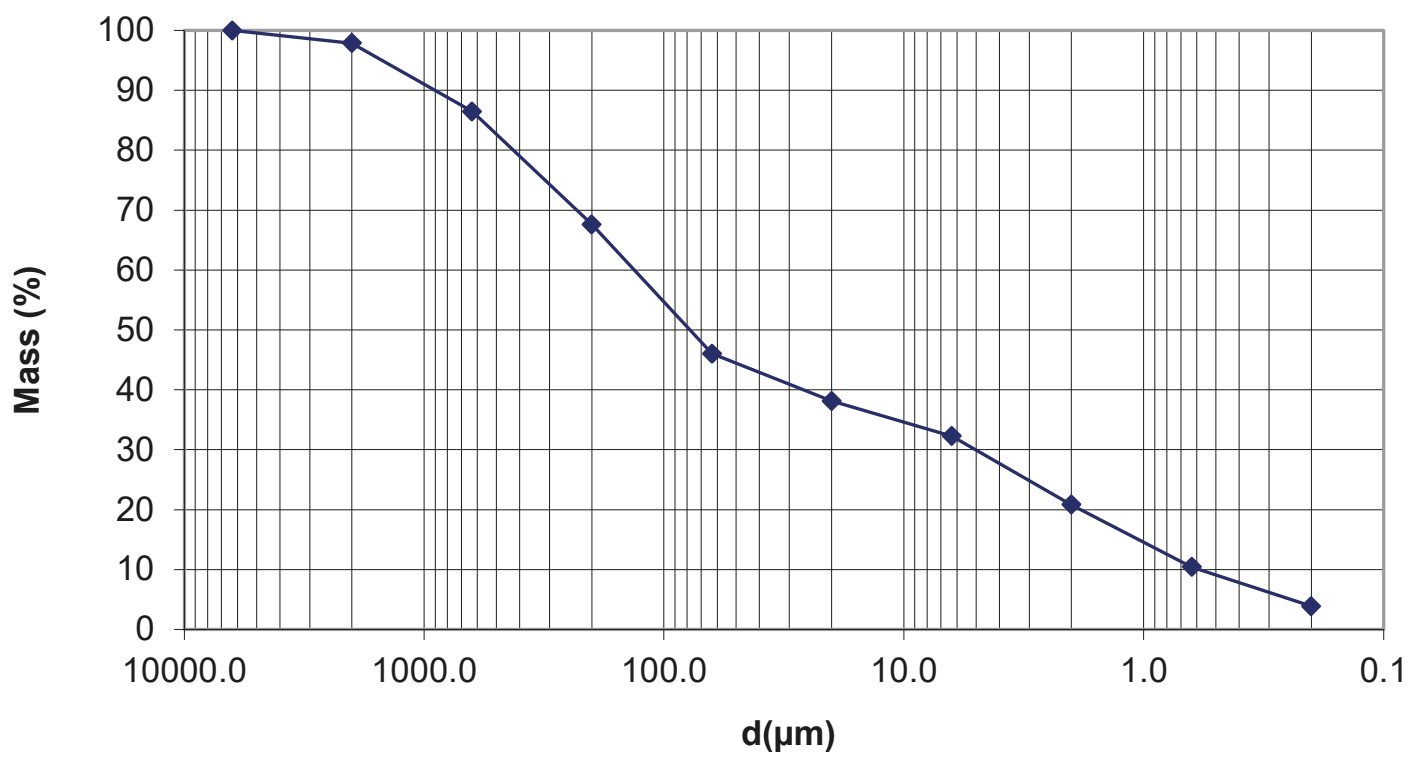

Grain size classes

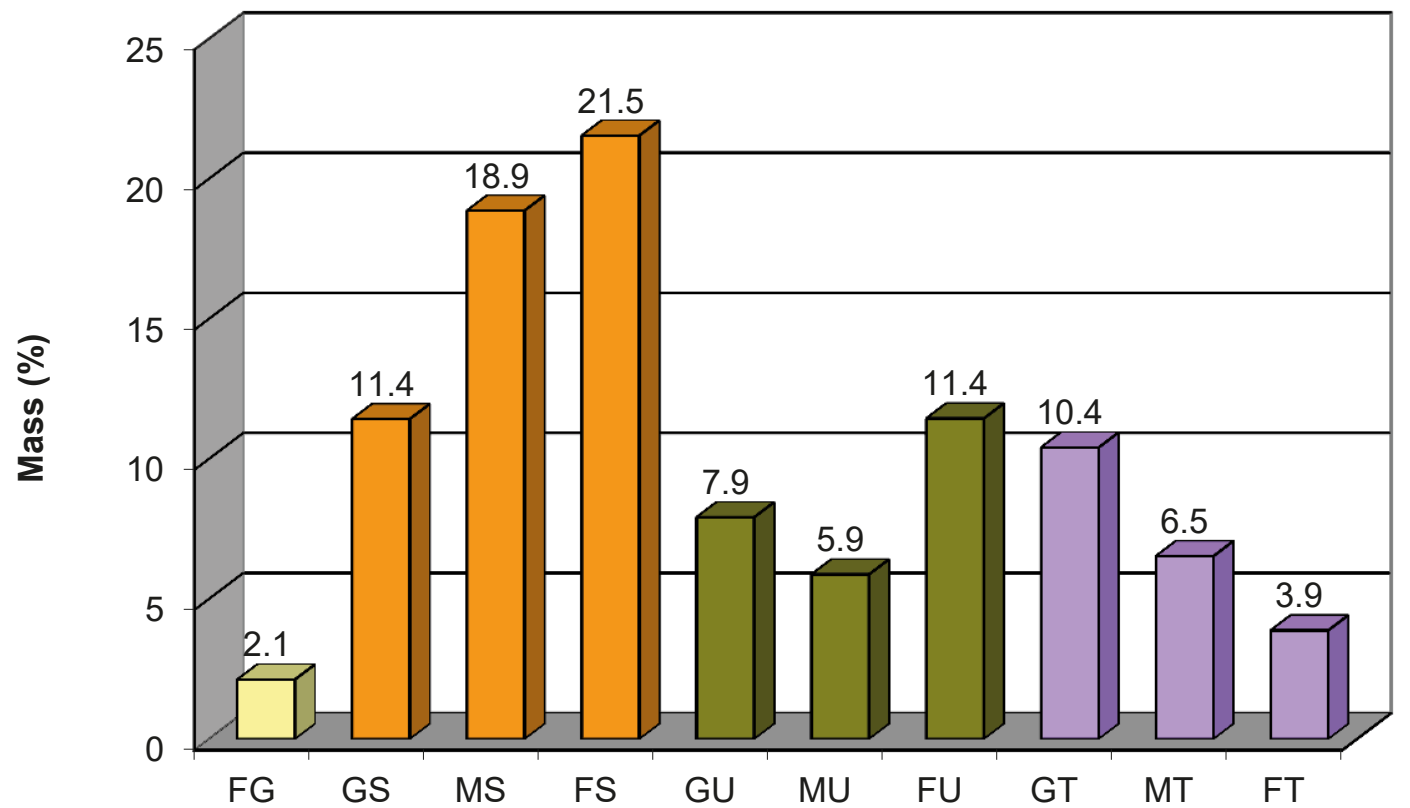

GSD 28.2 Nyarma. Sample 11744. Temple IIIb. Adobe brick.

Top: Cumulative sum. Bottom: Grain size classes. 


\section{3 - Nyarma. Temple IIIb Adobe brick}

\section{Cumulative sum}

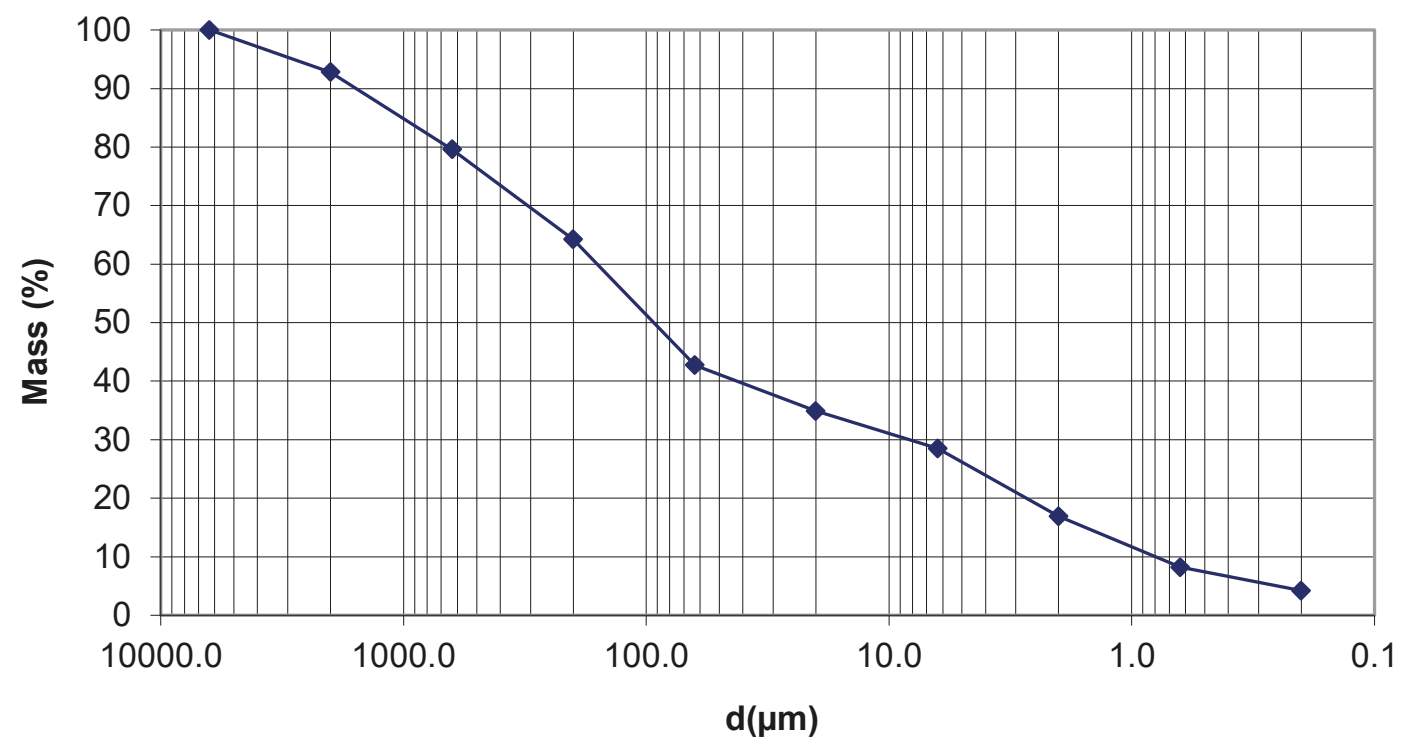

Grain size classes

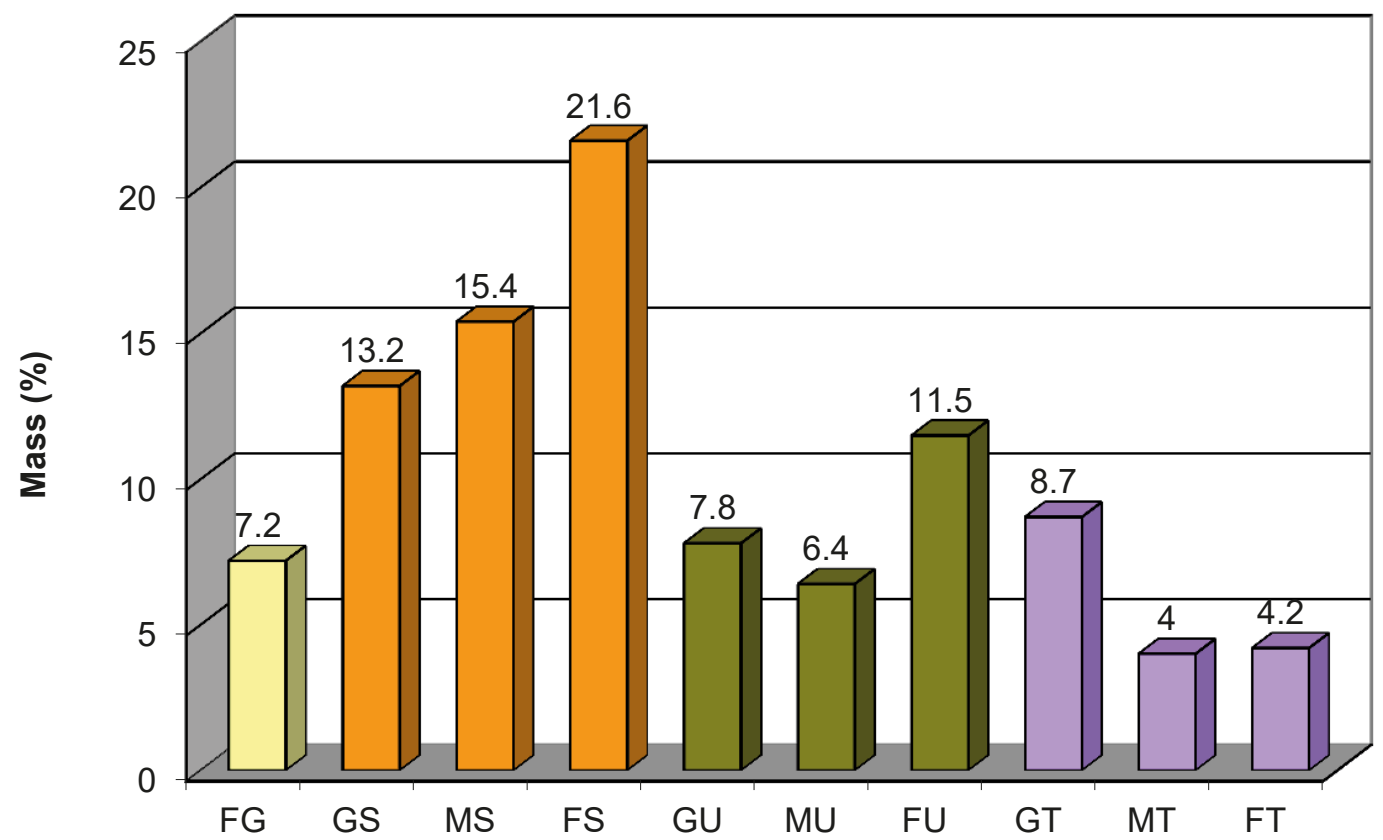

GSD 29.2 Nyarma. Sample 8533. Temple IIIb. Adobe brick.

Top: Cumulative sum. Bottom: Grain size classes. 


\section{7 - Nyarma. Temple IV \\ Adobe brick}

\section{Cumulative sum}

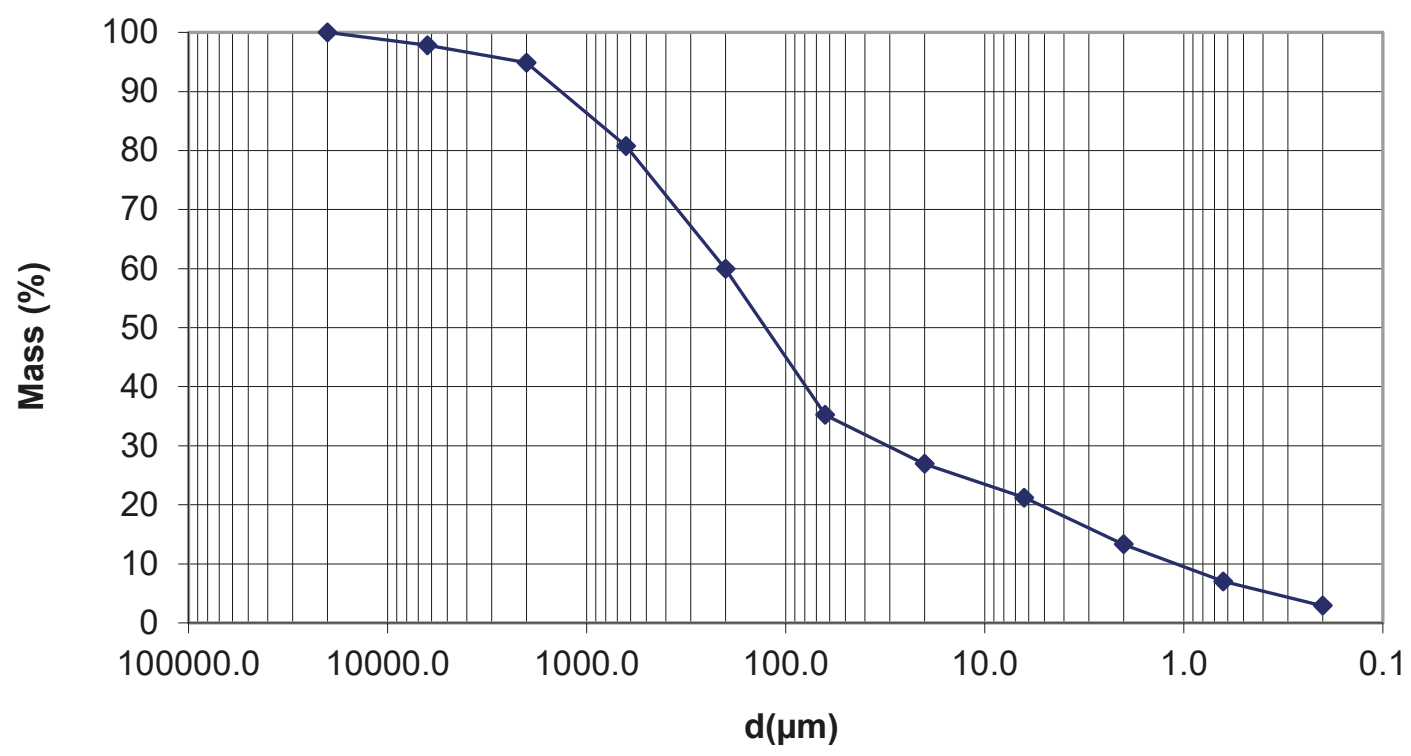

Grain size classes

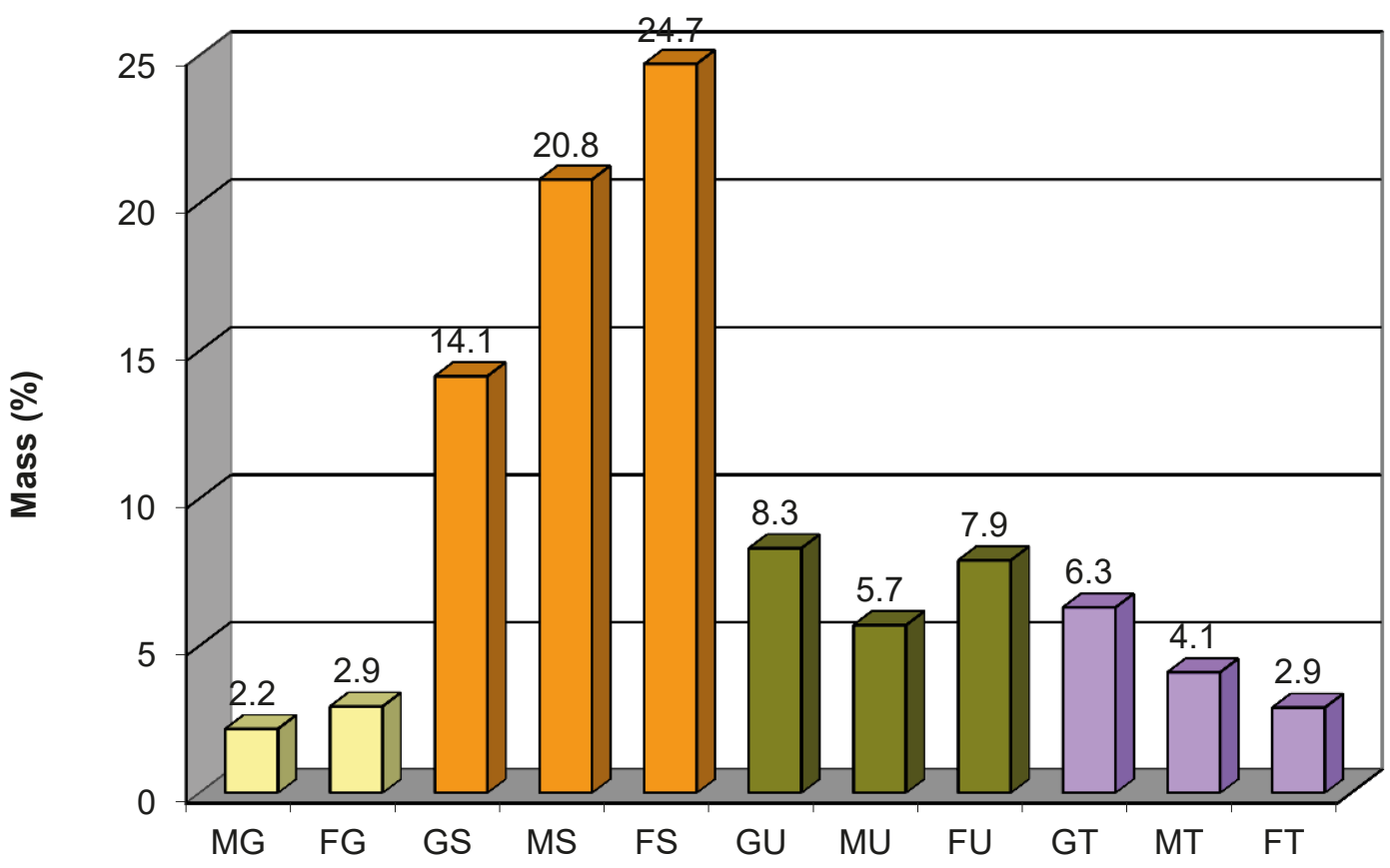

GSD 30.2 Nyarma. Sample 15407. Temple IV. Adobe brick.

Top: Cumulative sum. Bottom: Grain size classes. 


\section{7 - Nyarma. Temple IV \\ Adobe brick}

\section{Cumulative sum}

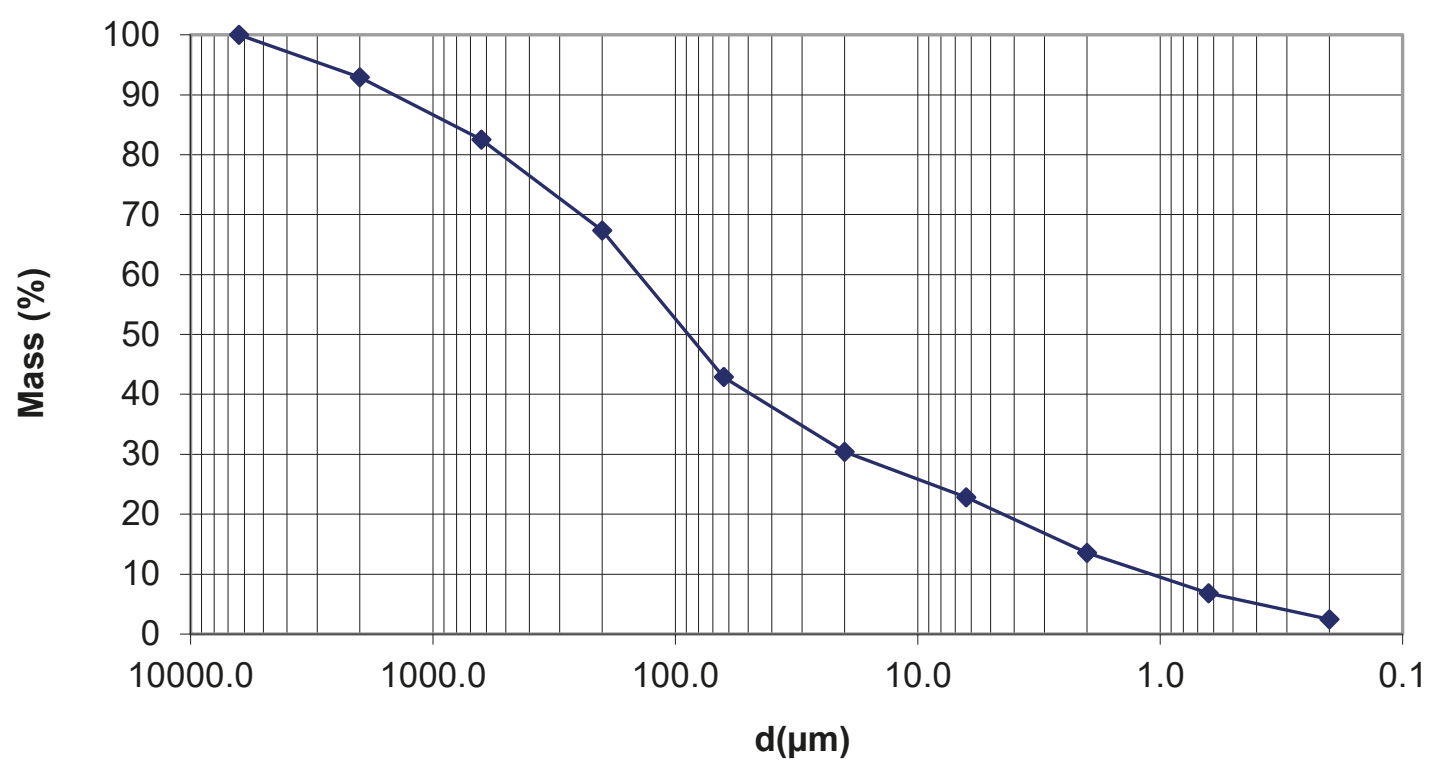

Grain size classes

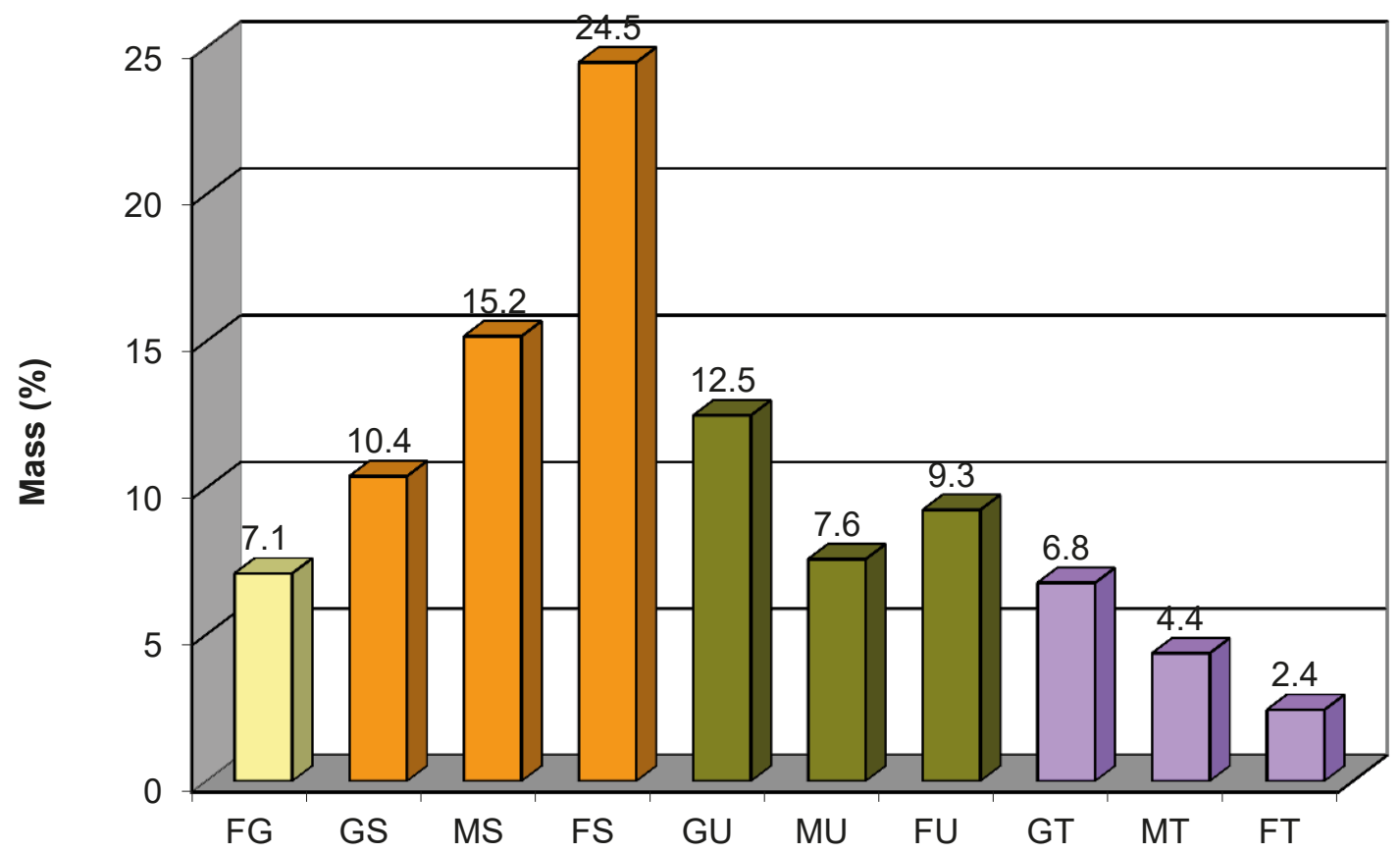

GSD 31.2 Nyarma. Sample 11917. Temple IV. Adobe brick.

Top: Cumulative sum. Bottom: Grain size classes. 


\section{5 - Nyarma. Temple Vla Adobe brick}

\section{Cumulative sum}

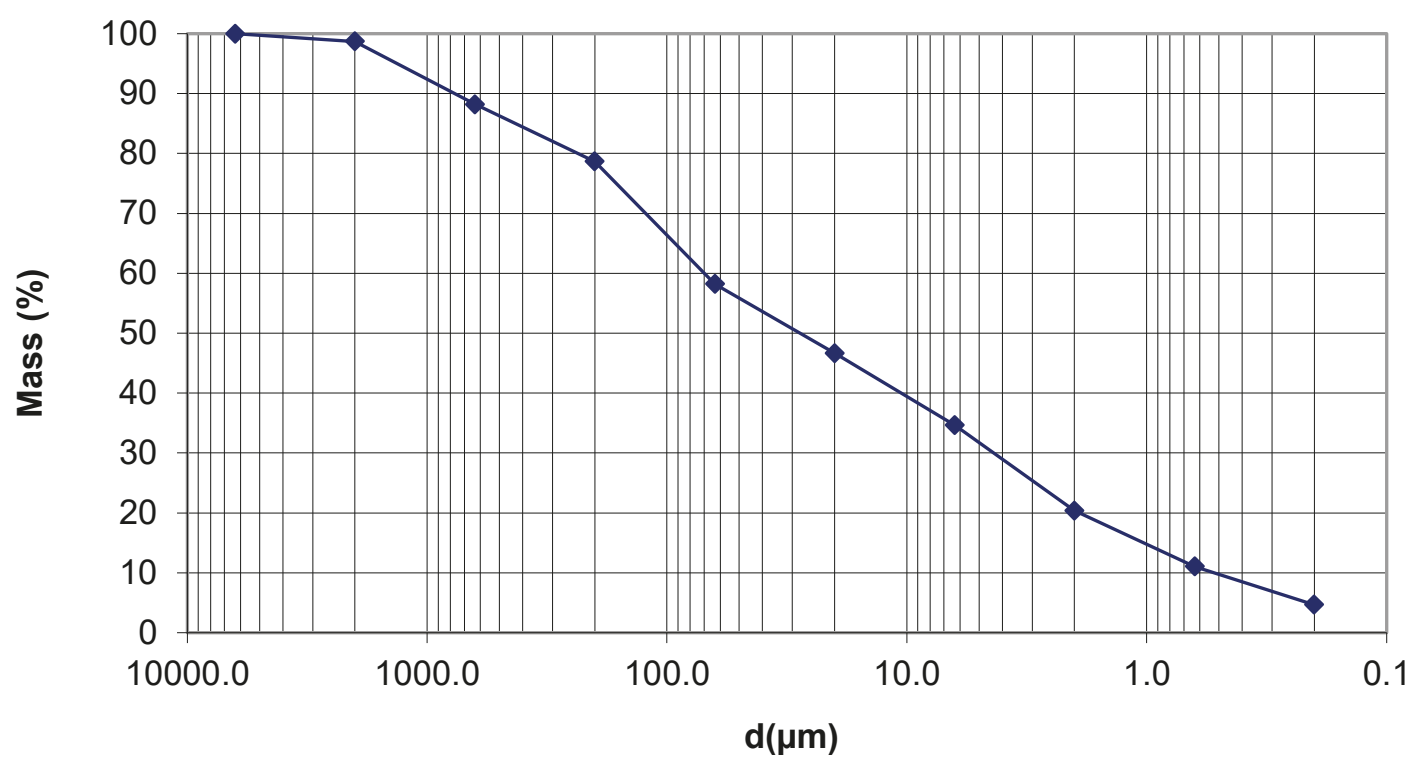

Grain size classes

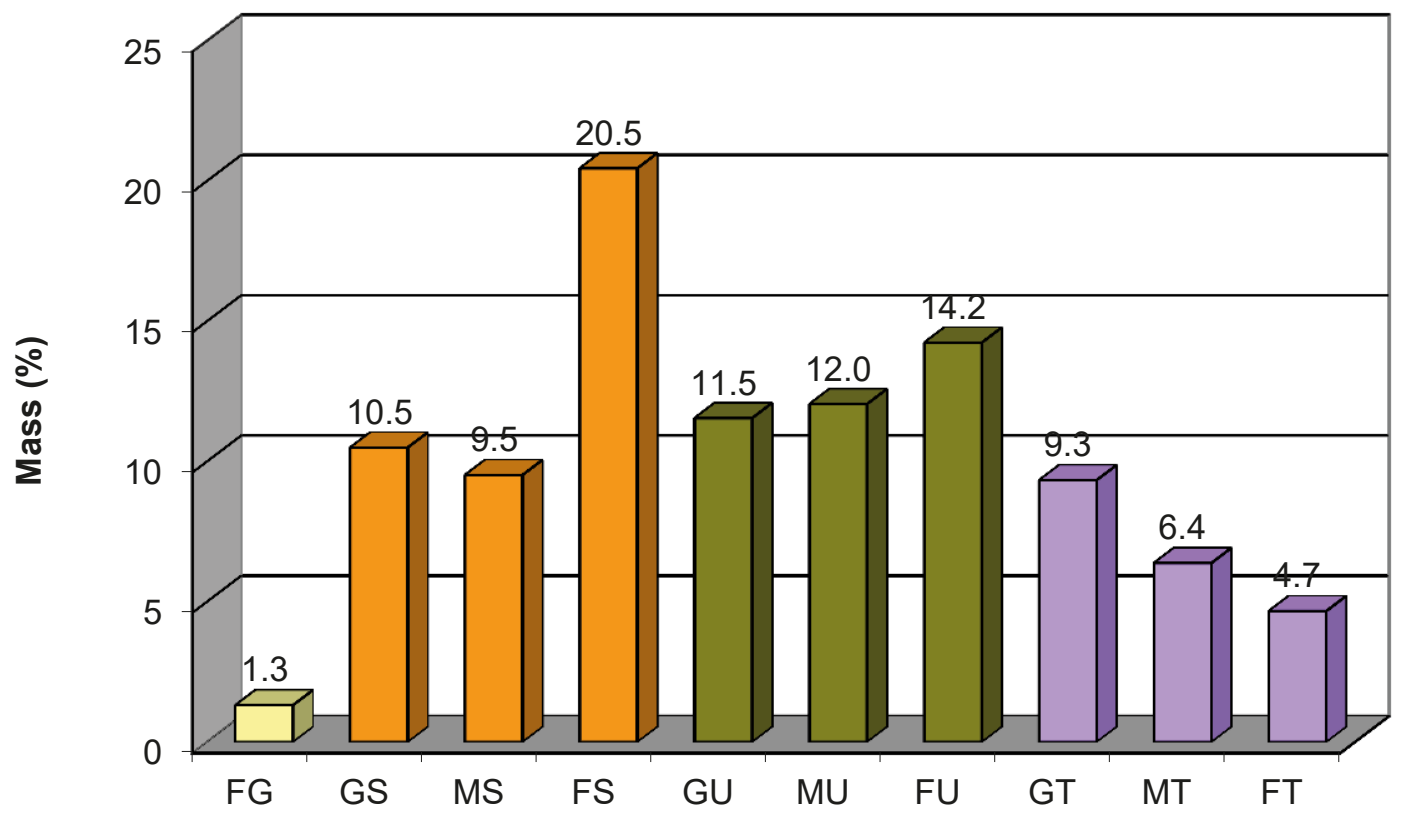

GSD 32.2 Nyarma. Sample 11765. Temple VIa. Adobe brick.

Top: Cumulative sum. Bottom: Grain size classes. 


\section{0 - Nyarma. Temple VIb Adobe brick}

\section{Cumulative sum}

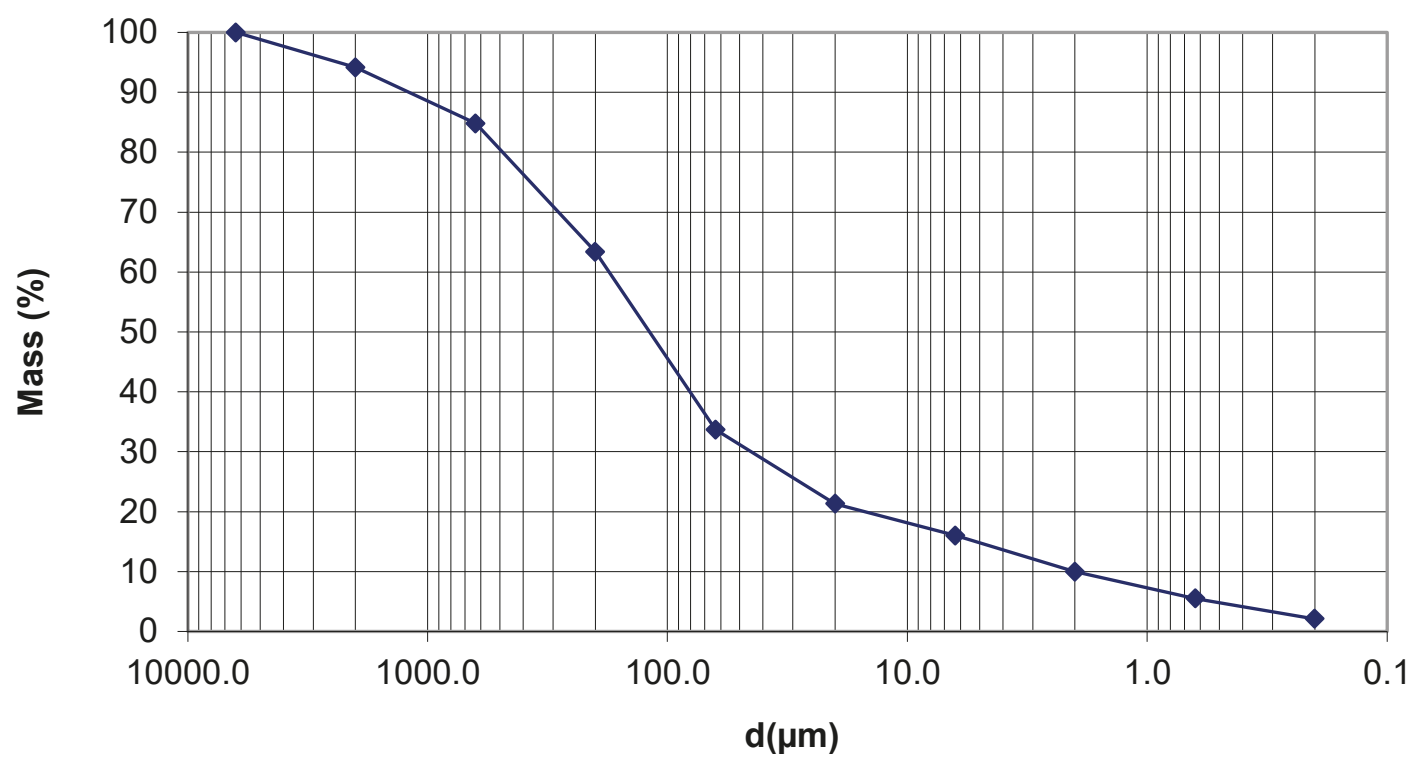

Grain size classes

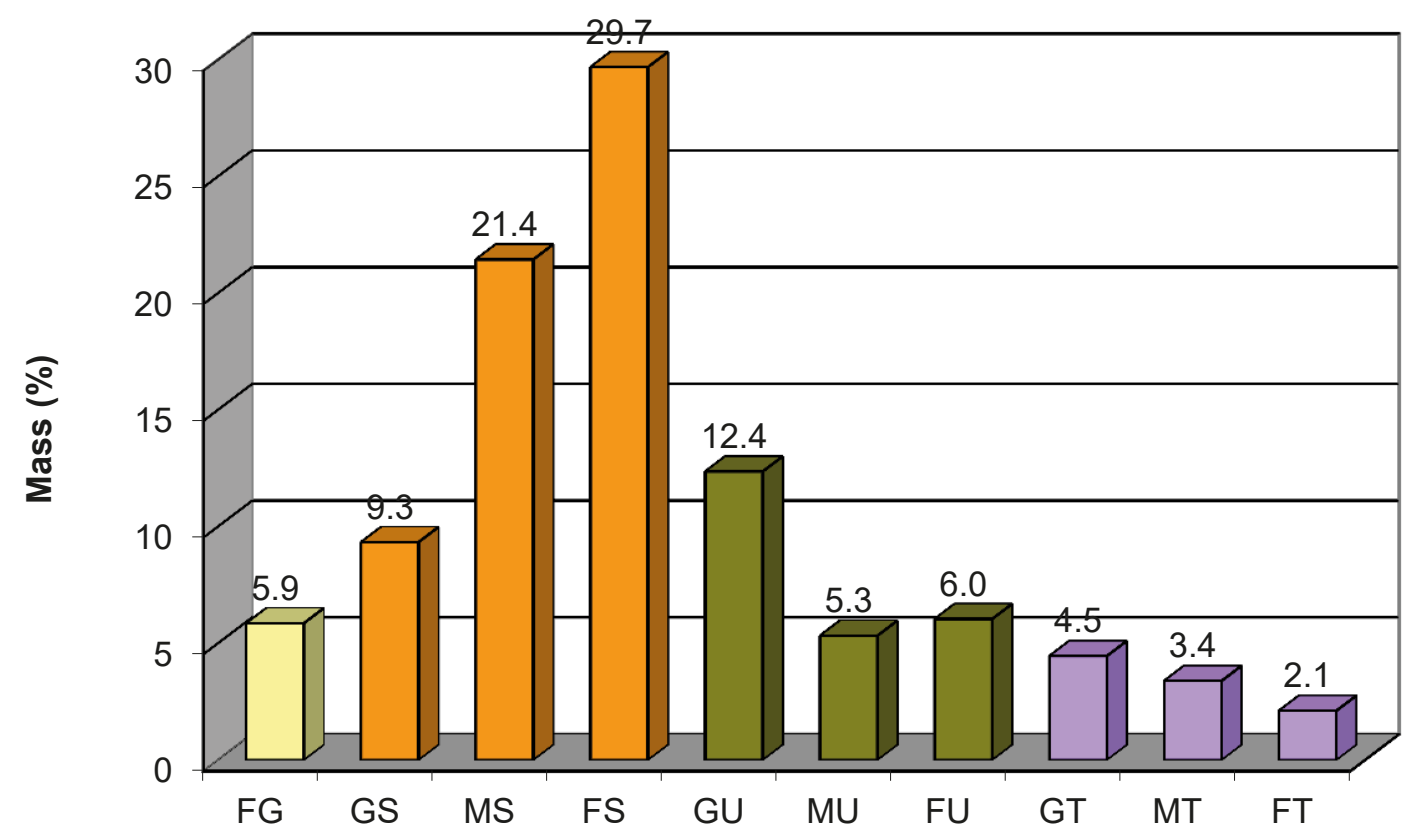

GSD 33.2 Nyarma. Sample 11760. Temple VIb. Adobe brick.

Top: Cumulative sum. Bottom: Grain size classes. 


\section{6 - Nyarma. Enclosure wall Adobe brick}

\section{Cumulative sum}

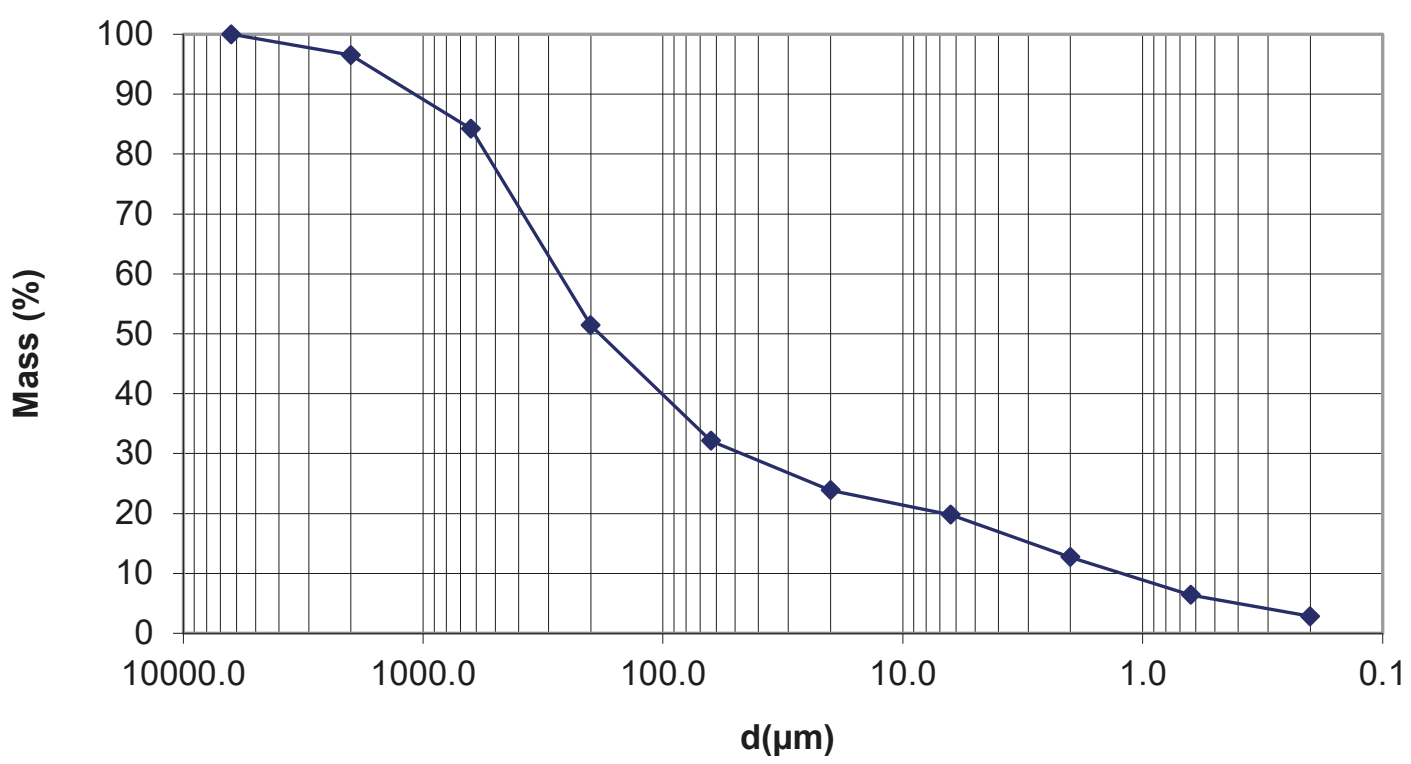

Grain size classes

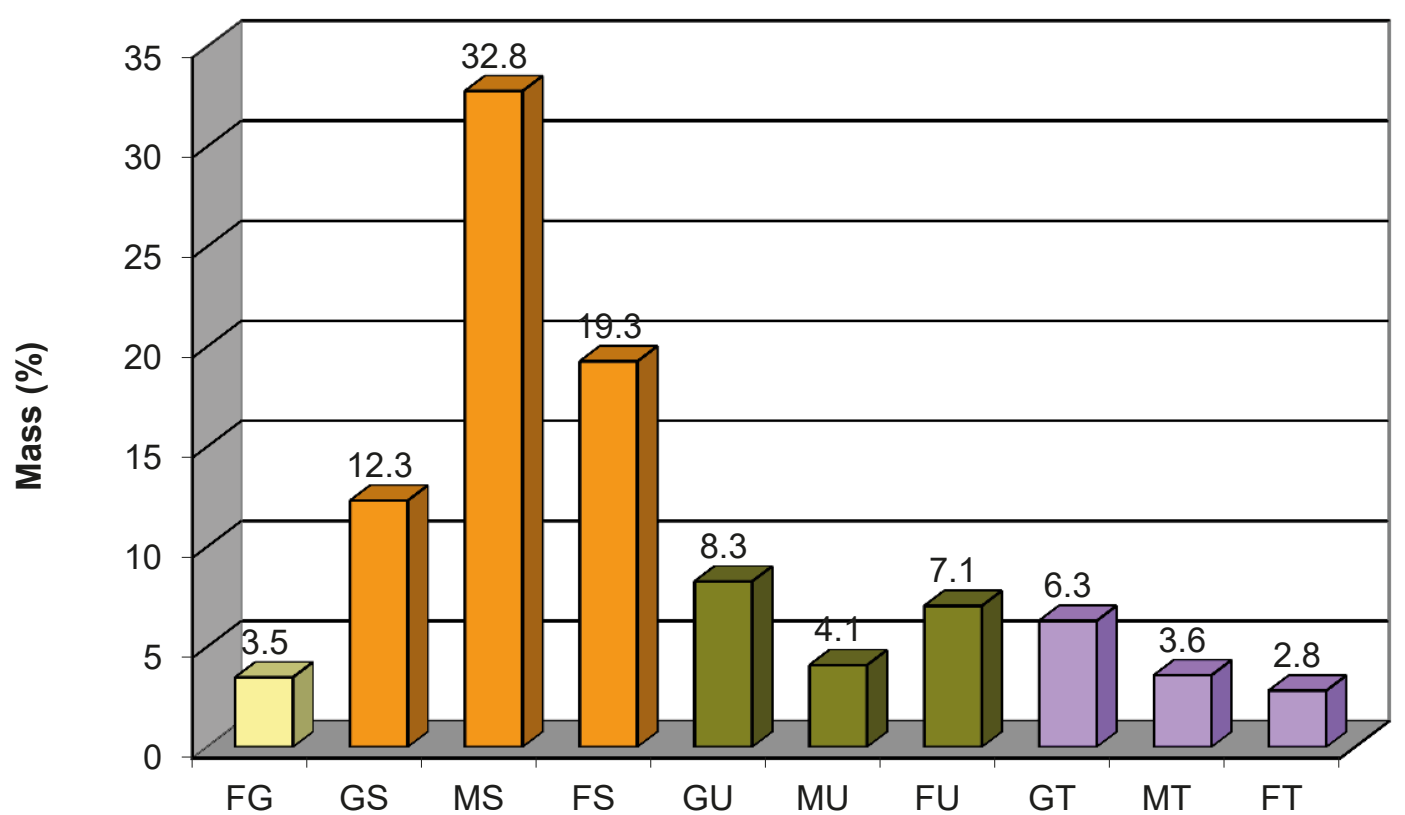

GSD 34.2 Nyarma. Sample 11766. Enclosure wall. Adobe brick.

Top: Cumulative sum. Bottom: Grain size classes. 


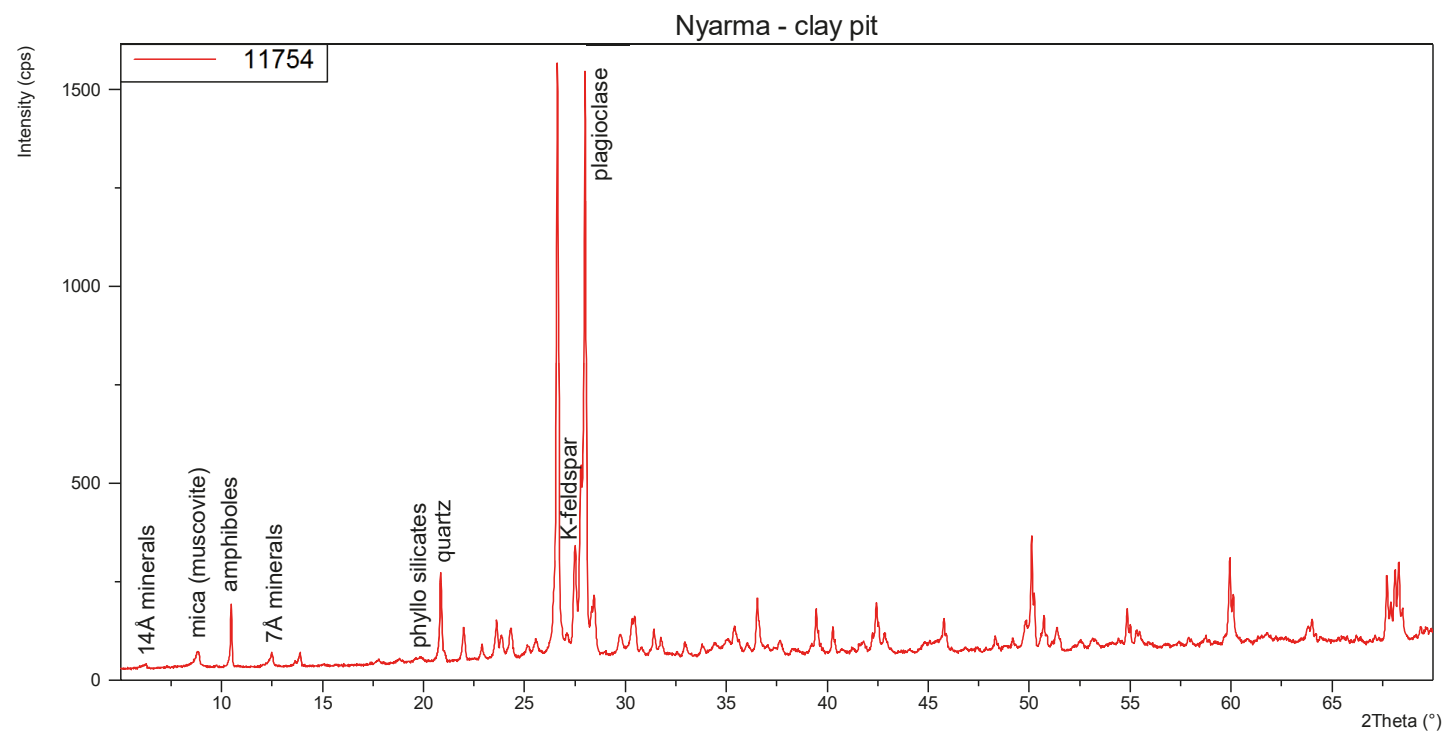

BMA 1.2 Nyarma. Sample 11754. Clay pit.

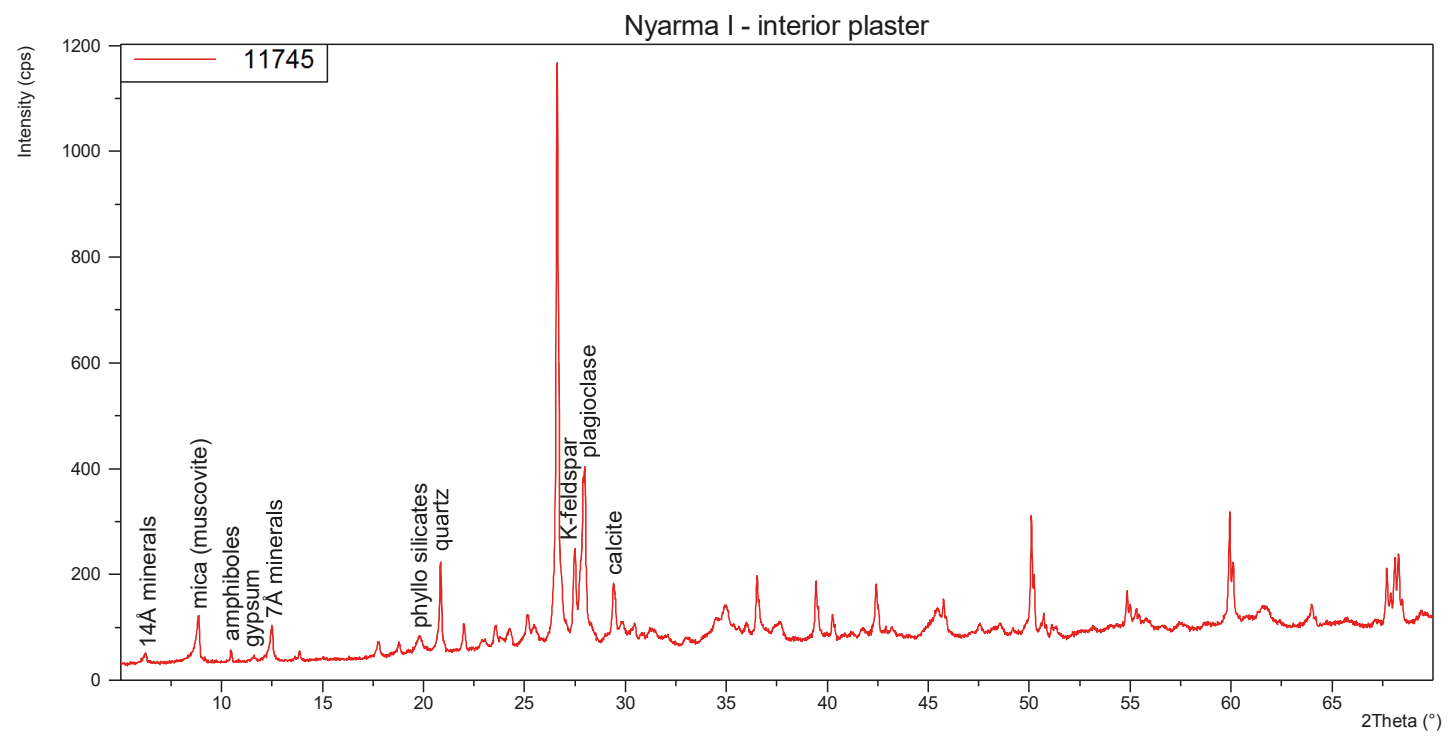

BMA 2.2 Nyarma. Sample 11745. Temple I. Interior plaster. 


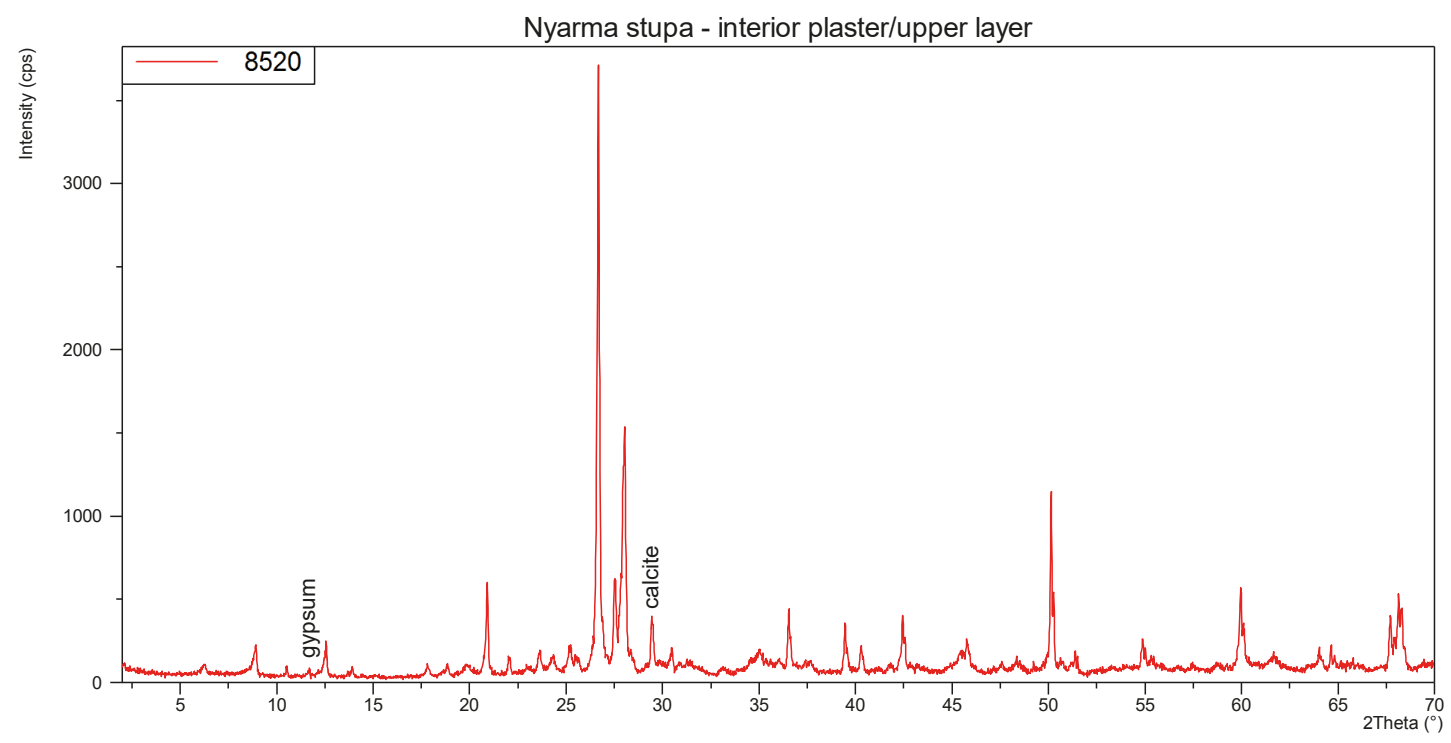

BMA 3.2 Nyarma. Stupa. Sample 8520. Interior plaster. Upper layer.

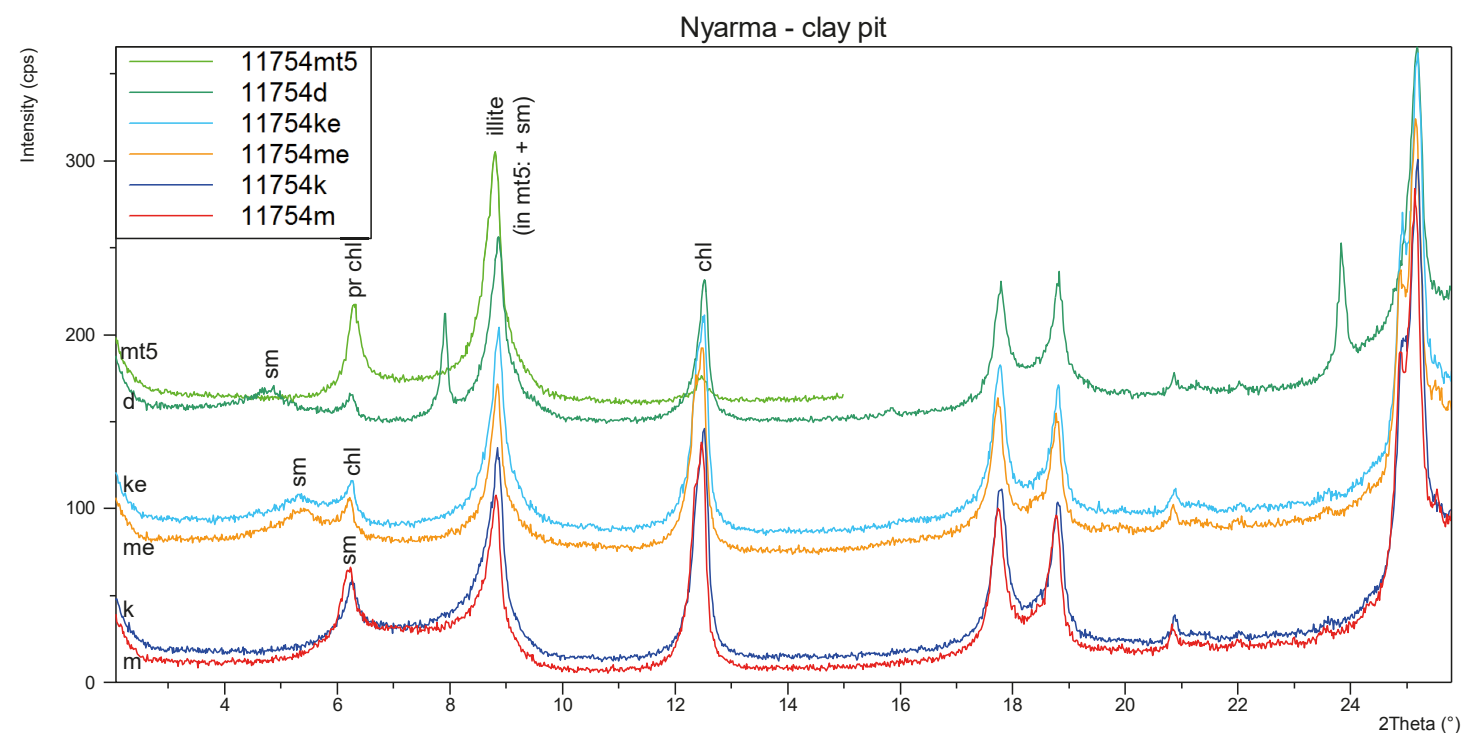

CMA 1.2 Nyarma. Sample 11754. Clay pit. 


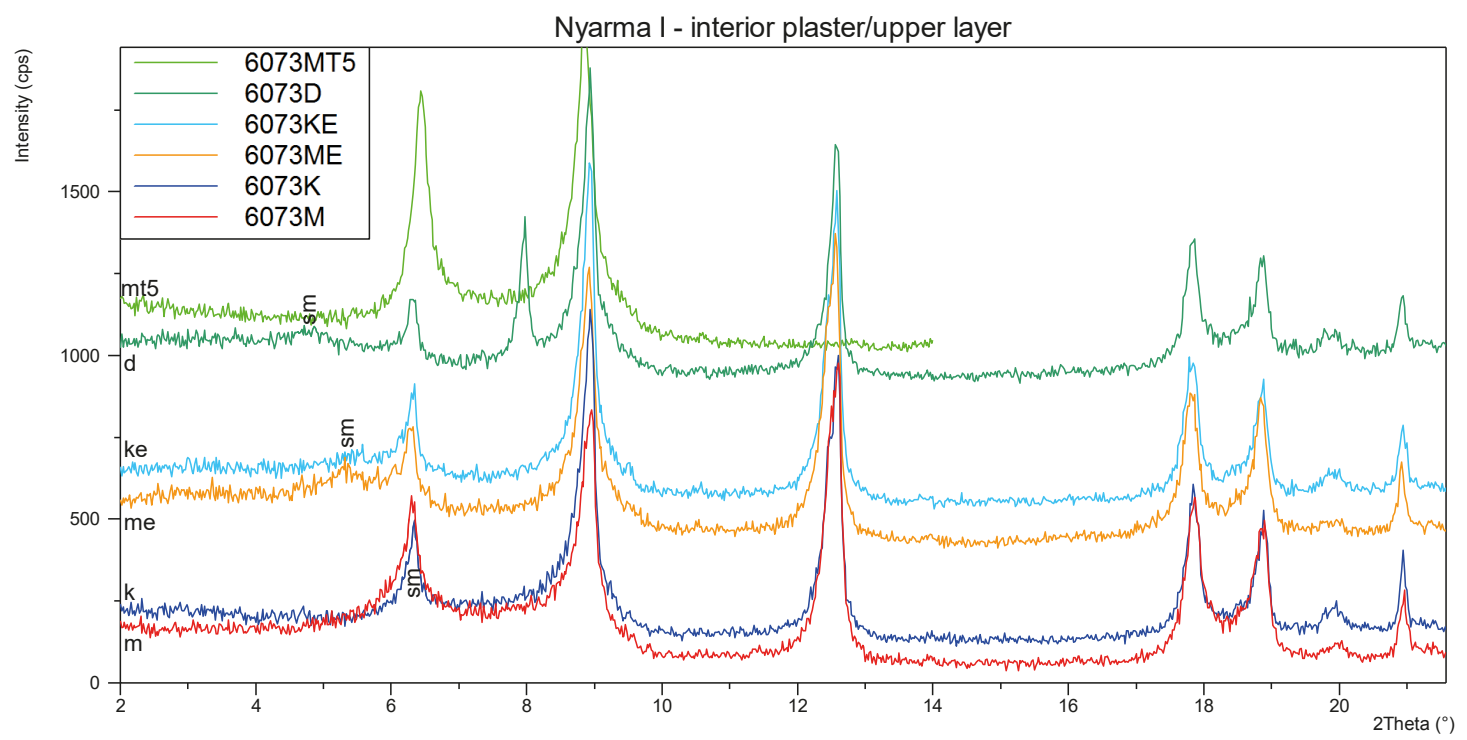

CMA 2.2 Nyarma I. Main temple. Sample 6073. Interior plaster. Upper layer.

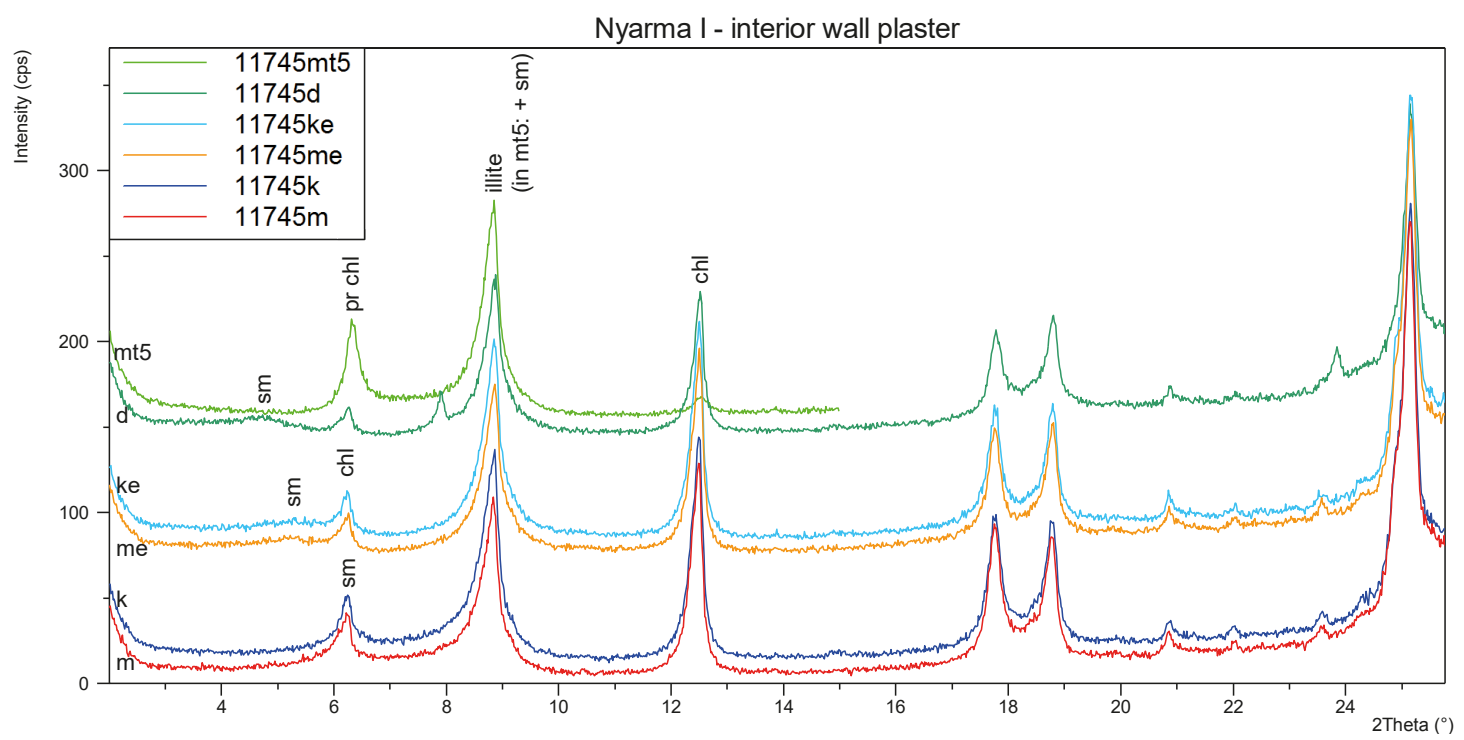

CMA 3.2 Nyarma. Sample 11745. Temple I. Interior plaster. 
Nyarma I - adobe brick

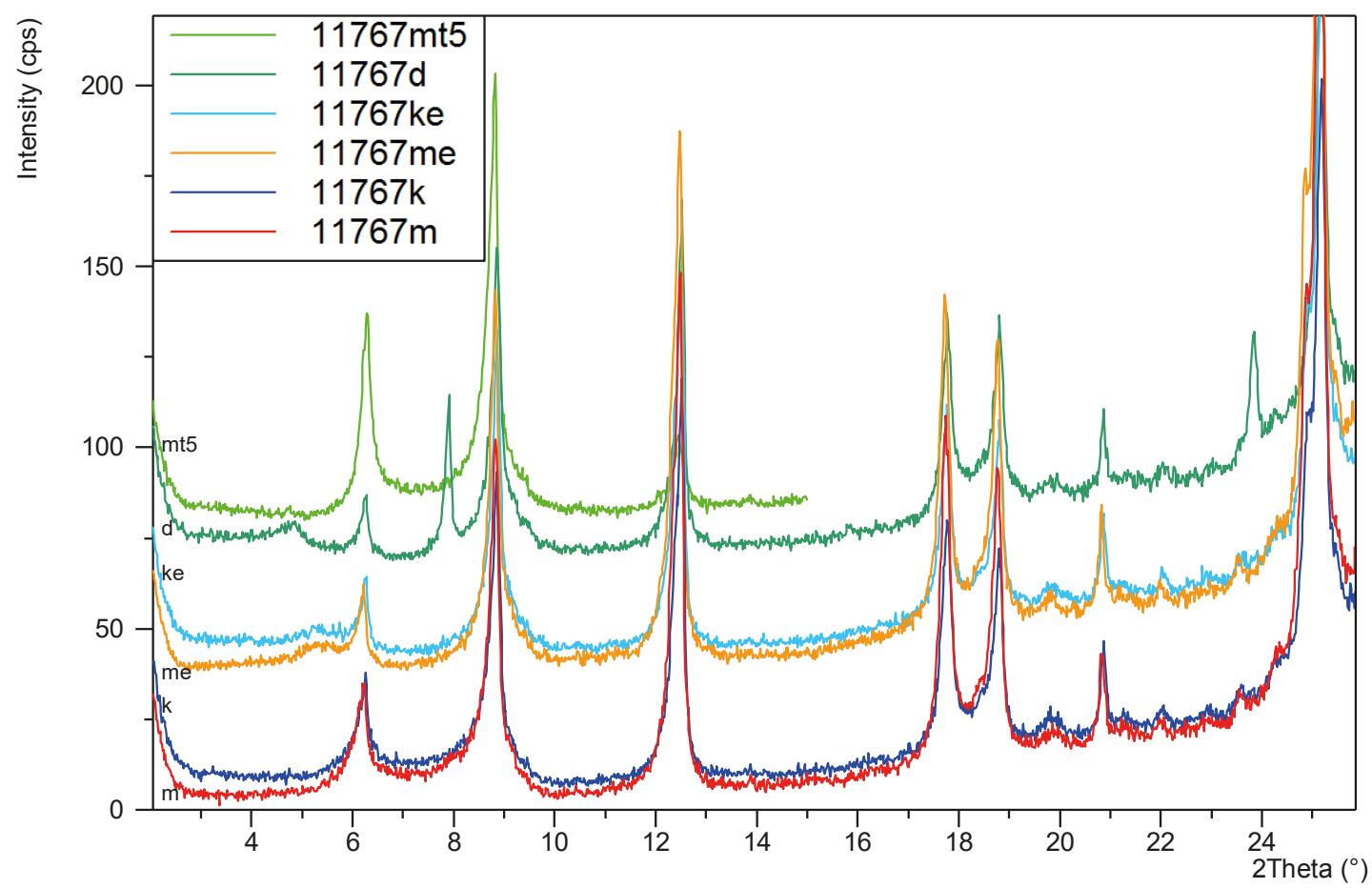

CMA 4.2 Nyarma. Sample 11767. Temple I. Adobe brick.

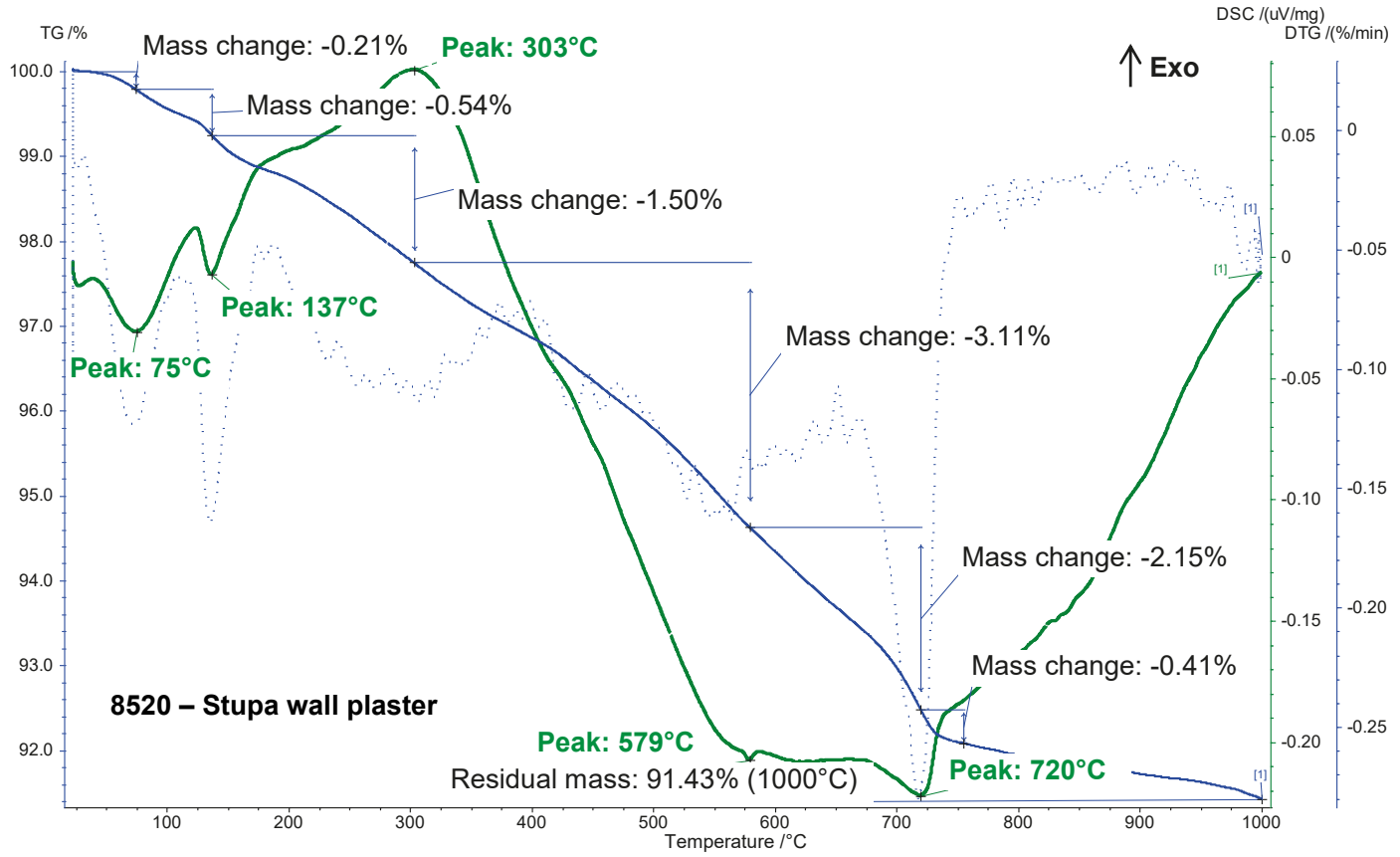

STA 1.2 Nyarma. Sample 8520. Stupa. Wall plaster. 


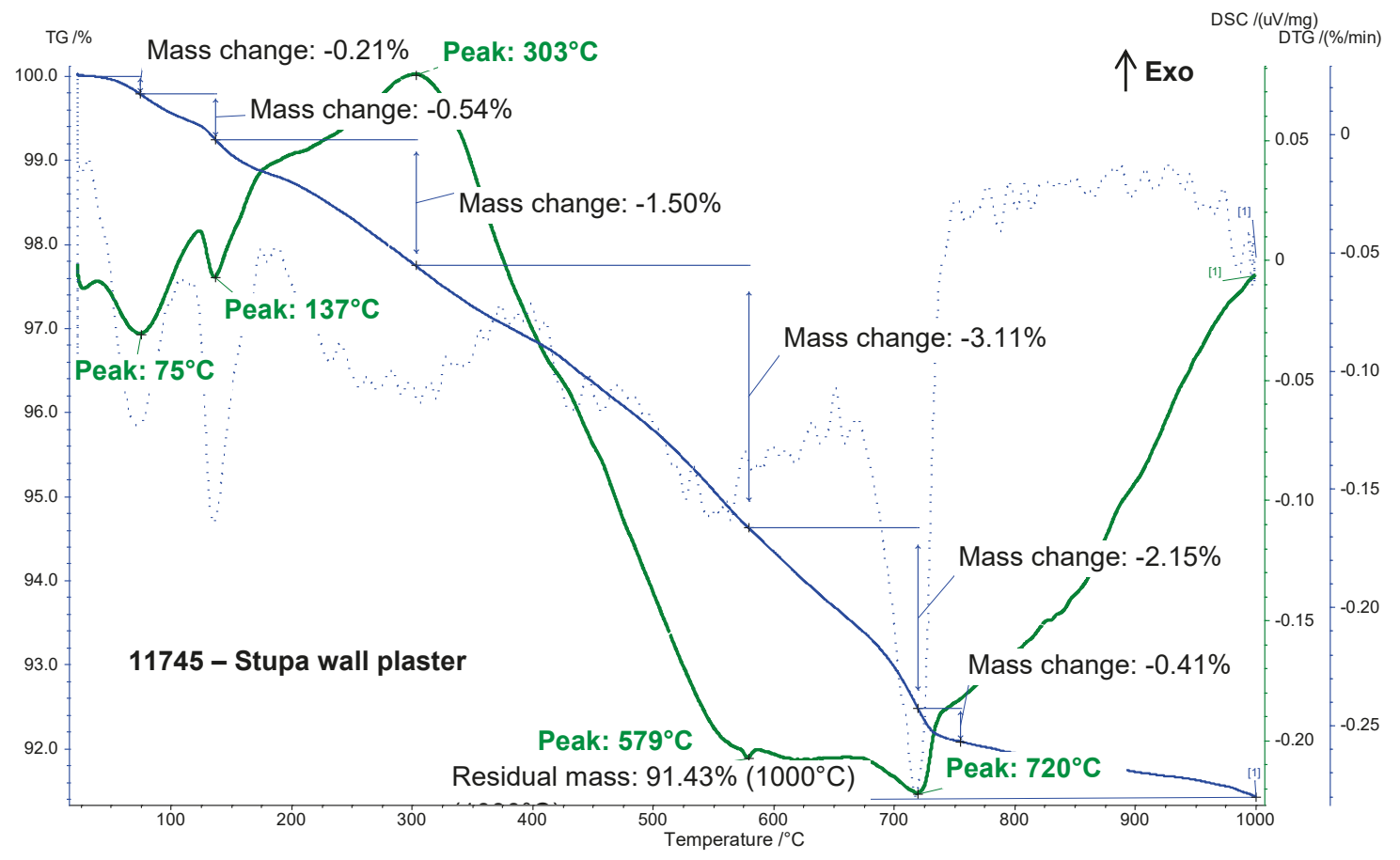

STA 2.2 Nyarma. Sample 11745. Stupa. Wall plaster. 
